\title{
Herschel and SCUBA-2 observations of dust emission in a sample of Planck cold clumps ${ }^{\star} \star \star$
}

\author{
Mika Juvela ${ }^{1}$, Jinhua $\mathrm{He}^{2,3,4,5}$, Katherine Pattle ${ }^{6}$, Tie Liu ${ }^{7,8}$, George Bendo ${ }^{9}$, David J. Eden ${ }^{10}$, Orsolya Fehér ${ }^{11,12}$, \\ Michel Fich ${ }^{13}$, Gary Fuller ${ }^{9}$, Naomi Hirano ${ }^{14}$, Kee-Tae Kim ${ }^{7}$, Di Li ${ }^{15}, 16$, Sheng-Yuan Liu ${ }^{14}$, Johanna Malinen ${ }^{17}$, \\ Douglas J. Marshall ${ }^{18}$, Deborah Paradis ${ }^{19,20}$, Harriet Parsons ${ }^{8}$, Veli-Matti Pelkonen ${ }^{1}$, Mark G. Rawlings ${ }^{8}$, \\ Isabelle Ristorcelli1 ${ }^{19,20}$, Manash R. Samal ${ }^{21}$, Ken'ichi Tatematsu ${ }^{22}$, Mark Thompson ${ }^{23}$, Alessio Traficante ${ }^{24}$, \\ Ke Wang ${ }^{25}$, Derek Ward-Thompson ${ }^{6}$, Yuefang $\mathrm{Wu}^{26}$, Hee-Weon $\mathrm{Yi}^{27}$, and Hyunju $\mathrm{Yoo}^{28}$
}

(Affiliations can be found after the references)

Received 9 September 2017 / Accepted 2 December 2017

\begin{abstract}
Context. Analysis of all-sky Planck submillimetre observations and the IRAS $100 \mu \mathrm{m}$ data has led to the detection of a population of Galactic cold clumps. The clumps can be used to study star formation and dust properties in a wide range of Galactic environments. Aims. Our aim is to measure dust spectral energy distribution (SED) variations as a function of the spatial scale and the wavelength. Methods. We examined the SEDs at large scales using IRAS, Planck, and Herschel data. At smaller scales, we compared JCMT/SCUBA-2 $850 \mu \mathrm{m}$ maps with Herschel data that were filtered using the SCUBA-2 pipeline. Clumps were extracted using the Fellwalker method, and their spectra were modelled as modified blackbody functions.

Results. According to IRAS and Planck data, most fields have dust colour temperatures $T_{\mathrm{C}} \sim 14-18 \mathrm{~K}$ and opacity spectral index values of $\beta=1.5-1.9$. The clumps and cores identified in SCUBA- 2 maps have $T \sim 13 \mathrm{~K}$ and similar $\beta$ values. There are some indications of the dust emission spectrum becoming flatter at wavelengths longer than $500 \mu \mathrm{m}$. In fits involving Planck data, the significance is limited by the uncertainty of the corrections for $\mathrm{CO}$ line contamination. The fits to the SPIRE data give a median $\beta$ value that is slightly above 1.8. In the joint SPIRE and SCUBA-2 $850 \mu \mathrm{m}$ fits, the value decreases to $\beta \sim 1$.6. Most of the observed $T-\beta$ anticorrelation can be explained by noise.

Conclusions. The typical submillimetre opacity spectral index $\beta$ of cold clumps is found to be $\sim 1.7$. This is above the values of diffuse clouds, but lower than in some previous studies of dense clumps. There is only tentative evidence of a $T-\beta$ anticorrelation and $\beta$ decreasing at millimetre wavelengths.
\end{abstract}

Key words. ISM: clouds - Infrared: ISM - Submillimetre: ISM - dust, extinction - Stars: formation - Stars: protostars

\section{Introduction}

The all-sky survey of the Planck satellite (Tauber et al. 2010), assisted by the IRAS $100 \mu \mathrm{m}$ data, made it possible to identify and to catalogue cold interstellar clouds on a Galactic scale. The angular resolution of $\sim 5^{\prime}$ is sufficient to identify compact sources that can be directly associated with the earliest stages of star formation. Analysis of the Planck and IRAS data led to the creation of the Planck Catalogue of Cold Clumps (PGCC; Planck Collaboration XXVIII 2016), which lists the properties of over 13000 sources. At distances of up to $\sim 500 \mathrm{pc}$, the Planck observations are able to resolve sub-parsec structures. For the more distant sources, up to $7 \mathrm{kpc}$, the observed signal may correspond to the detection of entire clouds. Because the PGCC detection method is based on the temperature of the sources,

\footnotetext{
* Planck (http://www.esa.int/Planck) is a project of the European Space Agency - ESA - with instruments provided by two scientific consortia funded by ESA member states (in particular the lead countries: France and Italy) with contributions from NASA (USA), and telescope reflectors provided in a collaboration between ESA and a scientific consortium led and funded by Denmark.

${ }^{\star \star}$ Herschel is an ESA space observatory with science instruments provided by European-led Principal Investigator consortia and with important participation from NASA.
}

which is relatively cold compared to their environment (Montier et al. 2010), the low temperatures (typically $T \lesssim 14 \mathrm{~K}$ ) are a proxy for high column densities and also exclude the possibility of very strong internal heating. Even when they are spatially unresolved, the Planck clumps are thus likely to contain higher density substructure, including gravitationally bound pre-stellar and proto-stellar cores.

Some PGCC objects have been studied in detail using the Herschel follow-up observations made in the Herschel key programme Galactic Cold Cores (GCC). In the GCC, 116 fields were selected from an early version of the Planck clump catalogue (Planck Collaboration XXIII 2011), and the fields were mapped with Herschel PACS and SPIRE instruments (Pilbratt et al. 2010; Poglitsch et al. 2010; Griffin et al. 2010) between 100 and $500 \mu \mathrm{m}$ (Juvela et al. 2010; Planck Collaboration XXII 2011; Juvela et al. 2012). The size of each Herschel map is $\sim 40^{\prime}$. This means that the fields typically contain a few Planck clumps, but also a significant area of the surrounding cloud and even diffuse medium. This has enabled, for example, the study of the filamentary structure of the clouds around the clumps (Rivera-Ingraham et al. 2016, 2017; Malinen et al. 2016).

To study the variations in the submillimetre dust opacity, Juvela et al. (2015b) compared the $250 \mu \mathrm{m}$ optical depths to the near-infrared (NIR) extinctions. The typical value of 
$\tau(250 \mu \mathrm{m}) / \tau(\mathrm{J})$ in the fields was found to be $1.6 \times 10^{-3}$, which is more than twice the values in diffuse medium (Planck Collaboration XI 2014), but consistent with other Planck studies of molecular clouds (Planck Collaboration Int. XIV 2014) (see also e.g. Martin et al. 2012; Roy et al. 2013; Lombardi et al. 2014). The submillimetre opacity was also found to be correlated with column density (and anticorrelated with temperature), with individual cores reaching values still higher by a factor of two.

Juvela et al. (2015a) studied the value and variations of the opacity spectral index $\beta$ in GCC fields. Based on the IRAS and Planck data, the average value was found to be $\beta=1.84$, somewhat higher than the typical Planck values for molecular clouds at larger scales (e.g. Planck Collaboration XXV 2011; Planck Collaboration Int. XIV 2014; Planck Collaboration XI 2014). The analysis of Planck data down to $217 \mathrm{GHz}$ suggested a flattening of the dust emission spectrum (cf. Planck Collaboration Int. XIV 2014). There is strong evidence of spectral index variations within the individual fields, and the cold cores were associated with submillimetre spectral index values of $\beta \sim 2.0$ or even higher. Thus, the results are qualitatively consistent with many earlier observations of the $T-\beta$ anticorrelation (Dupac et al. 2003; Désert et al. 2008; Rodón et al. 2010; Veneziani et al. 2010; Paradis et al. 2010; Etxaluze et al. 2011; Planck Collaboration XXII 2011; Planck Collaboration Int. XVII 2014; Planck Collaboration Int. XIV 2014; Planck Collaboration XVII 2011; Planck Collaboration XIX 2011). In such studies, we recall that the colour temperature and the apparent $\beta$ values are affected by both noise and temperature variations (Shetty et al. 2009b,a; Juvela \& Ysard 2012a,b; Malinen et al. 2011; Ysard et al. 2012; Juvela et al. 2013; Pagani et al. 2015). In particular, to estimate the effect of noise on the apparent $T-\beta$ anticorrelation, we need very precise knowledge of all error sources.

The above studies were limited to wavelengths below $500 \mu \mathrm{m}$ or did include longer wavelengths but at a much lower resolution. The dust spectrum of cores can be further constrained by ground-based millimetre wavelength observations. However, for spectral index values, the results have shown a very wide range of $\beta$ estimates, from $\sim 1$ to values far above 2.0 (Shirley et al. 2005; Friesen et al. 2005; Schnee et al. 2010; Sadavoy et al. 2013, 2016; Ward-Thompson et al. 2016; Chen et al. 2016). The large scatter is partly explained by the different wavelengths used, the spatial filtering resulting from sky noise reduction (when ground-based and space-borne observations are combined), and the varying nature of the sources (e.g. low values in regions with large temperature variations). Because of the additional problems in correlating the changes of the apparent dust spectra with the intrinsic dust properties, it is safe to say that our understanding of dust evolution across the star formation process is still very incomplete. This has implications for the estimates of the mass and the detailed structure of star-forming clouds. Accurate estimates of dust properties could also be related to laboratory measurements, to determine how dust properties vary according to the environment (Boudet et al. 2005; Coupeaud et al. 2011; Demyk et al. 2017).

In late 2015, we started the legacy survey SCOPE (SCUBA-2 Continuum Observations of Pre-protostellar Evolution) to this end, to map about 1000 PGCCs in $850 \mu \mathrm{m}$ continuum emission with the SCUBA-2 instrument at the James Clerk Maxwell Telescope (JCMT; Liu et al. 2018, Eden et al. in prep.). Thousands of dense cores have been identified by the SCOPE survey, and most of them are either starless cores or proto-stellar cores with very young (Class 0/I) objects (see Liu et al. 2016; Tatematsu et al. 2017; Kim et al. 2017). These SCOPE sources are prime candidates for probing how pre-stellar/proto-stellar cores form and evolve, and for studying the very early stages of star formation across a wide variety of galactic environments. In addition, the $850 \mu \mathrm{m}$ data are important in providing high-resolution information of the dust emission at the scales of individual cores and at a wavelength that according to previous studies lies in the regime between the steeper far-infrared part and the flatter millimetre part of the dust emission spectrum (Paradis et al. 2012).

In this paper we examine dust properties in a set of some 90 fields associated with PGCC sources. The fields were mapped at $850 \mu \mathrm{m}$ with the SCUBA-2 instrument as part of the SCOPE pilot programme. The present sample contains the targets of the pilot programme for which Herschel photometric observations were available. The goal of the paper is to examine dust SED variations as a function of the spatial scale and as a function of the wavelength.

The structure of the paper is the following. The observations and data reduction are described in Sect. 2 and the analysis methods are explained in Sect. 3. The main results are presented in Sect. 4. We discuss the results in Sect. 5 before listing the main conclusions in Sect. 6.

\section{Observational data}

\subsection{SCUBA-2 observations}

The target selection for SCUBA-2 observations was based on the PGCC catalogue, but also employed Herschel follow-up observations to pinpoint the exact locations of the column density maxima. Thus, all SCUBA-2 maps are not centred on PGCC coordinates, and one of the fields, G150.4+3.9A2, is not intersected by any PGCC clumps. The fields are listed in Table H.1.

The SCUBA-2 (Holland et al. 2013) maps at $850 \mu \mathrm{m}$ were observed at JCMT between April and October 2015 (projects M15AI05 and M15BI061, PI: Tie Liu). The spatial resolution of the data is $14^{\prime \prime}$. All observations employed the CV Daisy mode (Bintley et al. 2014), resulting in maps with an approximate diameter of $12^{\prime}$ and a roughly constant integration time within the innermost $3^{\prime}$. The observations of all fields were of equal length with a total observing time of some 20 minutes per field and a median exposure time of some 80 seconds per map position. The observing conditions typically corresponded to $\tau(225 \mathrm{GHz})=0.10-0.15$. Maps with a $4.0^{\prime \prime}$ pixel size were created using the makemap task of the Starlink SMURF package (Jenness et al. 2011; Chapin et al. 2013). The final noise values are very similar in all fields. The approximate rms noise values are $5.6 \mathrm{mJy}$ beam $^{-1}$ for the entire maps and $3.4 \mathrm{mJy} \mathrm{beam}^{-1}$ for the central area within a $5^{\prime}$ radius.

In the map making, we used filtering scales of $\theta_{\mathrm{F}}=200^{\prime \prime}$ and $\theta_{\mathrm{F}}=500^{\prime \prime}$. SCUBA-2 data are typically reduced using external masking (or an auto-masking method) where source masks are derived from the SCUBA-2 data (Sadavoy et al. 2013; Moore et al. 2015; Pattle et al. 2015). However, we relied mainly on masks derived from Herschel maps, which provide a more complete picture of the cloud structure at lower column densities. The Herschel $500 \mu \mathrm{m}$ maps were high-pass filtered with a Gaussian filter with an FWHM equal to $300^{\prime \prime}$, and the remaining emission was initially thresholded at $50 \mathrm{MJy} \mathrm{sr}^{-1}$, which corresponds approximately to the $1 \sigma$ noise of the SCUBA-2 maps (for typical values of dust temperature and spectral index). The resulting masks are thus significantly more extended than in auto-masking, where the threshold would be several times 
higher. When necessary, the mask was further adjusted so that it covered 10-30\% of the map area, and each distinct masked area was at least 40" in size (larger than the Herschel beam). Alternative masks were created the traditional way, by thresholding preliminary $850 \mu \mathrm{m}$ signal-to-noise ratio maps at a level of five. The masks were expanded by $15^{\prime \prime}$ because they were also used when Herschel data (with larger beam sizes) were processed through the SCUBA-2 pipeline, in order to match the spatial filtering that affects SCUBA-2 maps (Pattle et al. 2015). To check how the results depend on the mask size, we used a second set of Herschel-based source masks that were $50 \%$ smaller. In the following, these large and small Herschel masks are called LM and SM, respectively. The masks created using SCUBA-2 data are referred to as M850. The masks are shown in Fig. C.1.

In the following, we assume for SCUBA-2 a $10 \%$ relative uncertainty. This covers the uncertainty of the calibration and the uncertainty of the contamination by $\mathrm{CO}(3-2)$ line emission. Although the $\mathrm{CO}$ contribution in the $850 \mu \mathrm{m}$ measurements can sometimes reach some tens of percent (Drabek et al. 2012), it is usually below $10 \%$ (e.g. Moore et al. 2015; Mairs et al. 2016) and can be expected to be lower for cold clumps. The uncertainties caused by $\mathrm{CO}$ emission are discussed in Appendix B.

\subsection{Herschel observations}

The Herschel SPIRE data at $250 \mu \mathrm{m}, 350 \mu \mathrm{m}$, and $500 \mu \mathrm{m}$ were taken from the Herschel Science Archive (HSA) ${ }^{1}$. We used the level 2.5 maps produced by the standard data reduction pipelines and calibrated for extended emission (the so-called photometer extended map product). The quality of the SPIRE maps in HSA is good, and we find no need for manual data reduction. Out of the 96 fields, 43 were observed as part of the GCC project (Juvela et al. 2010). For consistency, we used HSA pipeline data for these fields as well.

The resolutions of the SPIRE observations are 18.4", 25.2", and $36.7^{\prime \prime}$ for the $250 \mu \mathrm{m}, 350 \mu \mathrm{m}$, and $500 \mu \mathrm{m}$ bands, respectively $^{2}$. The maps were convolved to $40^{\prime \prime}$ and fitted with modified blackbody (MBB) curves with $\beta=1.8$. These initial SED estimates were used to colour correct the SPIRE maps to obtain monochromatic values at the nominal wavelengths. The SCUBA-2 data were later colour corrected using the same SEDs. In the temperature range of $T=10-20 \mathrm{~K}$, the corrections are $0-2 \%$ for both SPIRE and SCUBA-2. The effect on the SED shapes and especially on the $\beta$ estimates are even smaller because the corrections are correlated between the bands (see Appendix A). We adopted a relative uncertainty of $4 \%$ for the SPIRE bands, with a $\rho=0.5$ correlation between the bands (Bendo et al. 2013). The assumed correlation between the photometric values decreases the formal $T$ and $\beta$ uncertainty in the fits to SPIRE data (Galametz et al. 2012). However, when SPIRE is combined with SCUBA-2, the situation may no longer be true. If the $250-500 \mu \mathrm{m}$ data have errors preferentially in the same direction, the effect on the SED shape is large when SPIRE is combined with the (presumably) uncorrelated $850 \mu \mathrm{m}$ data point.

The main analysis was made without PACS data, which were only compared to the SED fits of longer wavelength measurements. The reason is that PACS observations cover only a small fraction of the SCUBA-2 fields and including shorter wavelengths can bias estimates of the spectral index, especially in the

\footnotetext{
1 http://archives.esac.esa.int/hsa/whsa/

2 The Spectral and Photometric Imaging Receiver (SPIRE) Handbook, http://herschel. esac.esa.int/Docs/SPIRE/

spire_handbook.pdf
}

Table 1. PACS observations.

\begin{tabular}{ll}
\hline \hline Field & PACS observation numbers \\
\hline G070.4-01.5A1 & 1342220091,1342220092 \\
G111.6+20.2A1 & 1342198861,1342198862 \\
G113.4+16.9A1 & 1342197673,1342197674 \\
G115.9+09.4A1 & 1342220665,1342220666 \\
G127.67+2.65 & 1342216511 \\
G127.67+2.65 & 1342216512 \\
G130.3+11.2A1 & 1342218718,1342218719 \\
G131.7+09.7A1 & 1342218640 \\
G131.7+09.7A1 & 1342223882 \\
G132.0+08.9A1 & 1342223880,1342223881 \\
G144.8+00.7A1 & 1342226995,1342226996 \\
G149.60+3.45 & 1342217532,1342217533 \\
G150.4+03.9A2 & 1342217530,1342217531 \\
G151.4+03.9A1 & 1342205042 \\
G151.4+03.9A1 & 1342205043 \\
G154.0+05.0A1 & 1342217528,1342217529 \\
G157.12-8.72 & 1342216415 \\
G157.12-8.72 & 1342216416 \\
G157.93-2.51 & 1342217526,1342217527 \\
G159.0-08.4A1 & 1342239276,1342239277 \\
G159.21-34.28 & 1342239263,1342239264 \\
G160.6-16.7A1 & 1342216420,1342216421 \\
G162.4-08.7A1 & 1342239278,1342239279 \\
G163.82-8.33 & 1342205047,1342205048 \\
G171.14-17.57 & 1342204860,1342204861 \\
G172.8-14.7A1 & 1342228001,1342228002, \\
G173.9-13.7A1 & 1342228003,1342228004 \\
G174.0-15.8A1 & 1342228005,1342228006 \\
G177.6-20.3A1 & 1342202250,1342202251 \\
G201.2+00.4A1 & 1342269260,1342269261, \\
G202.00+2.65 & 1342269262,1342269263 \\
G204.8-13.8A1 & 1342228371,1342228372 \\
G210.90-36.55 & 1342225212,1342225213 \\
G215.44-16.38 & 1342204305 \\
G215.44-16.38 & 1342204306 \\
G216.76-2.58 & 1342219406,1342219407 \\
G219.13-9.72 & 1342219402,1342219403 \\
\hline
\end{tabular}

presence of embedded radiation sources or emission from very small grains (e.g. Shetty et al. 2009b; Malinen et al. 2011; Juvela \& Ysard 2012b). Of the 96 fields, 37 are fully or partially covered by PACS observations (for observation numbers, see Table 1). We used the UNIMAP versions of the $160 \mu \mathrm{m}$ maps provided in the Herschel science archive (Piazzo et al. 2015). We assumed a relative uncertainty of $10 \%$ for PACS to cover the $5 \%$ calibration uncertainty (estimated for point sources) (Balog et al. 2014) and the map-making uncertainty.

\subsection{Planck and IRAS data}

We used IRAS and Planck observations to examine the dust emission at large $\sim 10^{\prime}$ scales. We used the IRIS version of the $100 \mu \mathrm{m}$ IRAS data (Miville-Deschênes \& Lagache 2005) and the $857 \mathrm{GHz}, 545 \mathrm{GHz}, 353 \mathrm{GHz}$, and $217 \mathrm{GHz}$ Planck maps taken from the Planck Legacy Archive $^{3}$ that correspond to

\footnotetext{
3 https://www.cosmos.esa.int/web/planck/pla
} 
the 2015 data release (Planck Collaboration I 2016) where the cosmic microwave background (CMB) emission has been subtracted. We subtracted the estimated levels of the cosmic infrared background (CIB) from Planck maps. Because the targets have high column densities, the CIB correction $\left(\sim 1 \mathrm{MJy} \mathrm{sr}^{-1}\right.$ or less in all bands) is relatively unimportant (Planck Collaboration VIII 2016). The amount of emission from stochastically heated very small grains (VSG) at $100 \mu \mathrm{m}$ is unknown. However, it could amount to some tens of percent in cold clouds ( $\mathrm{Li} \&$ Draine 2001; Compiègne et al. 2011). We did not correct for the VSG emission. Thus, the combined SED of IRAS and Planck data is expected to correspond to a higher colour temperature (and slightly altered and probably lower spectral index values) than the SED of the large grains alone (Juvela et al. 2015a).

Planck $353 \mathrm{GHz}$ and $217 \mathrm{GHz}$ bands are contaminated by CO $J=2-1$ and $J=3-2$ line emission, which is a significant source of uncertainty in the estimates of the longwavelength dust emission spectrum. The effect is more significant at $217 \mathrm{GHz}$ because $J=2-1$ is usually the stronger of the two CO lines, while dust emission at $217 \mathrm{GHz}$ is only about one fifth of the emission at $353 \mathrm{GHz}$ (assuming $\beta=1.7$ ). We corrected these two bands in the same way as in Juvela et al. (2015a). The correction made use of the Type $3 \mathrm{CO}$ maps provided by the Planck Consortium and was based on the assumption of line ratios $T_{\mathrm{A}}(2-1) / T_{\mathrm{A}}(1-0)=0.5$ and $T_{\mathrm{A}}(3-2) / T_{\mathrm{A}}(1-0)=0.3$. The justification and uncertainty of these values is discussed in Juvela et al. (2015a).

The $217 \mathrm{GHz}$ band could have additional contribution from free-free emission in the case of actively star-forming clumps. Therefore, similar to Juvela et al. (2015a), we subtracted a model for the low-frequency foregrounds ${ }^{4}$, although this is in practice much smaller than the $\mathrm{CO}$ correction.

We assumed absolute calibration uncertainties of $10 \%$ for IRAS $100 \mu \mathrm{m}, 5 \%$ for the $857 \mathrm{GHz}$ and $545 \mathrm{GHz}$ Planck channels, and $2 \%$ for the $353 \mathrm{GHz}$ and $217 \mathrm{GHz}$ channels. Because of the common calibration, we furthermore assumed a correlation of $\rho=0.5$ between the first two Planck bands and again between the last two bands. In the $353 \mathrm{GHz}$ and $217 \mathrm{GHz}$ bands, we included an additional uncertainty (added in squares) that corresponds to $30 \%$ of the applied CO correction.

\subsection{Cloud distances}

A distance estimate has been published for 86 of the 96 sources in the PGCC catalogue (Planck Collaboration XXVIII 2016). These are mostly based on 3D extinction mapping, the comparison between the observed stellar reddening, and the predictions derived from a model of the Galactic stellar distribution (Marshall et al. 2006, 2009). There is also considerable overlap between the current source sample and the fields observed as part of the Herschel key programme Galactic Cold Cores (GCC). The distances of the GCC fields were discussed in detail by Montillaud et al. (2015). With only one exception, these estimates agreed with the original PGCC values. The distance estimates are listed in Table H.2, which also notes some nearby molecular clouds and their distance estimates, if available in the SIMBAD database ${ }^{5}$ or in the cloud compilation of Dutra \& Bica (2002). The distance estimates typically have uncertainties of several tens of percent and therefore are

\footnotetext{
4 See https://wiki.cosmos.esa.int/planckpla/index.php/ CMB_and_astrophysical_component_maps

5 http://simbad.u-strasbg.fr/simbad/
}

a major source of uncertainty when the masses of the sources are estimated.

We revisited the extinction-based distance estimates using the method MACHETE (Marshall, in prep.) The method is based on the observed reddening of background stars, which is compared to predictions based on the Besançon model (Robin et al. 2003) of stellar distributions and the optimised model of the dust distribution along the line of sight. Apart from the improvements in the method itself, we also used Herschel data to better separate the high-column-density regions associated with the SCUBA-2 targets. In practice, we used masks that covered an area where $\tau(250 \mu \mathrm{m})$ is above its $40 \%$ percentile value (separately within each map). The masks are typically larger than the size of the individual PGCC clumps and thus should provide better statistics for the background stars.

\section{Methods}

\subsection{Clump extraction}

Clumps were extracted from SCUBA-2 $850 \mu \mathrm{m}$ signal-to-noise ratio maps with the Fellwalker method (Berry 2015), which is also used by the SCOPE project (Liu et al. 2018). Clumps were required to contain more than 10 pixels and to rise above three times the rms value $\sigma$. The other relevant parameters of the algorithm were $M I N D I P=2 \sigma$ and $M A X J U M P=5$ pixels $^{6}$. The clump extraction was repeated for each version of the reduced data (e.g. the three different source masks, LM, SM, and M850). The extraction can result in spurious detections near the noisy map boundaries, but these are rejected later when the analysis is limited to structures within $5^{\prime}$ of the centre of each field.

\subsection{Clump photometry}

Clump fluxes were measured from the original Herschel maps and from SCUBA-2 $850 \mu \mathrm{m}$ and Herschel maps processed through the SCUBA-2 pipeline. To make the procedure consistent across maps of originally different spatial resolution, the photometric measurements were made on maps that were first all convolved to a common resolution of $40^{\prime \prime}$.

The measurement apertures were based on the footprints of the Fellwalker clumps, but were extended by $20^{\prime \prime}$ to reduce the flux losses resulting from the above-mentioned map convolution. The apertures are shown in Fig. C.1.

All flux measurements employed a local background subtraction. The reference annulus extended from $40^{\prime \prime}$ to $60^{\prime \prime}$ outside the original Fellwalker clump footprint. The annulus was thus non-circular and followed the clump shape. All pixels that were closer than $20^{\prime \prime}$ to the footprint of another clump were excluded. We used the median of the remaining annulus pixels as the background estimate and did not explicitly interpolate the background over the aperture.

For comparison and to create a subsample of more compact cores, alternative photometry was carried out using the same reference annuli, but $50 \%$ smaller apertures. These covered the pixels with $\tau(250 \mu \mathrm{m})$ values (estimated using Herschel data, see Sect. 2.2 and Appendix C) above the median value of the original aperture. Apertures smaller than $256 \operatorname{arcsec}^{2}$ (16 pixels with a size $4^{\prime \prime} \times 4^{\prime \prime}$ each) were rejected. In the following, the original

\footnotetext{
6 http://starlink. eao.hawaii.edu/docs/sun255.htx/ sun2 55 se 2 .html
} 
and the high-column-density apertures are called $\tau_{\text {all }}$ and $\tau_{\text {high }}$ apertures, respectively.

The statistical errors of the flux values were estimated based on the standard deviation of the pixel values in the annulus and the sizes of the annulus and the aperture. We took the fact into account that the maps were convolved to a resolution of $40^{\prime \prime}$ and scaled the standard deviation of the pixel values with the square root of the ratio between the area of the measuring aperture (or annulus) and the effective area of the $40^{\prime \prime}$ beam. Because of real surface brightness fluctuations within the reference annuli, the photometric errors tend to be overestimated. To reduce this effect, we removed the signal that is linearly correlated between the bands before estimating the noise. Each band was in turn fitted against a reference, which was the average of all the other bands. The correlated signal predicted by the least-squares fit and the reference data was then subtracted. The emission is not perfectly linearly correlated, mainly because of temperature variations. Therefore, the estimates remain an upper limit of the actual noise. In SED fits, the relative weighting of the bands also took calibration uncertainties into account (4\% and 10\% for SPIRE and SCUBA-2, respectively).

\subsection{SED fits}

We fitted SEDs using three or four bands, combining SCUBA-2 measurements and fluxes extracted from SPIRE maps processed through the SCUBA-2 pipeline. We also fitted the fluxes from the original, unfiltered SPIRE maps without SCUBA-2 data, to see how the SPIRE SEDs are affected by the filtering process itself.

The fitted model of flux density is the modified blackbody (MBB) function

$F(v)=F\left(v_{0}\right) \frac{B_{v}(v, T)}{B_{v}\left(v_{0}, T\right)}\left(\frac{v}{v_{0}}\right)^{\beta}=\kappa(v) B_{v}(v, T) M / d^{2}$,

where $v$ is the frequency, $v_{0}$ the selected reference frequency, $B_{v}$ the Planck law, $\beta$ the opacity spectral index, and $d$ the cloud distance (Hildebrand 1983). The three fitted parameters are the flux density at the reference frequency $F\left(v_{0}\right)$, the dust emission colour temperature $T$, and the spectra index $\beta$. The assumption of a value for the dust opacity per unit mass $\kappa(v)$ enables estimating the clump masses $M$. We assumed a value $\kappa=0.1(v / 1 \mathrm{THz})^{\beta} \mathrm{cm}^{2} \mathrm{~g}^{-1}$ (Beckwith et al. 1990), as was used, for example, in papers of the Galactic Cold Cores project (Juvela et al. 2012; Montillaud et al. 2015) and of the Herschel Gould Belt Surveys (André et al. 2010).

Equation (1) describes the apparent shape of an SED in terms of its colour temperature and apparent spectral index. If the SED is assumed to be connected to real dust properties (such as the intrinsic opacity spectral index) and clump properties (such as the mass), one implicitly assumes that the emission is optically thin and dust is characterised by a single temperature and a single $\beta$ value. The impact of these assumption is discussed further in Sect. 5 .

We used only a subset of all the extracted clumps for the SED analysis (see Sects. 3.1 and 3.2). To avoid clumps near high-noise map boundaries, the centre of the included clumps was required to reside within $5^{\prime}$ of the field centre. We also rejected clumps whose $850 \mu \mathrm{m}$ flux was below $0.1 \mathrm{Jy}$ or where the MBB fit to four bands ( $850 \mu \mathrm{m}$ and the filtered SPIRE data) resulted in poor fits (see below).

\section{Results}

We investigated differences of dust emission properties in dense, potentially prestellar clumps and the surrounding more diffuse clouds. We examined the values of the opacity spectral index $\beta$ of the clumps. We sought evidence of correlated changes between the dust temperature and $\beta$ and a potential wavelength dependence of $\beta$. These could be indications of dust processing during the star formation process or might be directly related to the local physical conditions such as the radiation field and the temperature. We start by examining large-scale dust emission of the selected fields (Sect. 4.1) before concentrating on the compact SCUBA-2 clumps and cores (Sect. 4.2). We compare the fits of different wavelength ranges up to the millimetre regime, examine the effect of the extension to the shorter $160 \mu \mathrm{m}$ wavelength (Sect. 4.3), and check the correlations between young stellar objects and the clump and dust emission properties (Sect. 4.4).

\subsection{Dust spectrum at large scales}

Over the entire fields in which the PGCC clumps reside, we analysed IRAS, Herschel, and Planck observations in the wavelength range $\lambda=100-1380 \mu \mathrm{m}$ (from $3000 \mathrm{GHz}$ to $217 \mathrm{GHz}$ ) to characterise the dust emission at large scales. With IRIS data and the zero-point-corrected Herschel maps, we were able to directly use surface brightness values averaged within a radius of $r=8^{\prime}$ of the centre of the SCUBA-2 fields. Alternatively, we could subtract the background that is estimated as the mean surface brightness in a selected reference area. In that case, we added the uncertainty of the background subtraction. This is described by the covariance matrices that are estimated from the values in the reference region, from the part of the signal that is not linearly correlated between the bands. The procedure to exclude the correlated signal is the same as in Sect. 3.2.

To ensure consistent handling of all bands, the maps were convolved to a common resolution of $6^{\prime}$. We carried out MBB fits using different band combinations to check the consistency of the data and to examine possible wavelength dependencies.

\subsubsection{SEDs of IRAS and Planck data}

Figure 1a shows the colour temperature $T$ and the spectral index $\beta$ calculated from the combination of the $100 \mu \mathrm{m}$ IRAS data and the Planck bands at $857 \mathrm{GHz}, 545 \mathrm{GHz}, 353 \mathrm{GHz}$, or $217 \mathrm{GHz}$ (wavelengths $350 \mu \mathrm{m}, 550 \mu \mathrm{m}, 850 \mu \mathrm{m}$, and $1380 \mu \mathrm{m}$, respectively). There is no subtraction of the local background. The confidence regions plotted in Fig. 1 were calculated with the Markov chain Monte Carlo (MCMC) method, using flat priors with $8 \mathrm{~K}<T<25 \mathrm{~K}$ and $0.5<\beta<3$.

The median values of the $100-850 \mu \mathrm{m}$ fit are $T=15.74 \mathrm{~K}$ and $\beta=1.73$. The addition of longer wavelengths up to $1380 \mu \mathrm{m}$ increases the temperature to $T=15.95 \mathrm{~K}$ and lowers the spectral index to $\beta=1.69$. Because long wavelengths are less sensitive to line-of-sight temperature variations, we might have expected the colour temperature to decrease rather than increase (assuming that the opacity spectral index does not change with wavelength). However, differences are not significant compared to the statistical uncertainties $(\sim 1 \mathrm{~K}$ in temperature and $\sim 0.1$ in the spectral index, see Fig. 1) and might easily be caused by small systematic errors. Figure $1 \mathrm{~b}$ illustrates the effect of $\mathrm{CO}$ corrections on the $100-1380 \mu \mathrm{m}$ fits. The difference in Fig. 1a might be explained if we assume that the $\mathrm{CO}$ corrections are underestimated by some $10 \%$. Although there is no particular reason to suspect that 


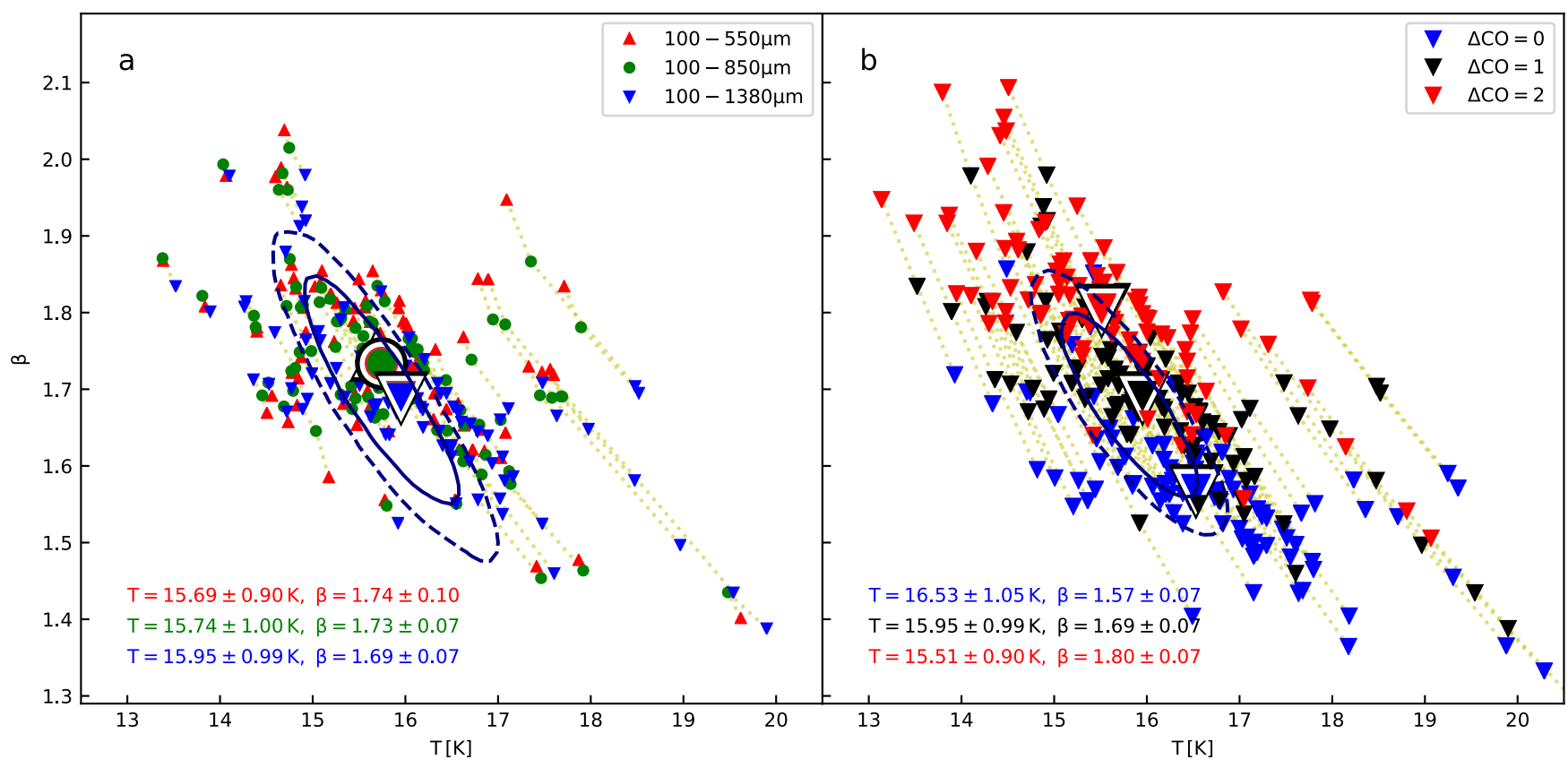

Fig. 1. Results of MBB fits to IRAS and Planck data averaged within $8^{\prime}$ of the centre of the SCUBA-2 fields. Frame a shows the fits completed with $100-550 \mu \mathrm{m}, 100-850 \mu \mathrm{m}$, and 100-1380 $\mu \mathrm{m}$ bands. Each marker corresponds to one field, and the dotted lines connect estimates of the same field. The median values calculated over all the fields are plotted with large symbols. The median values and the $1 \sigma$ dispersion estimated from the interquartile ranges are quoted in the frame. Frame $\mathrm{b}$ shows the fits to the $100-1380 \mu \mathrm{m}$ data without $\mathrm{CO}$ corrections $(\Delta \mathrm{CO}=0)$, with the default $\mathrm{CO}$ correction $(\Delta \mathrm{CO}=1)$, and with twice the default correction $(\Delta \mathrm{CO}=2)$. The median values are indicated by the three large triangles. The contours show the $67 \%$ (solid line) and $95 \%$ (dashed line) confidence regions for a 100-850 $\mu \mathrm{m}$ (frame a) and 100-1380 $\mu \mathrm{m}$ fit with $\Delta \mathrm{CO}=1$ (frame $b$ ) calculated using the median error covariance matrix of the fields.

the correction is underestimated (see below), the evidence for a wavelength dependence of $\beta$ is tentative at most in Fig. 1a.

To further quantify the reliability of the $\mathrm{CO}$ corrections, we compared Planck $\mathrm{CO}$ estimates to ground-based data that provide a partial coverage of about half of the fields. Meng et al. (2013) and Zhang et al. (2016) made CO(1-0) observations at the Purple Mountain Observatory (PMO) radio telescope, fully or partially covering 17 of our SCUBA-2 fields. In Fig. 2b we show the correlation with the Planck $\mathrm{CO}$ estimates after convolving the PMO observations to the same $7^{\prime}$ resolution and scaling with the assumed main beam efficiency of $\eta_{\mathrm{MB}}=0.5$. The correlation is generally good, but the ground-based values are higher on average by $\sim 30 \%$.

The ongoing SAMPLING ${ }^{7}$ (Wang et al. 2018) survey (PI. Ke Wang) at the Arizona Radio Observatory $10 \mathrm{~m}$ SMT telescope is mapping a large number of PGCC targets in the $J=2-1$ transitions of $\mathrm{CO}$ isotopomers and thus provides more direct estimates of the $\mathrm{CO}$ contamination of the $217 \mathrm{GHz}$ band. There are 56 SMT maps near our targets. Because the maps are only $\sim 6^{\prime}$, we used the Planck CO maps at their original resolution of $5.5^{\prime}$ in the comparison. We convolved the SMT data to this resolution and compared values at the centre positions of the SMT maps (Fig. 2a). The SMT values are the sum of the ${ }^{12} \mathrm{CO}(2-1)$ and ${ }^{13} \mathrm{CO}(2-1)$ lines and are plotted against 0.5 times the Planck $\mathrm{CO}(1-0)$ estimates. This corresponds to the assumption of the $(2-1) /(1-0)=0.5$ line ratio that was used in the CO correction of the Planck $217 \mathrm{GHz}$ data. The agreement is very good and shows that the performed CO corrections were at the appropriate level. The convolution of the SMT data was only possible to calculate using the extent of the observed map, which may cause some overestimation of the SMT values (assuming that

\footnotetext{
7 http://sky-sampling.github.io
}

the CO emission decreases with distance from the SCUBA-2 clump). When we assume consistent calibration of the SMT and PMO observations, the comparison of the two frames in Fig. 2 shows that the $\mathrm{CO}$ line ratio $(2-1) /(1-0)$ tends to be below 0.5 . This would agree with the interpretation that most of the objects are very cold and embedded in more extended and CO-bright envelopes. This also suggests low $\mathrm{CO}$ contamination for the SCUBA-2 $850 \mu \mathrm{m}$ data.

\subsubsection{SEDs of Herschel and Planck data}

Because the fields were selected based on the availability of Herschel data, we repeated the analysis using only wavelengths $\lambda \geq 250 \mu \mathrm{m}$. Figure 3 shows the results from the fits to Herschel $(250 \mu \mathrm{m}, 350 \mu \mathrm{m}$, and $500 \mu \mathrm{m})$ and Planck data. The maps were convolved to the resolution of $6^{\prime}$, and the surface brightness was averaged within a radius of $r=8^{\prime}$. We excluded three fields that are only partially covered by Herschel. The relative weighting of the bands follows the uncertainties discussed in Sects. 2.2 and 2.3 , including a $30 \%$ uncertainty for the $\mathrm{CO}$ correction of the two lowest frequency Planck channels.

Results of Fig. 3a were calculated without background subtraction. Because the intensity zero-points of the Herschel maps are based on Planck data, the two data sets are not completely independent. The median values of the SPIRE fits are $T=14.70 \mathrm{~K}$ and $\beta=1.80$. The results are practically identical when the Planck data are included, $T=14.75 \mathrm{~K}$ and $\beta=1.79$. Compared to Fig. 1, the temperatures are lower by $1 \mathrm{~K}$ and the spectral index values are higher by more than 0.05 units. These changes are not larger than the estimated uncertainties given in Figs. 1 and 3.

Figure 3a includes examples of confidence regions. Regardless of the correctness of the magnitude of the error 


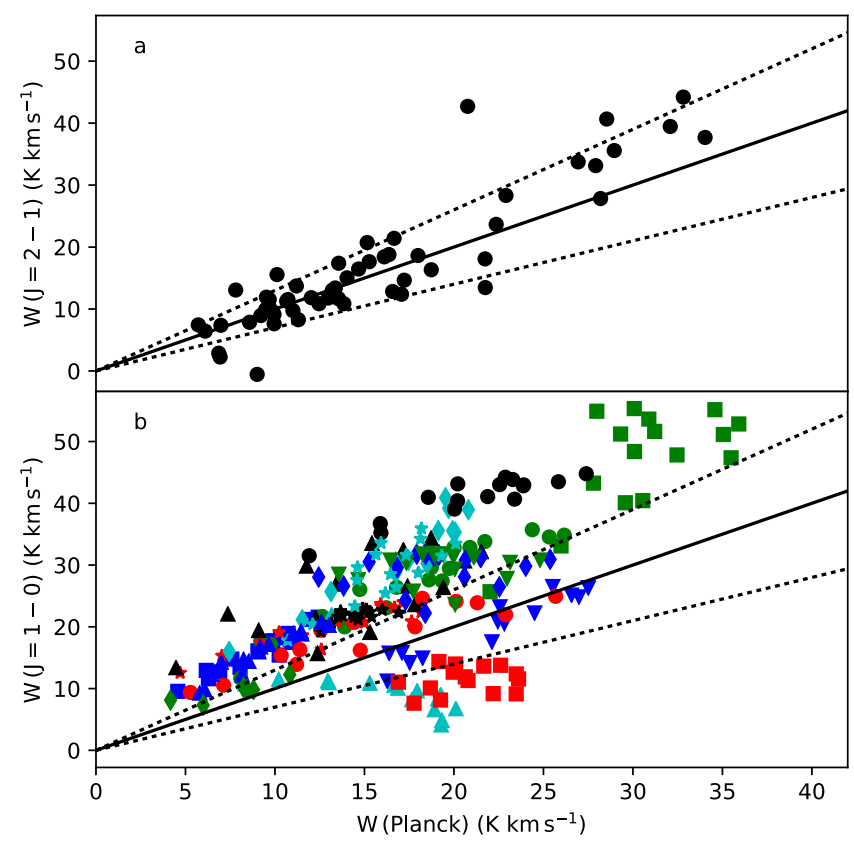

Fig. 2. Comparison of $\mathrm{CO}$ estimates from Planck Type $3 \mathrm{CO}$ maps and ground-based observations. Upper frame compares the sum of ${ }^{12} \mathrm{CO}(2-1)$ and ${ }^{13} \mathrm{CO}(2-1)$ values of 56 SMT maps to Planck $\mathrm{CO}$ estimates for the assumed line ratio of $(2-1) /(1-0)=0.5$. Lower frame shows comparison with $\mathrm{CO}(1-0)$ observations from the PMO telescope, with data at $7^{\prime}$ resolution sampled at $3^{\prime}$ steps. Each colour and symbol combination corresponds to one of 17 fields. Solid lines show the one-to-one relations, and dotted lines correspond to slopes different by $\pm 30 \%$.

estimates, the plot shows the direction along which the $T$ and $\beta$ values are partly degenerate. Figure $3 b$ shows the results when estimates of the local background were subtracted from the flux values. The background region was defined by taking a $24^{\prime} \times 24^{\prime}$ area centred on the SCUBA-2 map and rejecting pixels outside of or closer than $3^{\prime}$ to Herschel map borders. The reference area was defined by the remaining pixels where the $857 \mathrm{GHz}$ surface brightness was below its $10 \%$ percentile. We subtracted the mean surface brightness value of the reference area at each wavelength. The scatter of the $(T, \beta)$ estimates increased, as expected, because of the smaller residual emission above the background. For the median, the $\beta$ value is now lower for the $250-500 \mu \mathrm{m}$ fits and higher for the $250-1380 \mu \mathrm{m}$ fits. This would be contrary to the expectation of $\beta$ decreasing at millimetre wavelengths.

All PGCC clumps with centre coordinates within a radius of $5^{\prime}$ of the SCUBA-2 map centres are marked in the maps of Appendix C. They are also listed in Table H.3, including the PGCC values of the spectral index and the colour temperature (with $\beta=2$ and with free $\beta$ ). The PGCC values result from fits to the cold emission component, after the subtraction of the warm component that is defined by the IRAS $100 \mu \mathrm{m}$ values and the average SED of the region. Thus, the PGCC temperatures are significantly lower than in the analysis above, and the spectral index values are correspondingly higher. For the clumps of Table H.3 for which $T$ and $\beta$ estimates are available, the quartile points $(25 \%, 50 \%$, and $75 \%$ percentiles $)$ are $10.7 \mathrm{~K}, 11.6 \mathrm{~K}$, and $12.3 \mathrm{~K}$ for the colour temperature and $2.01,2.18$, and 2.37 for the spectral index. The $\beta$ values for the PGCC clumps are thus higher than the values of the entire fields (Fig. 1). This is not yet direct evidence of a change in the intrinsic dust properties. By averaging emission over large areas, we also have averaged SEDs with different temperatures, and this can lead to lower apparent $\beta$ values. Therefore, it is necessary to also investigate the spectra at higher spatial resolution.

\subsection{Dust spectrum at small scales}

We investigated the small-scale dust emission using the combination of Herschel 250-500 $\mu \mathrm{m}$ and SCUBA-2 $850 \mu \mathrm{m}$ data and clumps extracted with the Fellwalker method (see Sect. 3.1). We used four clump samples that correspond to (1) $\theta_{\mathrm{F}}=500^{\prime \prime}$ and LM source masks, (2) $\theta_{\mathrm{F}}=200^{\prime \prime}$, SM masks, and $\tau_{\text {high }}$ part of the clumps, (3) $\theta_{\mathrm{F}}=200^{\prime \prime}$ and M850 masks, and (4) data reduction with $\theta_{\mathrm{F}}=200^{\prime \prime}$ and without external source masks. The four alternatives were used to examine the robustness of the results with respect to the details of the data reduction and clump selection.

After the rejection of clumps farther away than $5^{\prime}$ from the field centres or with $S(850 \mu \mathrm{m})$ values below $0.1 \mathrm{Jy}$, there remain about 200 clumps, the number depending on the data version. Many SED fits are not reliable, and we carried out a further selection based on the $\chi^{2}$ values of the fits.

Figure $4 \mathrm{a}-\mathrm{c}$ shows the $\beta$ distributions for the reduction with $\theta_{\mathrm{F}}=500^{\prime \prime}$ and LM masks, using the full clump apertures $\left(\tau_{\mathrm{all}}\right)$. The clumps were divided into subsamples where the $\chi^{2}$ values of the fits were within the best $P\left(\chi^{2}\right)=85 \%, 25 \%, 10 \%$, or 5\%. Thus, $P\left(\chi^{2}\right)=5 \%$ corresponds to the strictest selection criteria (least clumps) with $\chi^{2}$ values below the $5 \%$ percentile point. The first two frames show the results for fits to Herschel $250-500 \mu \mathrm{m}$ bands, before and after filtering by the SCUBA-2 pipeline. The filtering could lead to different results if the filtering procedure introduces additional uncertainty or bias, or if the true SEDs are affected by the removal of large-scale emission.

In the following, we refer mainly to the numbers for the $P\left(\chi^{2}\right)=25 \%$ samples. For the original SPIRE data (the $\theta_{\mathrm{F}}=500^{\prime \prime}$ case), the median value is $\beta=1.90$. Based on the distribution of the $\beta$ values, the error of the mean is smaller than 0.05 . The filtering increases the width of the distributions, partly because of the larger relative uncertainty of the lower flux values and possibly via larger background fluctuations. If the densest parts of the clumps have lower colour temperatures and higher $\beta$ values than their environment, we might expect the filtering to increase in the $\beta$ estimates. Figure 4 shows only weak indications of such a trend.

Figure $4 \mathrm{c}$ shows the results for fits to combined Herschel and SCUBA-2 data, with self-consistent filtering across all bands. The median value has decreased to $\beta=1.63$. The same trend exists for the $P\left(\chi^{2}\right)=5 \%$ sample, although it is not very reliable because of the low number of clumps.

Figure $4 d-f$ shows a second analysis that is a priori very far from the previous case, using a $\theta_{\mathrm{F}}=200^{\prime \prime}$ filter scale, SM masks, and fluxes integrated over the $\tau_{\text {high }}$ apertures. More of the largescale emission is filtered out, and the smaller source mask and clump size further suppress the contribution of extended emission. The number of clumps is lower than in the previous case, and the $\beta$ distributions are also slightly more narrow. Figure 5 shows the SEDs for the clumps in the $P\left(\chi^{2}\right)=5 \%$ sample. In six out of eight cases, the addition of the $850 \mu \mathrm{m}$ has decreased the $\beta$ estimate. Table H.4 lists the fit parameters for the $P\left(\chi^{2}\right)=25 \%$ sample.

Figure $4 \mathrm{~g}-\mathrm{i}$ shows a case where maps were made using M850 source masks and $\theta_{\mathrm{F}}=200^{\prime \prime}$ filtering scale. Compared to the previous cases, the clump selection is thus more directly based on the SCUBA-2 $850 \mu \mathrm{m}$ data. However, the result for the 


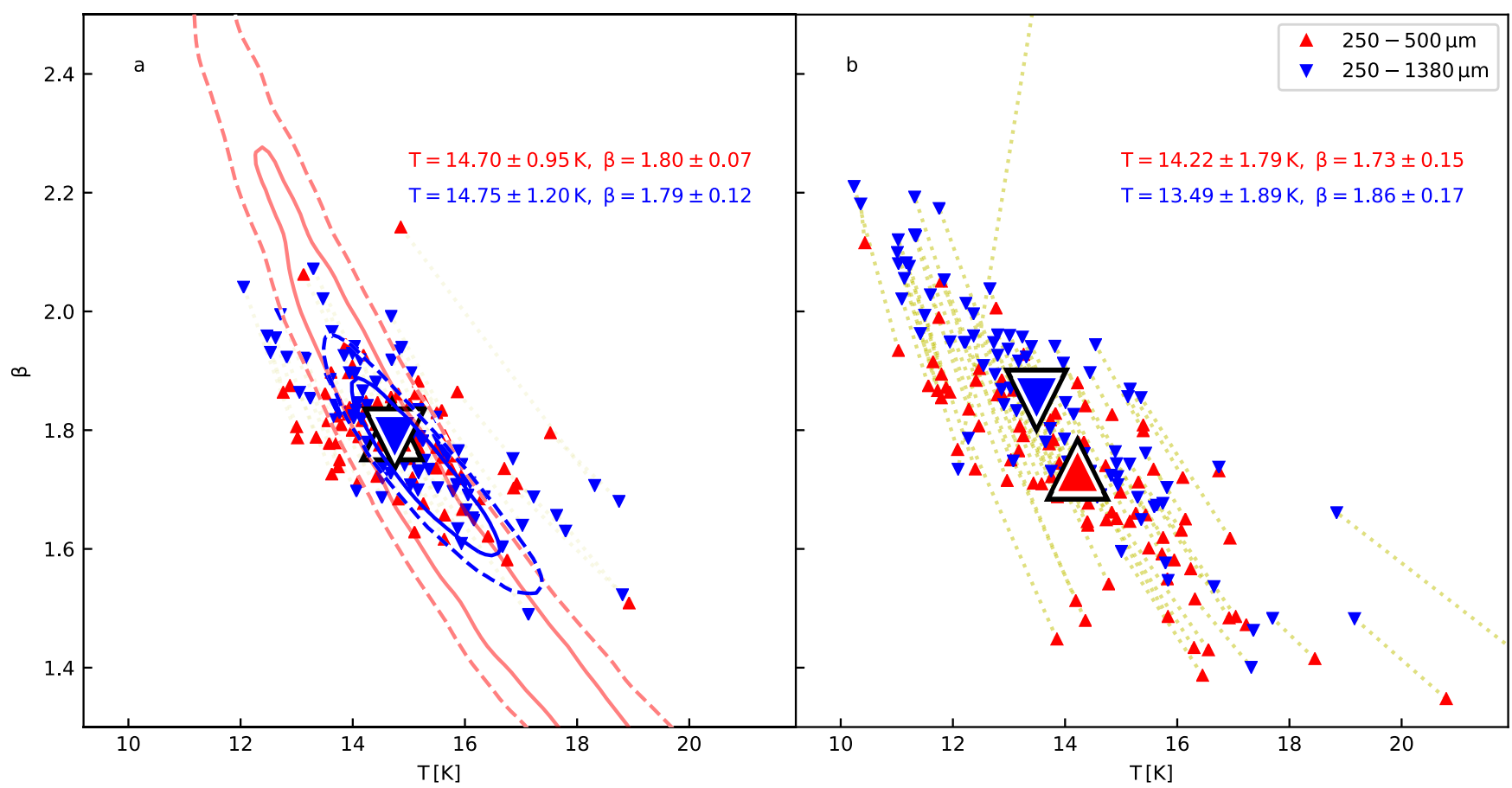

Fig. 3. Results of MBB fits to Herschel and Planck data averaged within $8^{\prime}$ of the centre of the SCUBA-2 fields. The results are shown for absolute surface brightness measurements (frame $a$ ) and after subtraction of the local background emission (frame $b$ ). The symbols correspond to the band combinations listed in the legend in frame $b$. The large symbols denote the median values over the fields. In frame $a$, the contours show the $67 \%$ (solid lines) and 95\% (dashed lines) confidence regions for 250-500 $\mu \mathrm{m}$ (red) and 250-1380 $\mu \mathrm{m}$ (blue) fits, using error covariance matrices that are the median over all fields. For the SPIRE-only fits, the adopted error estimates clearly overestimate the true uncertainty.

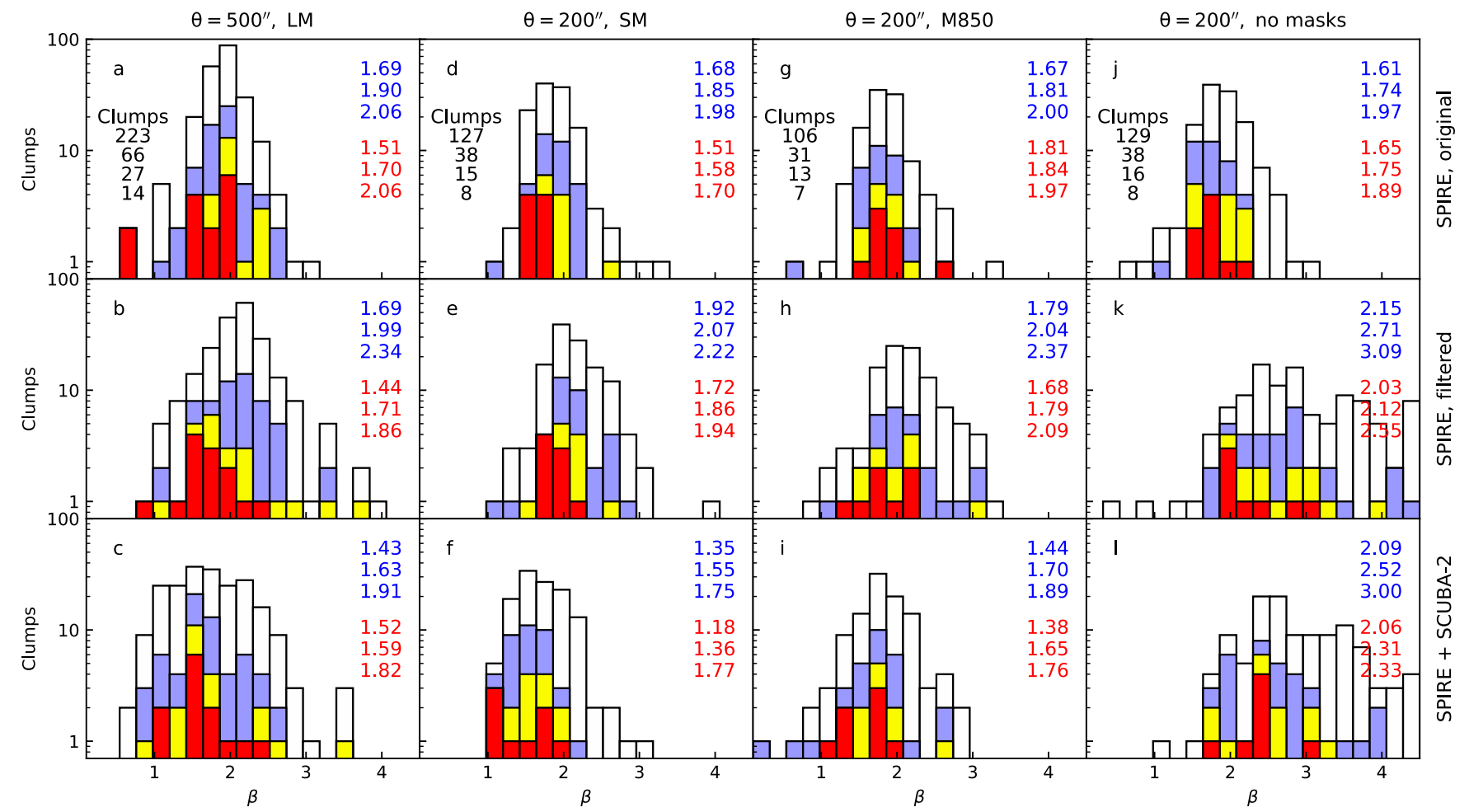

Fig. 4. Distributions of $\beta$ values in fits to Herschel and SCUBA-2 $850 \mu \mathrm{m}$ observations of clumps. Results are shown for original SPIRE data (first row), filtered SPIRE data (second row), and the combination of filtered SPIRE and $850 \mu \mathrm{m}$ data (third row). The columns correspond to different versions of data reduction, as indicated above the frames. The white, blue, yellow, and red histograms correspond to $P\left(\chi^{2}\right)$ values of $85 \%, 25 \%$, $10 \%$, and $5 \%$, respectively (see text). The number of clumps in each sample and the quartile values (25\%, 50\%, and $75 \%$ percentiles) of the blue and red histograms are given in the frames. 


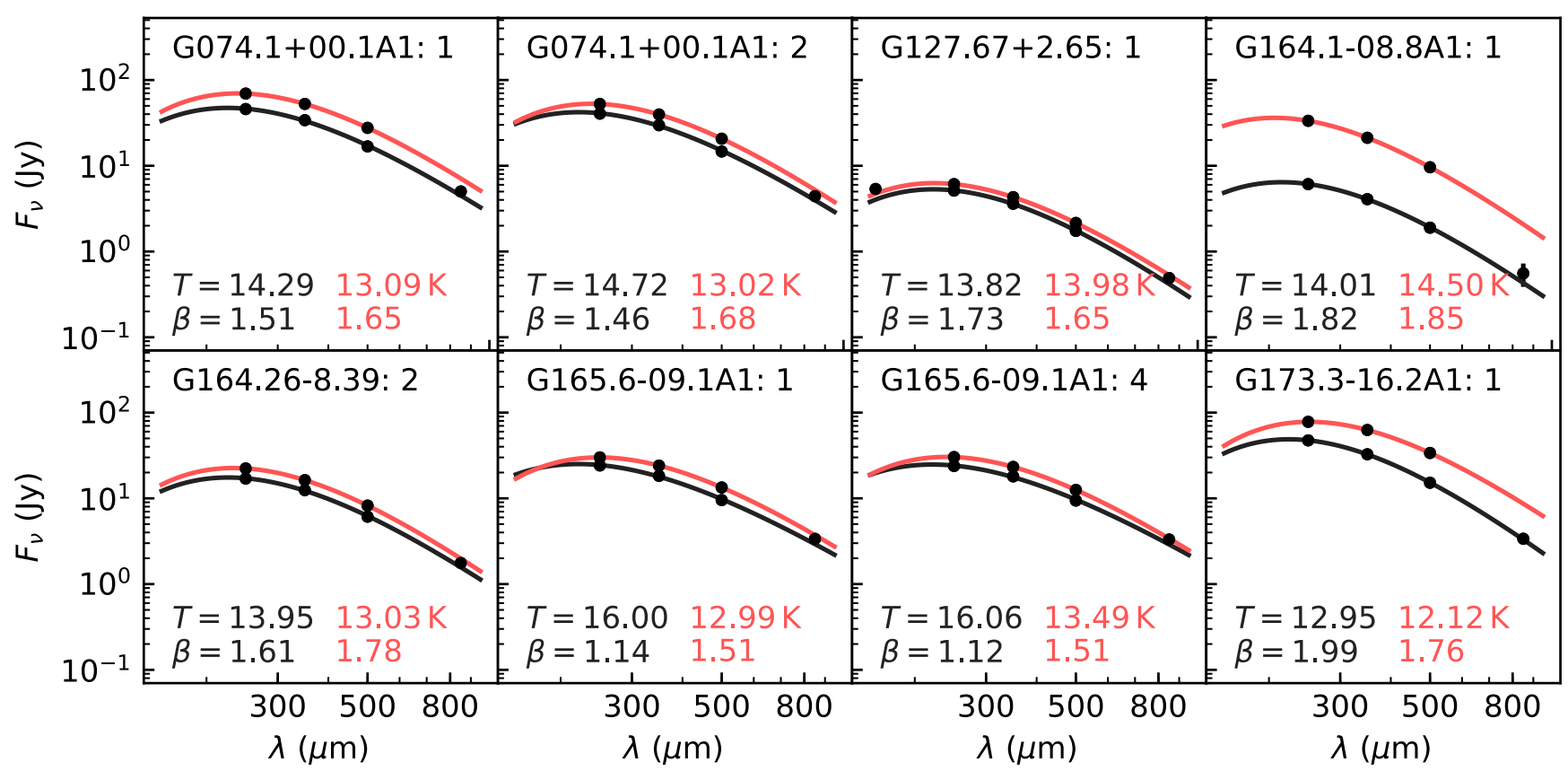

Fig. 5. SEDs of eight sources from the $P\left(\chi^{2}\right)=5 \%$ subsample of SM clumps in Fig. 4f. Plots include fits to the original SPIRE data (upper red curve and red numbers) and to the filtered SPIRE data plus the SCUBA-2 $850 \mu \mathrm{m}$ point. The clump numbers given after the field name are the same as in Fig. C.1. The $160 \mu \mathrm{m}$ data points are available for one of the clumps: it is plotted in the figure, but is not part of the fit.

unfiltered SPIRE data shows that the underlying clump samples are still relatively similar. The inclusion of the $850 \mu \mathrm{m}$ point again decreases the median $\beta$ for both the $P\left(\chi^{2}\right)=5 \%$ and $P\left(\chi^{2}\right)=25 \%$ samples

The final plots in Fig. 4j-1 correspond to maps made with $\theta_{\mathrm{F}}=200^{\prime \prime}$ but without any external masks. This is expected to favour the extraction of the most compact sources, although the total number of sources is not lower than in the previous two cases. When filtering is applied to SPIRE data, the $\beta$ distribution moves towards higher values and becomes wider. We interpret this mostly as a sign of a general increase in uncertainty. In the combined fits of SPIRE and SCUBA-2 data, the spectral index values are also much higher than in the SPIRE-only fits.

A table of the fit parameters is provided in Appendix F, where the uncertainty of the $850 \mu \mathrm{m}$ calibration is also briefly discussed.

Figure 6 uses clumps from Fig. $4 \mathrm{a}-\mathrm{f}$ and compares the fits to the original SPIRE data and to the combination of SCUBA-2 and filtered SPIRE data. Figure 6a shows an example of the confidence regions estimated with the MCMC method. It indicates the direction of the error ellipses, but the size of the confidence region varies from clump to clump. The figure suggests that a large fraction of the anticorrelation between $T$ and $\beta$ can be caused by the observational uncertainties.

The dependence of $\beta$ values on other parameters and directly on error estimates is examined further in Appendices $\mathrm{E}$ and $\mathrm{F}$. The latter section also further quantifies the potential effects of the $850 \mu \mathrm{m}$ calibration uncertainty.

\subsection{SEDs with the $160 \mu \mathrm{m}$ point}

Although 37 fields are partially covered by Herschel PACS observations, the number of clumps with $160 \mu \mathrm{m}$ data is limited. Figure 7 examines the 23 clumps in the $P\left(\chi^{2}\right)=25 \%$ sample of clumps from the maps made with $\theta_{\mathrm{F}}=500^{\prime \prime}$ and LM masks.

The $160 \mu \mathrm{m}$ values are typically above the SPIRE fit (Fig. 7a), which might be expected if each source contains a wide

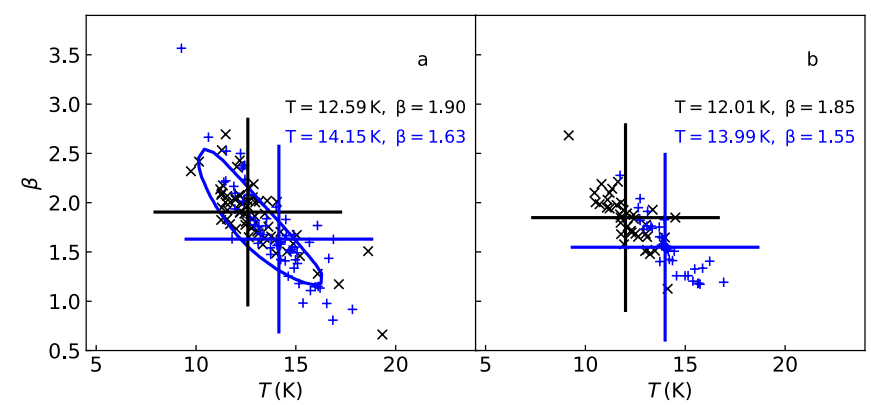

Fig. 6. Spectral index $\beta$ vs. colour temperature $T$ for the clump samples of Fig. $4 \mathrm{a}-\mathrm{c}$ (left frame) and Fig. 4d-f (right frame). The black symbols correspond to fits to the original SPIRE data, and the blue symbols to the combination of SCUBA-2 and filtered SPIRE data. Only clumps from the $P\left(\chi^{2}\right)=25 \%$ samples are included. The median values are indicated with large crosses. Frame a includes a contour for a typical $67 \%$ confidence region in the four-band fits.

range of temperatures. Figure $7 \mathrm{~b}$ compares $(T, \beta)$ values for $\mathrm{Her}$ schel $250-500 \mu \mathrm{m}$ and $160-500 \mu \mathrm{m} \mathrm{MBB}$ fits. Both fits cover a similar region in the $(T, \beta)$ plane, except for a few clumps where the inclusion of the $160 \mu \mathrm{m}$ point leads to much higher temperatures and lower $\beta$ values. The number of these sources is small enough so that the shift in the median parameter values remains small with $\Delta T=+0.4 \mathrm{~K}$ and $\Delta \beta=-0.1$. For simplicity, both fits were carried out without considering error correlations between the different bands. The SPIRE fits are therefore not identical to those in Fig. 4a. The difference in the median value of $\beta$ is 0.08 units.

\subsection{Young stellar objects}

We examined the spatial distribution of young stellar objects (YSOs) using the catalogue of YSO candidates of Marton et al. (2016) that is based on the analysis of 2MASS (Skrutskie et al. 2006) and WISE (Wright et al. 2010) data. 

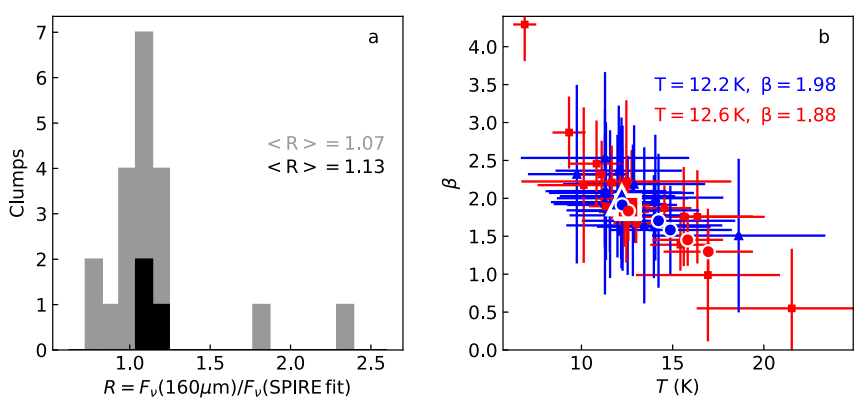

Fig. 7. Comparison of Herschel MBB fits with and without the $160 \mu \mathrm{m}$ data point in the case of the $\mathrm{P}\left(\chi^{2}\right)=25 \%$ clump sample with $\theta_{\mathrm{F}}=500^{\prime \prime}$ and LM masks. The left frame shows the distribution of ratios between the $160 \mu \mathrm{m}$ flux density and the value predicted by the $250-500 \mu \mathrm{m}$ fit. The grey histogram contains all clumps, and the black histogram is based on three clumps with YSO candidates. The second frame shows $(T, \beta)$ values for individual clumps for the $250-500 \mu \mathrm{m}$ (blue triangles) and $160-500 \mu \mathrm{m}$ (red squares) fits. The circles indicate clumps with YSO candidates. The median values are quoted in the frame and are plotted with larger symbols.

Figure 8 shows the surface density as a function of the distance from the centre of the SCUBA-2 fields. The density of Class III candidates is practically constant, about $3-4 \times 10^{-3} \operatorname{arcmin}^{-2}$. The density drops only within the innermost $7^{\prime}$, possibly because of the observational bias resulting from the increasing extinction. Except for this, Class III sources are not correlated with the clumps. The total number of Class I-II sources is higher. Their density also increases significantly towards the centre of the fields, reaching $2.8 \times 10^{-2}$ arcmin $^{-2}$ within the innermost $2^{\prime}$ radius. The highest concentration of Class I-II candidates is found in the field G130.1+11.0A1, where the Herschel coverage is unfortunately incomplete.

We separately calculated the YSO surface densities for all clumps and for the subsample selected for the SED analysis (see Sect. 4.2). For Class I-II candidates, the trend seen in radial profiles continues, and they are found preferentially inside the clumps (see Fig. 8). The surface density is $7.7 \times$ $10^{-2} \operatorname{arcmin}^{-2}$ within the $P\left(\chi^{2}\right)=85 \%$ clump sample. It is also significant that none of the Class III candidates falls inside a clump.

In the LM case, the $P\left(\chi^{2}\right)=25 \%$ sample includes 56 clumps, 5 of which had Class I-II YSO candidates. In SPIRE fits, the YSO clumps have median values of $T=14.5 \pm 0.5 \mathrm{~K}$ and $\beta=1.62 \pm 0.14$, while starless clumps have $T=11.9 \pm 0.9 \mathrm{~K}$ and $\beta=2.1 \pm 0.3$, where the quoted uncertainties are median absolute deviations of the distributions. For the $250-850 \mu \mathrm{m}$ fits, the corresponding values are $T=14.8 \pm 1.0 \mathrm{~K}$ and $\beta=1.58 \pm 0.08$ vs. $T=14.0 \pm 1.2 \mathrm{~K}$ and $\beta=1.59 \pm 0.23$. There is thus a tendency for the proto-stellar clumps to be warmer, while the evidence of lower $\beta$ values is not altogether consistent. The sample of proto-stellar source candidates also comprises only six sources. In Fig. 7 there were only three clumps with YSO candidates that also had PACS observations at $160 \mu \mathrm{m}$. For this small sample, the clump temperatures were also higher than average. The ratio between the $160 \mu \mathrm{m}$ measurement and the fit to the longer wavelength bands was also higher for clumps with YSO candidates. However, the effects of YSO heating appear to be small (Fig. 7a).

The observed differences in the apparent $(T, \beta)$ values do not necessarily mean changes in the intrinsic dust properties. Large temperature variations, such as caused by internal heating, could lower the $\beta$ values estimated from the SEDs.

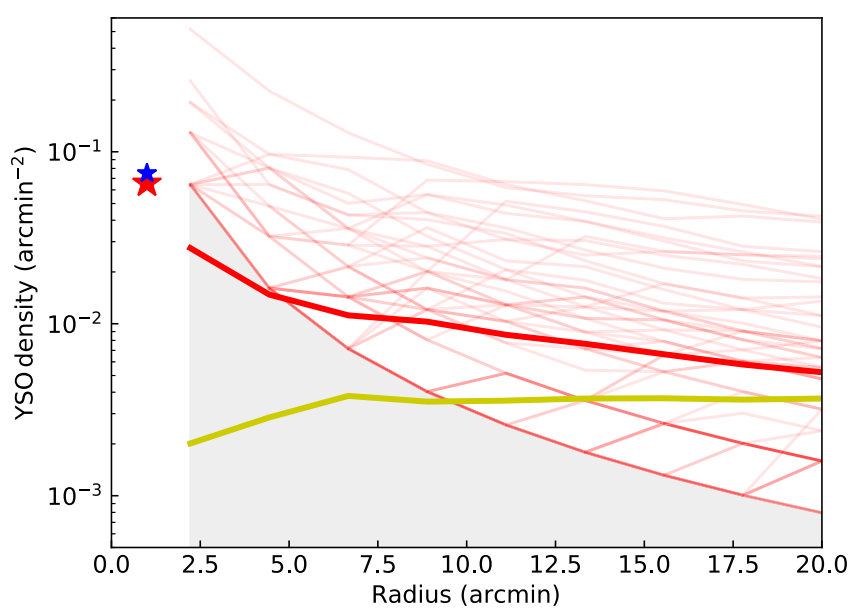

Fig. 8. YSO statistics. The surface density of sources is shown as a function of the distance $\left(>2^{\prime}\right)$ from the field centre. The mean values over all fields are shown for Class I-II (thick red line) and Class III (thick yellow line) candidates. The thin red lines correspond to Class I-II sources in individual fields. The shading corresponds to a region with less than one source for a given distance. The two overlapping stars indicate the density of Class I-II candidates in all clumps (all fields) selected by the Fellwalker algorithm (red star) and in clumps belonging to the $P\left(\chi^{2}\right)=85 \%$ sample (blue star).

\subsection{Clump masses}

Most fields have distance estimates in Table H.2, which allows calculating the physical size, mass, and volume density of the clumps. Figure 9 shows results for the LM data, the $P\left(\chi^{2}\right)=85 \%$ clump sample. The masses correspond to fluxes inside measurement apertures of area $A$, and the volume densities are calculated for a spherical volume with an effective radius $r$ for which $A=\pi r^{2}$.

The mass and distance are correlated because the clump detections are affected by our sensitivity to low flux densities. In Fig. 9a the dashed line corresponds to a mass threshold that assumes a $3.4 \mathrm{mJy}$ beam $^{-1}$ noise level and a $5 \sigma$ detection over a single $850 \mu \mathrm{m}$ beam. The dotted line corresponds to a $850 \mu \mathrm{m}$ flux density of $0.1 \mathrm{Jy}$ for both the $T=13 \mathrm{~K}$ and $\beta=1.8$ spectrum. The correlation in Fig. 9a thus mainly reflects the flux density threshold combined with the geometrical effect of the distance. All sources are relatively close to the threshold, and we do not have nearby sources that would also be very massive. This is natural because low-mass objects are generally much more numerous, and on the other hand, the spatial filtering of the SCUBA-2 data and the clump detection algorithm both limit the possibility of detecting very extended sources. The massdistance relation is also affected by the large uncertainty of the distances and the functional dependence between the distance and the mass estimates.

The temperature is not dependent on the field distance, but there is some negative correlation between the volume density and the clump temperature. However, negative errors in temperature will naturally lead to positive errors in column density, mass, and volume density. Higher temperature also directly enables the detection of lower density objects. Furthermore, the fluxes were measured from apertures that were extended $20^{\prime \prime}$ beyond the original SCUBA-2 clump detections in order to accommodate the lower resolution of the SPIRE data. The modified clump size can introduce some bias that is also dependent on the temperature. For the same sample of clumps, the correlation between the temperature and the column density remains very weak. 

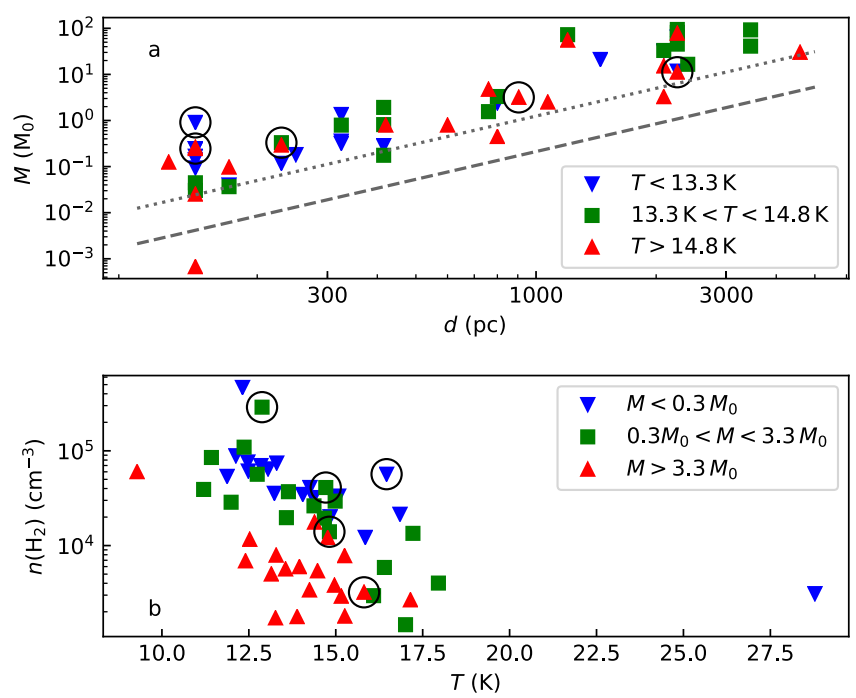

Fig. 9. Clump mass vs. field distance (frame a) and average volume density vs. temperature (frame $b$ ). The plots correspond to the LM data version and the $P\left(\chi^{2}\right)=25 \%$ clump sample. The fluxes and temperatures are from the $250-850 \mu \mathrm{m}$ fits. The data are further divided into three samples based on temperature (frame $a$ ) or mass (frame $b$ ). The clumps with YSO Class I or II candidates are marked with circles. Frame $a$ shows the thresholds corresponding to a $17 \mathrm{mJy}$ per $850 \mu \mathrm{m}$ beam (dashed line) and to a $850 \mu \mathrm{m}$ flux density of $0.1 \mathrm{Jy}$ (dotted line; see text).

We have marked the clumps with Class I or II YSO candidates in the plot. The median distance of YSO sources is smaller than that of the full clump sample, but this is not significant considering the small number of YSO candidates. As noted in Sect. 4.4, there is some tendency for proto-stellar clumps to be warmer than clumps on average. In Fig. 9, the proto-stellar clumps do not stand out based on either their mass or density.

We note that while clumps with embedded proto-stars may be typically among the warmer cores in the sample, they obey a similar temperature/density relation as the starless clumps. This is in contrast to the behaviour seen on the scale of individual star-forming cores (with sizes $\sim 10^{-2} \mathrm{pc}$ ): combined SCUBA-2 and Herschel observations of starless cores in the Taurus molecular cloud (Ward-Thompson et al. 2016) and in the Cepheus Flare (Pattle et al. 2017) have shown that cores heated by nearby or embedded protostars are significantly warmer than cores of the same density that are not affected by proto-stars. The lack of a systematic elevation in temperature in Planck clumps with embedded proto-stars relative to starless Planck clumps of similar densities suggests that the heating effect of a protostar on its environment is limited to a relatively small volume surrounding the proto-star itself, and so that embedded proto-stars do not significantly raise the average dust temperature of their parent clumps.

\section{Discussion}

We have investigated submillimetre dust emission in 96 fields observed with the SCUBA-2 instrument at $850 \mu \mathrm{m}$ and with at least partial Herschel coverage. In the following, we discuss the results obtained at large scales, based on IRAS, Planck, and Herschel data, and at small scales, based on the combination of Herschel and SCUBA-2 observations.

\subsection{Dust SEDs of PGCC host clouds}

PGCC sources represent the coldest part of the interstellar medium (ISM), and the environments examined in Sect. 4.1.2 should therefore consist mostly of parts of molecular clouds. This is confirmed by the column densities. Based on IRAS and Planck data at $100-550 \mu \mathrm{m}$ and the dust opacity adopted in Sect. 3.3, the average values are $\mathrm{N}\left(\mathrm{H}_{2}\right)=3.5 \times 10^{21} \mathrm{~cm}^{-2}$ within the $r=8^{\prime}$ annuli, but only $1.1 \times 10^{21} \mathrm{~cm}^{-2}$ immediately outside this region. Typical SEDs measured with IRAS and Planck data correspond to a colour temperature of $T=15.7 \mathrm{~K}$ and a dust opacity spectral index of $\beta=1.7$, with some variations depending on the actual bands used (see Fig. 1). Most colour temperature estimates are within $1 \mathrm{~K}$ of the quoted median values.

When the IRAS $100 \mu \mathrm{m}$ point is replaced with $250-550 \mu \mathrm{m}$ Herschel measurements, the median colour temperature is about $14.7 \mathrm{~K}$ and the spectral index is close to $\beta=1.8$ (see Fig. 3a). Bootstrapping gives purely statistical uncertainties of $0.13 \mathrm{~K}$ in temperature and 0.015 in $\beta$ (sample median values). The difference between the fits could be affected by very small grain emission in the $100 \mu \mathrm{m}$ band or by the fact that wavelengths below the emission peak are more sensitive to warm dust and lead to lower $\beta$ estimates when the temperature varies. When the analysis was repeated with background-subtracted flux measurements (Fig. 3b), the spectral index estimate remained similar, which could support the latter explanation. However, we also repeated the fits using only Planck data. In spite of the absence of short wavelengths $\lambda<350 \mu \mathrm{m}$, these resulted in lower spectral index values $\beta \sim 1.6$ (see Appendix D). This result is, of course, affected by larger uncertainties. We note that in Malinen et al. (2014), the use of different wavelength ranges (from 100-500 $\mu \mathrm{m}$ to $160-1380 \mu \mathrm{m})$ did not result in significant changes in the $(T$, $\beta$ ) estimates of the proto-stellar and starless cores of the cloud LDN 1642 either.

The colour temperatures of the selected fields are lower and the spectral indices higher than in the general ISM. For example, Planck Collaboration Int. XIV (2014) reported in the $100-850 \mu \mathrm{m}$ range values $\beta=1.54$ for regions dominated by atomic gas and $\beta=1.66$ for mostly molecular clouds (see also Planck Collaboration X 2016). That our SEDs are somewhat steeper (with higher $\beta$ ) reflects the fact that the selected fields represent the densest parts of molecular clouds. Juvela et al. (2015a) found $T=16.1 \mathrm{~K}$ and $\beta=1.84$ for a sample of PGCC field, also using the combination of IRAS and Planck bands at $100-850 \mu \mathrm{m}$. The values are comparable to those derived for the present sample, although the median spectral index in Fig. 1 is slightly lower, $\beta=1.73$. The values also depend on the relative weights given for the fitted bands, which are not identical between these two studies. In the present study, the weight of the $857 \mathrm{GHz}$ and $545 \mathrm{GHz}$ relative to both IRAS and lower frequency Planck bands was higher than in Juvela et al. (2015a).

The results of Fig. $3 a$ and $b$ could be affected by temperature variations within the apertures. By removing the warmer diffuse component, background subtraction should make the remaining emission more isothermal, which could in turn increase the observed $\beta$ values. However, no such effect was observed.

\subsection{SEDs of SCUBA-2 clumps}

Dust emission of dense clumps and cores was examined with Herschel and SCUBA-2 data. Depending on the $P\left(\chi^{2}\right)$ value, the average clump area of the samples is 2.1-2.5 $\operatorname{arcmin}^{2}$ or $0.19-0.3 \mathrm{pc}^{2}$. The spectral index distributions were examined in Fig. 4 for alternative reductions, clump selections, and wavelength ranges. These comparisons help to establish the full uncertainty of the results that may not be captured by the a formal photometric and calibration errors alone. In spite of possible 
differences in the reliability (partially reflected in the width of the distributions), the main results are relatively robust. Most estimates of the submillimetre spectral index are in the range $\beta=1.6-1.9$. The $850 \mu \mathrm{m}$ data point has a clear effect on the spectral index estimates. While the fits to the SPIRE data gave median $\beta$ values slightly above 1.8 , the joint fits of SPIRE and SCUBA- $2850 \mu \mathrm{m}$ decreased these to $\beta \sim 1$.6. However, as demonstrated by Fig. 41, there can be error sources that are not yet all fully understood.

The SPIRE fits can be compared with the results of Juvela et al. (2015a). The spectral index values there were higher at a scale of $10^{\prime}$. The median estimates were $T=14.9 \mathrm{~K}$ and $\beta=2.04$ compared to the values $T=14.7 \mathrm{~K}$ and $\beta=1.8$ in Fig. 3a. Juvela et al. (2015a) did not report statistics on dense clumps, but presented $T$ and $\beta$ maps, which sometimes showed clear spatial $\beta$ variations with maximum values rising above $\beta=2.0$. This was true mostly for well-resolved nearby clumps. The median distance of the fields in Juvela et al. (2015a) was 620 pc. In this paper the median distance of the fields is only $230 \mathrm{pc}$, but, as shown by Fig. 9, most of the detected clumps are at larger distances. The nearby fields tend to be at higher Galactic latitudes, have lower column densities, and therefore have fewer SCUBA-2 detections. Based on Juvela et al. (2015a), we can expect significant dispersion in the $\beta$ histograms. The $\beta$ estimates are in the range $1.0<\beta<2.5$, and we found no real evidence for extreme values of either $\beta \leq 1$ or $\beta \geq 3$.

Data at wavelengths below $250 \mu \mathrm{m}$ were mostly excluded from the analysis because of the incomplete coverage of the SCUBA-2 fields and because of the potential effects of temperature mixing. In Sect. 4.3 the addition of the $160 \mu \mathrm{m}$ point changed the median values by $\Delta T=+0.4 \mathrm{~K}$ and $\Delta \beta=-0.1$. The exact values again depend significantly on the relative weighting of the different bands (see e.g. Shetty et al. 2009b).

The $\beta$ values derived in this work can be compared with recent results on starless and proto-stellar clumps. Sadavoy et al. (2013) analysed Herschel and SCUBA-2 observations of Perseus (clump B1) and found typical spectral index values of $\beta \approx 2$. Towards proto-stellar sources, the values were lower, but still mostly above $\beta=1.6$. The values are thus similar to this study, although we did not observe a significant difference between clumps with and without YSO candidates that could be interpreted as signs of systematic dust evolution (Fig. 7). Chen et al. (2016) investigated the Perseus cloud further and found notable differences between the various cloud regions. The pixel-bypixel histograms covered the range $\beta \sim 1.0-2.7$. The lower $\beta$ values were found to be correlated with local peaks of colour temperature, but also with $\mathrm{CO}$ outflows and thus directly with YSOs. This still raises the question to what extent the changes in the observed $\beta$ values are the result of dust evolution or of the more complex temperature structure of the sources. Bracco et al. (2017) mapped spectral indices of the Taurus B213 filament, including both pre-stellar and proto-stellar clumps. The observations consisted of $1.15 \mathrm{~mm}$ and $2.0 \mathrm{~mm}$ ground-based observations that were analysed together with temperature constraints obtained from Herschel. A pre-stellar core was found to have a high spectral index of $\beta \sim 2.4$ that also remained radially constant. Because the values resulted from a 3D inversion, they are already corrected for the line-of-sight temperature mixing and should thus be somewhat higher than values dirctly derived from the SEDs. In the case of proto-stellar sources, the inversion of the radial profiles suggested a decrease from $\beta \sim 1.5-2.0$ in the outer parts to values 0.5 lower in the warmer centre. The lowest values at a core centre were $\beta \sim 1.0$, but the values derived from the basic SED analysis would be expected to remain significantly higher. Li et al. (2017) specifically examined a sample of Class 0 YSOs with interferometric observations from submillimetre to centimetre wavelengths. Most sources exhibited values $\beta<1.7$ (down to $\beta \sim 0.6$ ). The authors noted that in addition to grain growth, the result may be affected by a strong temperature mixing when the high-resolution observations probe the emission from the circumstellar disk. The comparison with the Herschel and SCUBA-2 results is also affected by the difference of the wavelength ranges (if submillimetre SEDs are generally steeper) and the interferometric observations filtering out much of the extended emission.

In summary, our results of $T \sim 14 \mathrm{~K}$ and $\beta \sim 1.6$ for the joint SPIRE and SCUBA-2 fits are very similar to the previous studies. However, in our sample, the difference between starless sources and proto-stellar sources (clumps with YSO candidates) is small. Our YSO statistics may also be incomplete and affected by the sensitivity and resolution of the WISE survey relative to the data used in the other studies.

In our study, data reductions with different Herschel-based source masks and different filtering scales in Fig. 4a-i gave relatively consistent results. Larger filter scales and larger source masks resulted in the recovery of more $850 \mu \mathrm{m}$ extended emission. The maps generally agreed well with the SPIRE observations, which are sensitive to much lower column densities. The use of $\theta_{\mathrm{F}}=500^{\prime \prime}$ and large LM masks leads to more than twice as many clump detections as the combination of $\theta_{\mathrm{F}}=200^{\prime \prime}$ and $\mathrm{SM}$. On the other hand, large masks can sometimes increase random fluctuations in the maps. For example, in Fig. C.1, the main clump of G167.215.3A1 is associated with a negative feature that is located inside the LM mask. The feature disappears when a smaller filter scale (200" instead of $\left.500^{\prime \prime}\right)$ or a tighter source mask (SM instead of LM) is used. While a large filter scale and liberal source masks work well in statistical studies, one may wish to be more conservative when studying individual objects.

\subsection{Wavelength dependence of the spectral index}

One of the main goals of this paper was to investigate the potential flattening of dust SEDs towards millimetre wavelengths. In this respect, the most interesting comparisons are the fits with and without the Planck $217 \mathrm{GHz}$ band $(1380 \mu \mathrm{m})$, and at smaller scales, the fits with and without the SCUBA-2 $850 \mu \mathrm{m}$ band.

According to the IRAS and Planck analysis in Fig. 1, the $100-1380 \mu \mathrm{m}$ fit leads to a marginally lower value of the spectral index $(\beta=1.69)$ than the fits at $\lambda \leq 550 \mu \mathrm{m}(\beta=1.74)$ or $\lambda \leq 850 \mu \mathrm{m}(\beta=1.73)$. The corrections for line emission in the $217 \mathrm{GHz}$ and $353 \mathrm{GHz}$ bands was identified as the main source of uncertainty. Based on Fig. 1, such small differences in $\beta$ could be explained by a systematic $10 \%$ error in the CO corrections. The comparison with the sum of the ground-based ${ }^{12} \mathrm{CO}(2-1)$ and ${ }^{13} \mathrm{CO}(2-1)$ measurements showed that the Planck $\mathrm{CO}$ estimates, which were used to correct the continuum data, are correct to within $\sim 30 \%$ and do not show any significant bias (Fig. 2a). However, the possibility of systematic errors at $10 \%$ level cannot be excluded. The calibration accuracy of ground-based observations is also not much better than this. Considering the uncertainty of the line contamination, which may include contributions of other lines as well (Planck Collaboration X 2016), and because of other potential systematic errors, the change of $\beta$ as a function of wavelength was not significant.

When the same analysis was based on the combination of Herschel and Planck data, the $217 \mathrm{GHz}$ band caused no significant change in $\beta$ (Fig. 3a). Furthermore, in the backgroundsubtracted measurements, the $\beta$ value was higher when the 
$217 \mathrm{GHz}$ band was included. This shows that if $\beta$ does change with wavelength, the effect is at most at the level of the observational uncertainties.

The analysis of Herschel and SCUBA-2 data gave clear indications that the $850 \mu \mathrm{m}$ point would favour lower $\beta$ values (Figs. 4 and 5). The difference with and without the $850 \mu \mathrm{m}$ point resulted in a change of about $\Delta \beta \sim 0.2$, but the result may still depend on the details of the data reduction, as indicated by Fig. $4 j-1$. On the other hand, a relative calibration error of $10 \%$ between SPIRE and SCUBA-2 (or similar CO correction of the $850 \mu \mathrm{m}$ band) would correspond to a change of only $\Delta \beta= \pm 0.03$ in our fits.

\subsection{Relation between $T$ and $\beta$}

The dependence between dust temperature and spectral index is important because it is related to dust evolution during the star formation process and is a potential tracer of the past history and current physical state of clumps. The simultaneous determination of $T$ and $\beta$ is difficult because observational noise produces anticorrelation between the parameters. Moreover, temperature variations within the beam make the observed colour temperature a biased estimate of the physical grain temperature, and spectral index estimates will be similarly biased.

It is therefore not surprising that the observed relations in Figs. 1, 3, and 6 all exhibit some negative correlation between $T$ and $\beta$. In the following, we examine whether these can be caused by noise alone. It is more difficult to make the final connection between the apparent and the real dust properties. This would require detailed modelling and is beyond the scope of this paper.

We first investigate the SEDs of IRAS and Planck data shown in Fig. 1a. Fits of a function $\beta(T)=A \times T^{B}$ (see Désert et al. 2008; Juvela et al. 2015a) give for $B$ values of -0.53 and -0.69 for the $100-550 \mu \mathrm{m}$, and $100-1380 \mu \mathrm{m}$ data, respectively. We examined whether the data are consistent with a constant $\beta$ value, the negative correlation being caused by noise alone. We fitted the data of each field with an MBB function $B_{\nu}(T) \times v^{1.7}$ and rescaled the flux values onto this SED curve. In other words, at this point, the simulated photometry exactly matches the assumed $\beta(T)$ law. We then added photometric noise to the flux density values (see below) and determined the apparent $T$ and $\beta$ values with new fits of the simulated SEDs. In the above we assumed $B_{v}(T) \times v^{1.7}$ spectra, but because the goal is to simulate the relative difference between the true and the observed SEDs, the procedure is not sensitive to the selected value of $\beta$. The results remain essentially identical when a value of $\beta=1.4$ or $\beta=2.0$ is used instead of $\beta=1.7$

Because a direct comparison between observations and simulations would require precise knowledge of the uncertainties, we included the noise level as an additional parameter $k$ (noise). Thus we used the same error covariance matrices as in Sect. 4.1.1, but scaled all the matrix elements with the square of $k$ (noise). We examined the effect of $k$ (noise) on the $\beta(T)$ relation, and on the other hand, the scatter around the fitted $\beta=A \times T^{B}$ curve. Comparison with the observed scatter should provide the most direct empirical upper limit on the noise effects. It is an upper limit because the real emission cannot be expected to follow a single $\beta(T)$ relation, and thus only part of the observed scatter is caused by noise.

For the $100-550 \mu \mathrm{m}$ fits (Fig. 10a, c), the simulations match the observed scatter over a range of $k$ (noise) values, where the value of the $B$ parameter of the $\beta(T)$ relation is also similar to the observed value. The data are thus compatible with a constant spectral index. The situation is different for the fits to
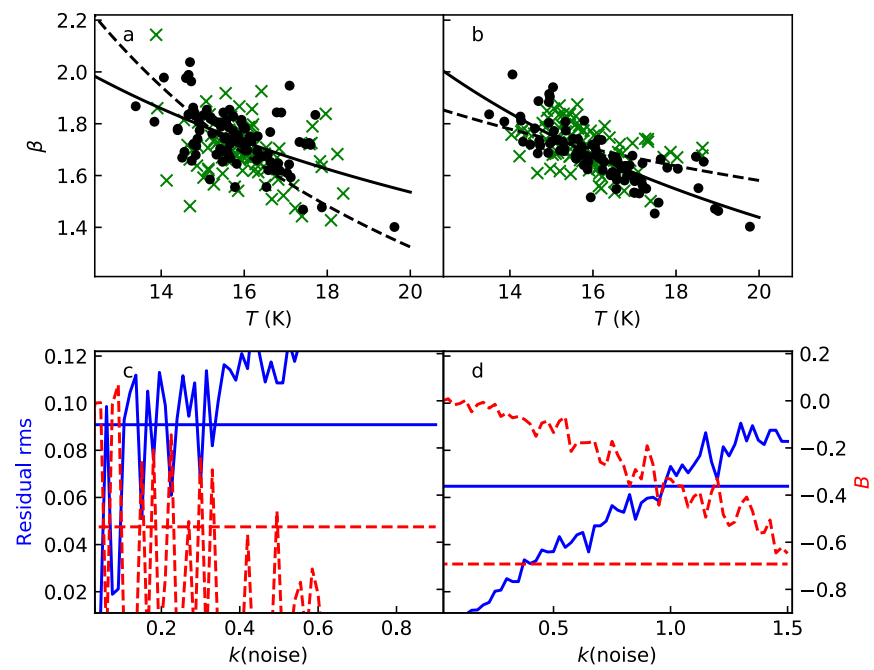

Fig. 10. Comparison of $(T, \beta)$ relations in observations and in simulations with a constant value of $\beta=1.7$. The observations consist of the same IRAS and Planck data as in Fig. 1 and represent the average emission of the fields. Left and right frames show results for $100-550 \mu \mathrm{m}$ and $100-1380 \mu \mathrm{m}$ fits, respectively. In the upper frames, black symbols show the real observations, and green crosses show one realisation of simulations with $k$ (noise) equal to 0.4 (left frame) and 1.0 (right frame). The black lines are fits $\beta=A \times T^{B}$ to the observations (solid line) and to the green crosses (dashed line). The lower frames show the change in the rms value of the residuals of the $\beta(T)$ fit (solid blue lines, left axis) and the value of $B$ (dashed red lines and right axis) as a function of $k$ (noise). The horizontal lines correspond to the values in the actual observations.

$100-1380 \mu \mathrm{m}$ data, which have a smaller uncertainty on the $\beta$ parameter (Fig. 10b, d). The observed scatter is matched near $k$ (noise) $=1.0$, at which point $B$ is -0.35 instead of the value $B=-0.69$. The result would thus suggest that the intrinsic $\beta(T)$ relation is indeed a decreasing function of temperature. Half of the apparent decrease would be caused by noise, but only assuming that the intrinsic $\beta(T)$ relation itself has no scatter. The result remains qualitatively the same, although with different $k$ (noise) values, if the relative weighting of the bands is modified (such as doubling the error estimates of the IRAS band or of the two longest wavelength Planck bands). Figure 10 contains some inconsistency because the $100-550 \mu \mathrm{m}$ fits suggest a lower value of $k$ (noise). However, if the relative error estimates of the $353 \mathrm{GHz}$ and $217 \mathrm{GHz}$ bands are increased, it is possible to fit both $100-550 \mu \mathrm{m}$ and $100-1380 \mu \mathrm{m}$ ranges even with the same value of $k$ (noise).

In Fig. 11 we show the same test applied to the combination of Herschel and Planck data (cf. Fig. 1a). In the simulations, the noise was generated using the same error covariance matrices as in Sec. 4.1.2, but scaling the elements with $k$ (noise) squared. For this shorter wavelength baseline of $250-850 \mu \mathrm{m}$, the observed scatter is reproduced around $k$ (noise) $\approx 0.45$, at which point the predicted $B$ is only slightly above its observed value. For the longer wavelength baseline $250-1380 \mu \mathrm{m}$, the scatter and observed $B$ can both be reproduced with $k$ (noise) $=0.8$, and the data are consistent with a flat intrinsic $\beta(T)$ relation. This does not completely exclude a decreasing $\beta(T)$ function if the clumps do have some real scatter relative to the average $\beta(T)$ relation.

For the clumps, we can only use the shorter wavelength baseline provided by Herschel and SCUBA-2 data. The error estimates that were used to derive the confidence regions in Fig. 6 may have been too large because the $67 \%$ contour already covers almost all of the observations. Nevertheless, the figure 

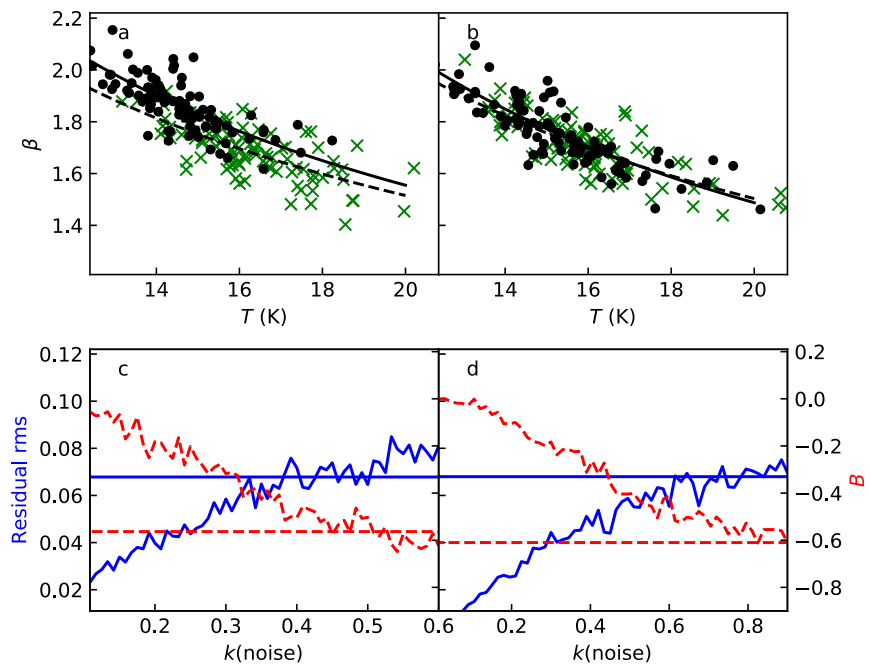

Fig. 11. Same as Fig. 10, but for the combined Herschel and Planck data from Fig. 3. The fitted data are $250-850 \mu \mathrm{m}$ (frames $a$ and $c$ ) and $250-1380 \mu \mathrm{m}$ (frames $b$ and $d$ ). The green crosses correspond to one realisation with $k($ noise $)=0.4$ (frame $a)$ and $k($ noise $)=0.7$ (frame $b)$.

shows that the observed distribution of points is consistent with the orientation of the error ellipse, and the observed distribution of $(T, \beta)$ points might in principle be caused by noise.

Figures 10 and 11 only weakly support the $T-\beta$ anticorrelation, although by attributing all the observed $(T, \beta)$ scatter to observational errors, we underestimate the probability of a decreasing $\beta(T)$ relation. The results at least exclude the possibility of an increasing (apparent) $\beta(T)$ relation.

If the intrinsic $\beta$ of dust grains is constant, the apparent values (derived from the SEDs) could still appear to be positively correlated. This is the case for samples of externally heated clumps of different masses (see e.g. Juvela \& Ysard 2012b, Fig. 6). If the clumps have temperature variations caused by proto-stellar sources, they tend to cause a negative correlation between the observed (apparent) $T$ and $\beta$ values (Shetty et al. 2009b; Malinen et al. 2011; Juvela \& Ysard 2012b). When the noise effects are excluded, this would still not constitute proof of similar behaviour for the actual dust opacity spectral index of the dust grains if we were to conclude that the observation exhibits a negative correlation between the apparent $T$ and $\beta$ values. Figure $10 \mathrm{~b}$ gave the strongest indications of an apparent $T-\beta$ anticorrelation, but because the $100 \mu \mathrm{m}$ data were included, the result may be affected by temperature variations within the aperture or more directly by the emission of very small grains.

The effect of noise on the $\beta(T)$ relation and the joint estimation of the noise level and the $\beta(T)$ relation are examined further in Appendix G.

\section{Conclusions}

We have used IRAS, Planck, Herschel, and SCUBA-2 observations to examine the dust emission spectra in 96 fields that were originally selected based on the presence of prominent PGCC cold clumps. SCUBA-2 data, in conjunction with Herschel SPIRE observations, were used to examine the submillimetre spectra of the densest clumps and cores. The study led to the following conclusions:

- The fields have typical colour temperatures of $14-18 \mathrm{~K}$ and spectral index values of $\beta=1.5-1.9$.
- The clumps extracted from SCUBA-2 maps are characterised by median values of $T \sim 13 \mathrm{~K}$ and $\beta \sim 1.7$. Different options in the data reduction lead to uncertainty at a level of $\delta \beta \sim \pm 0.2$.

- The use of large source masks and large filter scales was successful in recovering more extended emission in the $850 \mu \mathrm{m}$ SCUBA-2 maps. This also resulted in a larger number of clump detections. Because very extended masks can lead to undesirable map fluctuations in individual maps, they may be most useful in statistical studies.

- At large scales, there is no consistent proof for the dust spectrum becoming flatter at millimetre wavelengths. We are limited by the uncertainty of the corrections for $\mathrm{CO}$ line emission in the $353 \mathrm{GHz}$ and $217 \mathrm{GHz}$ Planck bands.

- At clump scales, the SCUBA-2 $850 \mu$ m flux measurements tend to be above the SED fitted to the shorter wavelength SPIRE bands. The effect on the $\beta$ values in the joint fits is $\Delta \beta \sim 0.2$. The result is not entirely robust with respect to the decisions made in the data reduction.

- The positions of the SCUBA-2 submillimetre clumps are positively correlated with Class I and II YSO candidates. For Class III sources, the correlation is negative.

- Compared to starless clumps, protostellar clumps have a $1-2 \mathrm{~K}$ higher median colour temperature and marginally lower $\beta$ values. The two populations mostly overlap, however. This also applies to the mass and volume density distributions.

- Most of the observed $T-\beta$ anticorrelation may be caused by noise. Evidence for the anticorrelation is found mainly at large scales.

Acknowledgements. The James Clerk Maxwell Telescope is operated by the East Asian Observatory on behalf of The National Astronomical Observatory of Japan, Academia Sinica Institute of Astronomy and Astrophysics, the Korea Astronomy and Space Science Institute, the National Astronomical Observatories of PR China and the Chinese Academy of Sciences (Grant No. XDB09000000), with additional funding support from the Science and Technology Facilities Council of the United Kingdom and participating universities in the United Kingdom and Canada. Additional funds for the construction of SCUBA-2 were provided by the Canada Foundation for Innovation. This research made use of Montage, funded by the National Aeronautics and Space Administration's Earth Science Technology Office, Computational Technologies Project, under Cooperative Agreement Number NCC5-626 between NASA and the California Institute of Technology. The code is maintained by the NASA/IPAC Infrared Science Archive. The data presented in this paper are partly based on the ESO-ARO programme ID 196.C-0999(A). M. Juvela and V.-M. Pelkonen acknowledge the support of the Academy of Finland Grant No. 285769. T. Liu is supported by KASI fellowship and EACOA fellowship. J. Malinen acknowledges the support of ERC-2015-STG No. 679852 RADFEEDBACK. V.M. Pelkonen acknowledges the financial support from the European Research Council, Advanced Grant No. 320773 entitled Scattering and Absorption of Electromagnetic Waves in Particulate Media (SAEMPL).

\section{References}

André, P., Men'shchikov, A., Bontemps, S., et al. 2010, A\&A, 518, L102 Balog, Z., Müller, T., Nielbock, M., et al. 2014, Exp. Astron., 37, 129 Barnard, E. E., Frost, E. B., \& Calvert, M. R. 1927, A Photographic Atlas of Selected Regions of the Milky Way (Carnegie Institution of Washington) Beckwith, S. V. W., Sargent, A. I., Chini, R. S., \& Guesten, R. 1990, AJ, 99, 924 Bendo, G. J., Griffin, M. J., Bock, J. J., et al. 2013, MNRAS, 433, 3062 Berry, D. S. 2015, Astron. Comput., 10, 22

Bintley, D., Holland, W. S., MacIntosh, M. J., et al. 2014, in Millimeter, Submillimeter, and Far-Infrared Detectors and Instrumentation for Astronomy VII, Proc. SPIE, 9153, 915303

Boudet, N., Mutschke, H., Nayral, C., et al. 2005, ApJ, 633, 272

Bracco, A., Palmeirim, P., André, P., et al. 2017, A\&A, 604, A52

Chapin, E. L., Berry, D. S., Gibb, A. G., et al. 2013, MNRAS, 430, 2545 Chen, M. C.-Y., Di Francesco, J., Johnstone, D., et al. 2016 ApJ, 826, 95 
Clemens, D. P. \& Barvainis, R. 1988, ApJS, 68, 257

Compiègne, M., Verstraete, L., Jones, A., et al. 2011, A\&A, 525, A103

Coupeaud, A., Demyk, K., Meny, C., et al. 2011, A\&A, 535, A124

Demyk, K., Meny, C., Lu, X.-H., et al. 2017, A\&A, 600, A123

Désert, F., Macías-Pérez, J. F., Mayet, F., et al. 2008, A\&A, 481, 411

Drabek, E., Hatchell, J., Friberg, P., et al. 2012, MNRAS, 426, 23

Dupac, X., Bernard, J., Boudet, N., et al. 2003, A\&A, 404, L11

Dutra, C. M. \& Bica, E. 2002, A\&A, 383, 631

Etxaluze, M., Smith, H. A., Tolls, V., Stark, A. A., \& González-Alfonso, E. 2011, AJ, 142, 134

Friesen, R. K., Johnstone, D., Naylor, D. A., \& Davis, G. R. 2005, MNRAS, 361, 460

Galametz, M., Kennicutt, R. C., Albrecht, M., et al. 2012, MNRAS, 425, 763

Griffin, M. J., Abergel, A., Abreu, A., et al. 2010, A\&A, 518, L3

Hildebrand, R. H. 1983, QJRAS, 24, 267

Holland, W. S., Bintley, D., Chapin, E. L., et al. 2013, MNRAS, 430, 2513

Jenness, T., Berry, D., Chapin, E., et al. 2011, in Astronomical Data Analysis Software and Systems XX, eds. I. N. Evans, A. Accomazzi, D. J. Mink, A. H. Rots, ASP Conf. Ser., 442, 281

Juvela, M. \& Ysard, N. 2012a, A\&A, 541, A33

Juvela, M. \& Ysard, N. 2012b, A\&A, 539, A71

Juvela, M., Ristorcelli, I., Montier, L. A., et al. 2010, A\&A, 518, L93

Juvela, M., Ristorcelli, I., Pagani, L., et al. 2012, A\&A, 541, A12

Juvela, M., Montillaud, J., Ysard, N., \& Lunttila, T. 2013, A\&A, 556, A63

Juvela, M., Demyk, K., Doi, Y., et al. 2015a, A\&A, 584, A94

Juvela, M., Ristorcelli, I., Marshall, D. J., et al. 2015b, A\&A, 584, A93

Kim, J., Lee, J.-E., Liu, T., et al. 2017, ApJS, 231, 9

Li, A. \& Draine, B. T. 2001, ApJ, 554, 778

Li, J. I., Liu, H. B., Hasegawa, Y., \& Hirano, N. 2017, ApJ, 840, 72

Liu, T., Zhang, Q., Kim, K.-T., et al. 2016, ApJS, 222, 7

Liu, T., Kim, K.-T., Juvela, M., et al. 2018, ApJS, 234, 28

Lombardi, M., Bouy, H., Alves, J., \& Lada, C. J. 2014, A\&A, 566, A45

Lynds, B. T. 1962, ApJS, 7, 1

Magnani, L., Blitz, L., \& Mundy, L. 1985, ApJ, 295, 402

Mairs, S., Johnstone, D., Kirk, H., et al. 2016, MNRAS, 461, 4022

Malinen, J., Juvela, M., Collins, D. C., Lunttila, T., \& Padoan, P. 2011, A\&A 530, A101

Malinen, J., Juvela, M., Zahorecz, S., et al. 2014, A\&A, 563, A125

Malinen, J., Montier, L., Montillaud, J., et al. 2016, MNRAS, 460, 1934

Marshall, D. J., Robin, A. C., Reylé, C., Schultheis, M., \& Picaud, S. 2006, A\&A, 453, 635

Marshall, D. J., Joncas, G., \& Jones, A. P. 2009, ApJ, 706, 727

Martin, P. G., Roy, A., Bontemps, S., et al. 2012, ApJ, 751, 28

Marton, G., Tóth, L. V., Paladini, R., et al. 2016, MNRAS, 458, 3479

Meng, F., Wu, Y., \& Liu, T. 2013, ApJS, 209, 37

Miville-Deschênes, M. \& Lagache, G. 2005, ApJS, 157, 302

Montier, L. A., Pelkonen, V.-M., Juvela, M., Ristorcelli, I., \& Marshall, D. J. 2010, A\&A, 522, A83

Montillaud, J., Juvela, M., Rivera-Ingraham, A., et al. 2015, A\&A, 584, A92

Moore, T. J. T., Plume, R., Thompson, M. A., et al. 2015, MNRAS, 453, 4264

Myers, P. C., Linke, R. A., \& Benson, P. J. 1983, ApJ, 264, 517

Pagani, L., Lefèvre, C., Juvela, M., Pelkonen, V.-M., \& Schuller, F. 2015, A\&A, 574, L5

Paradis, D., Veneziani, M., Noriega-Crespo, A., et al. 2010, A\&A, 520, L8

Paradis, D., Paladini, R., Noriega-Crespo, A., et al. 2012, A\&A, 537, A113

Pattle, K., Ward-Thompson, D., Kirk, J. M., et al. 2015, MNRAS, 450, 1094

Pattle, K., Ward-Thompson, D., Kirk, J. M., et al. 2017, MNRAS, 464, 4255

Piazzo, L., Calzoletti, L., Faustini, F., et al. 2015, MNRAS, 447, 1471

Pilbratt, G. L., Riedinger, J. R., Passvogel, T., et al. 2010, A\&A, 518, L1

Planck Collaboration XVII. 2011, A\&A, 536, A17

Planck Collaboration XIX. 2011, A\&A, 536, A19

Planck Collaboration XXII. 2011, A\&A, 536, A22

Planck Collaboration XXIII. 2011, A\&A, 536, A23

Planck Collaboration XXV. 2011, A\&A, 536, A25

Planck Collaboration XI. 2014, A\&A, 571, A11

Planck Collaboration Int. XIV. 2014, A\&A, 564, A45

Planck Collaboration Int. XVII. 2014, A\&A, 566, A55

Planck Collaboration I. 2016, A\&A, 594, A1

Planck Collaboration X. 2016, A\&A, 594, A10

Planck Collaboration VIII. 2016, A\&A, 594, A8

Planck Collaboration XXVIII. 2016, A\&A, 594, A28

Poglitsch, A., Waelkens, C., Geis, N., et al. 2010, A\&A, 518, L2

Rivera-Ingraham, A., Ristorcelli, I., Juvela, M., et al. 2016, A\&A, 591, A90

Rivera-Ingraham, A., Ristorcelli, I., Juvela, M., et al. 2017, A\&A, 601, A94

Robin, A., Reylé, C., Derrière, S., \& Picaud, S. 2003, A\&A, 409, 523
Rodón, J. A., Zavagno, A., Baluteau, J., et al. 2010, A\&A, 518, L80

Roy, A., Martin, P. G., Polychroni, D., et al. 2013, ApJ, 763, 55

Sadavoy, S. I., Di Francesco, J., Johnstone, D., et al. 2013, ApJ, 767, 126

Sadavoy, S. I., Stutz, A. M., Schnee, S., et al. 2016, A\&A, 588, A30

Schnee, S., Enoch, M., Noriega-Crespo, A., et al. 2010, ApJ, 708, 127

Shetty, R., Kauffmann, J., Schnee, S., \& Goodman, A. A. 2009a, ApJ, 696, 676

Shetty, R., Kauffmann, J., Schnee, S., Goodman, A. A., \& Ercolano, B. 2009b, ApJ, 696, 2234

Shirley, Y. L., Nordhaus, M. K., Grcevich, J. M., et al. 2005, ApJ, 632, 982

Skrutskie, M., Cutri, R., Stiening, R., et al. 2006, AJ, 131, 1163

Tatematsu, K., Liu, T., Ohashi, S., et al. 2017, ApJS, 228, 12

Tauber, J. A., Mandolesi, N., Puget, J., et al. 2010, A\&A, 520, A1

Veneziani, M., Ade, P. A. R., Bock, J. J., et al. 2010, ApJ, 713, 959

Wang, K., Zahorecz, S., Cunningham, M. R., et al. 2018, Res. Notes AAS, 2, 2 , http://stacks.iop.org/2515-5172/2/i=1/a

Ward-Thompson, D., Pattle, K., Kirk, J. M., et al. 2016, MNRAS, 463, 1008

Wright, E. L., Eisenhardt, P. R. M., Mainzer, A. K., et al. 2010, AJ, 140, 1868

Ysard, N., Juvela, M., Demyk, K., et al. 2012, A\&A, 542, A21

Zhang, T., Wu, Y., Liu, T., \& Meng, F. 2016, ApJS, 224, 43

1 Department of Physics, University of Helsinki, PO Box 64, 00014, Finland,

e-mail: mika . juvela@helsinki . fi

2 Yunnan Observatories, Chinese Academy of Sciences, 396 Yangfangwang, Guandu District, Kunming 650216, PR China

3 Chinese Academy of Sciences South America Center for Astronomy, China-Chile Joint Center for Astronomy, Camino El Observatorio 1515, Las Condes, Santiago, Chile

4 Key Laboratory for the Structure and Evolution of Celestial Objects, Chinese Academy of Sciences, 396 Yangfangwang, Guandu District, Kunming 650216, PR China

5 Center for Astronomical Mega-Science, Chinese Academy of Sciences, 20A Datun Road, Chaoyang District, Beijing 100012, PR China

6 Jeremiah Horrocks Institute, University of Central Lancashire, Preston PR1 2HE, UK

7 Korea Astronomy and Space Science Institute, 776 Daedeokdaero, Yuseong-gu, Daejeon 34055, Republic of Korea

8 East Asian Observatory, 660 N. A'ohōkū Place, Hilo, Hawaii 96720 2700, USA

9 UK ALMA Regional Centre Node, Jodrell Bank Centre for Astrophysics, The University of Manchester, Oxford Road, Manchester M13 9PL, UK

10 Astrophysics Research Institute, Liverpool John Moores University, Ic2, Liverpool Science Park, 146 Brownlow Hill, Liverpool, L3 5RF, $\mathrm{UK}$

11 Konkoly Observatory, Research Centre for Astronomy and Earth Sciences, Hungarian Academy of Sciences, 1121 Budapest, Konkoly Thege Miklós út 15-17, Hungary

12 Eövös Loránd University, Department of Astronomy, Pázmány Péter sétány 1/A, 1117 Budapest, Hungary

13 Department of Physics and Astronomy, University of Waterloo, Waterloo, Ontario N2L 3G1, Canada

14 Institute of Astronomy and Astrophysics, Academia Sinica, 11F of Astronomy-Mathematics Building, AS/NTU No.1, Sec. 4, Roosevelt Rd, Taipei 10617, Taiwan

15 National Astronomical Observatories, Chinese Academy of Sciences, Beijing 100012, PR China

16 Key Laboratory of Radio Astronomy, Chinese Academy of Science, Nanjing 210008, PR China

17 Institute of Physics I, University of Cologne, Germany

18 Laboratoire AIM, IRFU/Service d'Astrophysique - CEA/DSM CNRS - Université Paris Diderot, Bât. 709, CEA-Saclay, 91191, Gif-sur-Yvette Cedex, France

19 Université de Toulouse, UPS-OMP, IRAP, 31028 Toulouse cedex 4, France

20 CNRS, IRAP, 9 av. colonel Roche, BP 44346, 31028 Toulouse cedex 4, France 
${ }^{21}$ Graduate Institute of Astronomy, National Central University 300, Jhongli , Taoyuan 32001, Taiwan

22 Nobeyama Radio Observatory, National Astronomical Observatory of Japan, National Institutes of Natural Sciences, 462-2 Nobeyama, Minamimaki, Minamisaku, Nagano 384-1305, Japan

${ }^{23}$ Centre for Astrophysics Research, School of Physics Astronomy \& Mathematics, University of Hertfordshire, College Lane, Hatfield AL10 9AB, UK

24 IAPS - INAF, via Fosso del Cavaliere, 100, 00133 Roma, Italy
${ }^{25}$ European Southern Observatory, Karl-Schwarzschild-Str.2, 85748 Garching bei München, Germany

${ }^{26}$ Department of Astronomy, Peking University, 100871 Beijing, PR China

27 School of Space Research, Kyung Hee University, 1732, Deogyeongdaero, Giheung-gu, Yongin-si, Gyeonggi-do, Republic of Korea

28 Department of Astronomy and Space Science, Chungnam National University, 99 Daehak-ro, Yuseong-gu, Daejeon 34134, Korea 


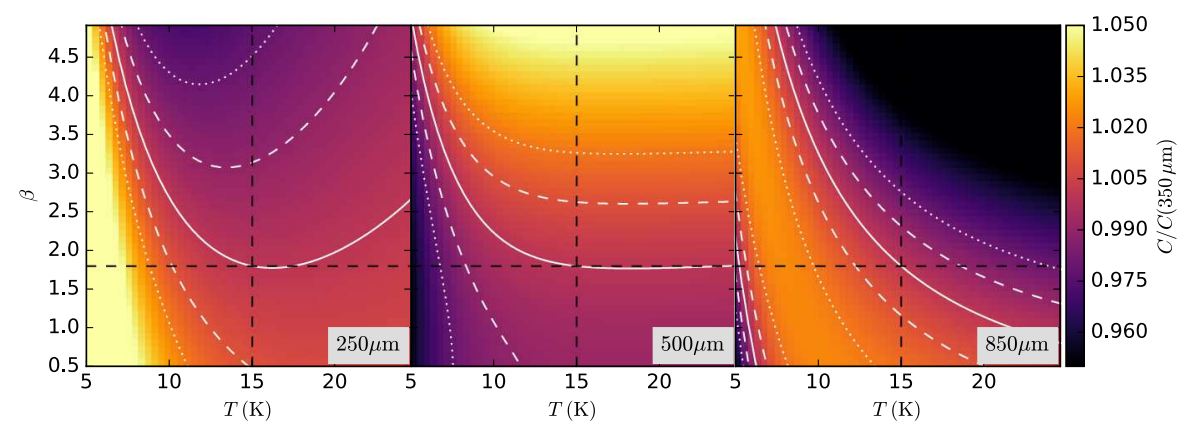

Fig. A.1. Colour correction factors at the $250 \mu \mathrm{m}, 500 \mu \mathrm{m}$, and $850 \mu \mathrm{m}$ bands relative to the same at $350 \mu \mathrm{m}$. The ratios are normalised to one at $T=15 \mathrm{~K}$ and $\beta=1.5$. The contours denote the ratio one (solid lines) and deviations from one by $\pm 1 \%$ (dashed lines) and $\pm 2 \%$ (dotted lines).

\section{Appendix A: Colour corrections}

The maps were colour-corrected pixel by pixel, using the MBB fits $B(T) \times v^{1.8}$ to the original, unfiltered SPIRE data. Because the fits that include the $850 \mu \mathrm{m}$ point can lead to different SEDs, we examined how the colour correction factors vary over the plausible parameter range. Figure A.1 compares the ratios of colour correction factors ${ }^{8}$ relative to these factors at $350 \mu \mathrm{m}$. The normalisation with the $350 \mu \mathrm{m}$ values is included because we are interested only in the SED shape, not its normalisation. In the plot, the ratio is further normalised so that it is one for $T=15.0 \mathrm{~K}$ and $\beta=1.5$. If the ratios significantly change between the position where colour correction was estimated and the true SED shape, it would bias the fit results.

According to Fig. A.1, the relative variations are of about $2 \%$ or smaller over most of the plausible parameter space. Since the differences between the fitted and the unknown true SED shape should be smaller, and if the differences are caused by noise, they are mostly along the line between the high $T$ and low $\beta$ and the low $T$ and high $\beta$ combinations. Thus, the colour correction uncertainties are likely to be at most $\sim 2 \%$ and thus remain much smaller than the typical photometric errors, which are closer to $10 \%$. A $2 \%$ relative error between $350 \mu \mathrm{m}$ and $850 \mu \mathrm{m}$ corresponds to a spectral index error smaller than $\Delta \beta=0.05$.

\section{Appendix B: CO contamination in the $850 \mu \mathrm{m}$ data}

We do not have high-resolution observations of the CO $J=3-2$ lines to directly correct the SCUBA-2 $850 \mu \mathrm{m}$ data for line contamination, but we can use lower transition data to estimate the possible effect.

The ${ }^{12} \mathrm{CO}(1-0)$ maps of Meng et al. (2013) and Zhang et al. (2016) cover a number of SCUBA-2 targets. The CO observations were made with the Purple Mountain Observatory telescope, which at $115 \mathrm{GHz}$ has a beam size of $52^{\prime \prime}$. This is sufficiently close to $40^{\prime \prime}$, the resolution of the maps where flux densities were measured, so that a direct comparison is meaningful. The ratio between the $J=3-2$ and $J=1-0$ lines is not known. The line ratios were discussed in Juvela et al. (2015a), where they were found to be mostly $\sim 0.3$ or below, both based on Planck measurements of the velocity-integrated CO emission and based on examples of ground-based measurements. The low line ratios are explained by the nature of our sources, PGCC being a selection of the coldest dust emission sources on the sky. This does not mean that the line ratios could not be much higher

8 Colour correction factor is here defined such that original data divided by the factor results in an estimate of the monochromatic value at the reference wavelength.

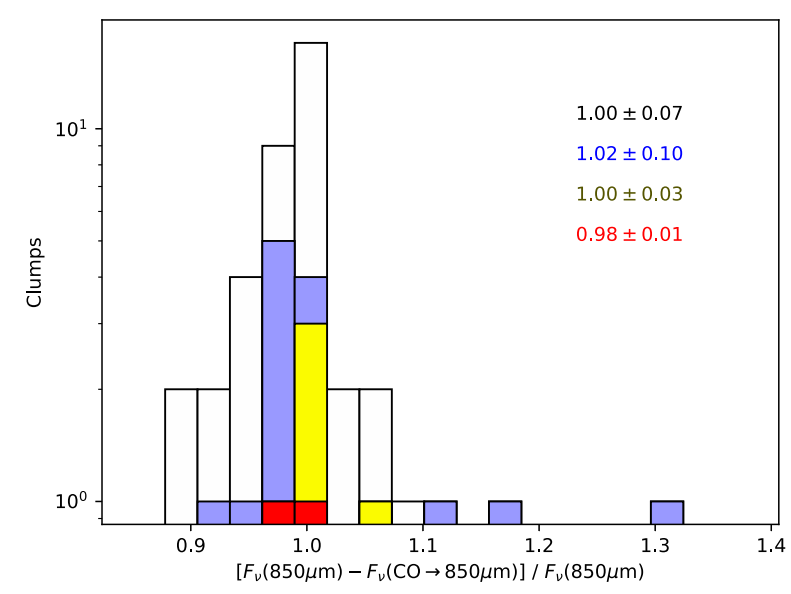

Fig. B.1. Ratio of CO-corrected and original $850 \mu \mathrm{m}$ flux values. The values are for a subset of clumps from the $P\left(\chi^{2}\right) 85 \%, 25 \%, 10 \%$, and $5 \%$ samples (white, blue, yellow, and red, respectively) with the LM data version. The mean and standard deviation values are listed in the figure.

in individual, actively star-forming clumps. In the following we assume a line ratio of one, which thus very likely overestimates the typical CO contamination. For example, Fig. B.1 suggested that the line ratios $(2-1) /(1-0)$ would be lower than 0.5 .

We scaled the CO emission to $850 \mu \mathrm{m}$ flux density with a constant $0.70 \mathrm{mJy}_{\text {beam }}^{-1}$ per $\mathrm{K} \mathrm{km} \mathrm{s}^{-1}$ (Drabek et al. 2012), and similar to continuum photometry, calculated the difference between clump apertures and reference annuli. The exercise was made using the LM data version. We did not process the CO maps through the SCUBA pipeline. This should lead to an overestimation of the $\mathrm{CO}$ effects and not to an underestimation because we ignored the loss of CO intensity that is caused by the spatial filtering.

Figure B.1 shows the ratios between $\mathrm{CO}$-corrected and original $850 \mu \mathrm{m}$ flux densities. The mean value is close to one, and for many clumps, the CO correction would increase the $850 \mu \mathrm{m}$ flux density estimates. This means that the $\mathrm{CO}$ correction was larger outside the clump, which is not expected given that the cold clumps are not strong CO emitters. Molecular depletion and the anticorrelation with local heating sources can both lead to a situation where the $\mathrm{CO}$ emission is stronger outside the cold clump.

Figure B.1 suggests that even assuming a line ratio (3-2)/(10 ) of one, the effect of the CO contamination on the $850 \mu \mathrm{m}$ flux density would typically be about two percent. Even if the $\mathrm{CO}$ correction were a systematic $-5 \%$ for most clumps, the net 
effect on the median $\beta$ of the $250-850 \mu$ m fits would be only $\Delta \beta=+0.10$.

\section{Appendix C: Plots of Herschel and SCUBA-2 maps}

Figure C.1 shows maps of $250 \mu \mathrm{m}$ optical depth and $850 \mu \mathrm{m}$ surface brightness for all the 96 fields.

The optical depths $\tau(250 \mu \mathrm{m})$ are based on MBB fits of the SPIRE $250 \mu \mathrm{m}, 350 \mu \mathrm{m}$, and $500 \mu \mathrm{m}$ data, without background subtraction. The intensity zero-points of the input maps (obtained from HSA) were set through a comparison with Planck data. For optical depths, our SED fits use a constant value of $\beta=1.8$ and a map resolution of $40^{\prime \prime}$. The SCUBA-2 $850 \mu \mathrm{m}$ maps correspond the LM version of data. (see Sect. 2.1). These are shown at the full resolution of $14^{\prime \prime}$ and are scaled to surface brightness units $\mathrm{MJy} \mathrm{sr}^{-1}$.

The contours in the $\tau(250 \mu \mathrm{m})$ maps indicate the extent of the LM and M850 source masks that were used in the SCUBA-2 pipeline. The clumps extracted with the Fellwalker method are indicated in the $850 \mu \mathrm{m}$ maps with white contours. Only the sources with dashed black contours were used in investigations of the spectral index values.

\section{Appendix D: SED fits of Planck data alone}

In Sect. 4.1.1 the mean emission of the fields was fitted with IRAS and Planck data. The shortest Planck wavelength of $350 \mu \mathrm{m}$ is near the maximum of the cold dust emission spectrum. Nevertheless, we can attempt SED fits also without the IRAS data point (Fig. D.1).

Although the scatter is significantly larger than in Fig. 1, the median values are similar to the fits where the IRAS $100 \mu \mathrm{m}$ point was also used. There is no evidence that the latter would be significantly affected by very small grain emission or by temperature variations inside the measurement aperture. As in Fig. 1a, the extension of the fitted wavelength range to $1380 \mu \mathrm{m}$ slightly decreases the median $\beta$ value.

\section{Appendix E: Dependence between dust parameters and error estimates}

The dependence of $\beta$ estimated from SPIRE and SCUBA- 2 data is compared to some other parameters in Fig. E.1. The estimates are almost independent of the (non-reduced) $\chi^{2}$ values of the fits and are weakly correlated with the colour temperature that is estimated from $250-500 \mu \mathrm{m}$ data using a fixed value of $\beta=1.8$. Finally, Fig. E.1c shows some negative correlation with the source brightness. However, none of the correlations are statistically significant.

It would seem natural to analyse $\beta$ distributions using the sources with the lowest $\beta$ error estimates. However, the $(T, \beta)$ anticorrelation leads to a narrow, curved $\chi^{2}$ valley in the $(T$, $\beta$ ) plane. In the case of large uncertainties, it runs at low $T$ runs parallel to the $\beta$ axis and at high $T$ parallel to temperature axis. Thus, the selection of sources with a low $\beta$ uncertainty would give preference to warm sources with low $\beta$ values. The effect is illustrated in Fig. E.2, where the error estimates $\delta(T)$ and $\delta(\beta)$ correspond to half of the interquartile range estimated from MCMC runs. As expected, the figure shows a correlation between $\beta$ and the error estimate of the $\beta$ uncertainty. Subsamples selected based on the $\chi^{2}$ values of the fits are not significantly correlated with $\delta(T)$ or $\delta(\beta)$.

\section{Appendix F: Effect of map making and clump selection on $\beta$}

The obtained $\beta$ distributions depend on our decisions regarding the map making and the data calibration. In map making, the options were to use $200^{\prime \prime}$ or $500^{\prime \prime}$ filtering scale. Furthermore, maps could be produced using either large or small source masks based on Herschel $500 \mu \mathrm{m}$ data (SM and LM, respectively) or masks based on SCUBA-2 $850 \mu \mathrm{m}$ data themselves (M850). For illustration of the calibration uncertainty of the $850 \mu \mathrm{m}$ data, we compared the result for the flux conversion factor $\mathrm{FCF}=2.41 \mathrm{Jy}(\mathrm{pW})^{-1}$, estimated from calibration measurements near the observing times, to the results obtained with the default calibration value of $\mathrm{FCF}=2.34 \mathrm{Jy}(\mathrm{pW})^{-1} \operatorname{arcsec}^{-2}$.

The following table describes the $\beta$ distributions. These include the $\beta$ distributions calculated using SPIRE data alone (unfiltered and filtered versions) because the clump selection also changes from case to case.

The data show that the two cases of the $850 \mu \mathrm{m}$ calibration differ by less than $\Delta \beta=0.1$, except for the M850 case, where the larger FCF factor results in a median spectral index value lower by 0.2 units.

\section{Appendix G: Effect of noise on the $\beta(T)$ relation}

In Sect. 5.4 we used simulations to test the significance of the observed negative correlation between the colour temperature and the spectral index. The noise was kept as a free parameter because otherwise the result would critically depend on the precision of the a priori estimates of photometric errors. Here we examine the method further with purely synthetic observations.

We assumed an intrinsic dependence of $\beta(T)=(T / 15 \mathrm{~K})^{B}$ and simulated observations at $250 \mu \mathrm{m}, 350 \mu \mathrm{m}, 500 \mu \mathrm{m}$, and $850 \mu \mathrm{m}$. The default photometric errors were $4 \%$ for the first three and $10 \%$ for the $850 \mu \mathrm{m}$ band. We generated a sample of 100 clumps. The temperatures followed a normal distribution with a mean of $15 \mathrm{~K}$ and a standard deviation of $3 \mathrm{~K}$. Together with the $\beta(T)$ relation, this defines the source fluxes, to which we added normally distributed photometric errors. The fitting of a set of synthetic observations gives the observed values of $B$ and the rms dispersion with respect to the fitted $\beta(T)$ curve.

The analysis of these synthetic observations proceeded as described in Sect. 5.4. We generated a similar sample of observations under the assumption of a flat $\beta(T)$ relation. This was obtained by fitting the syntetic observations with a fixed value of $\beta$ and replacing the flux density values by the values predicted by the fit. We then examined the $B$ parameter and the rms dispersion of $\beta$ vs. the fitted $\beta(T)$ relation of this simulated sample as a function of the noise level. The noise level was again scaled by the parameter $k$ (noise). When the rms noise in the simulation matched the rms noise of the (this time synthetic) observations, we checked whether the corresponding value of $B$ in the simulation was higher than the value of $B$ in the observations. When the difference was positive and significant, we concluded that the observations exhibited a non-constant $\beta(T)$ relation $(\beta$ decreasing with temperature) that could not be explained by the noise.

Figure G.1 shows the results for two values of $B$ and $k$ (noise). The simulations were made for 100 values between $k($ noise $)=0.1$ and $k$ (noise $)=2.0$, which thus also indicate the dispersion between different realisations. The left-hand frames correspond to cases where the observational noise is half of the default. The observed rms dispersion of $\beta$ values is matched at 
M. Juvela et al.: Herschel and SCUBA-2 observations of dust emission in a sample of Planck cold clumps
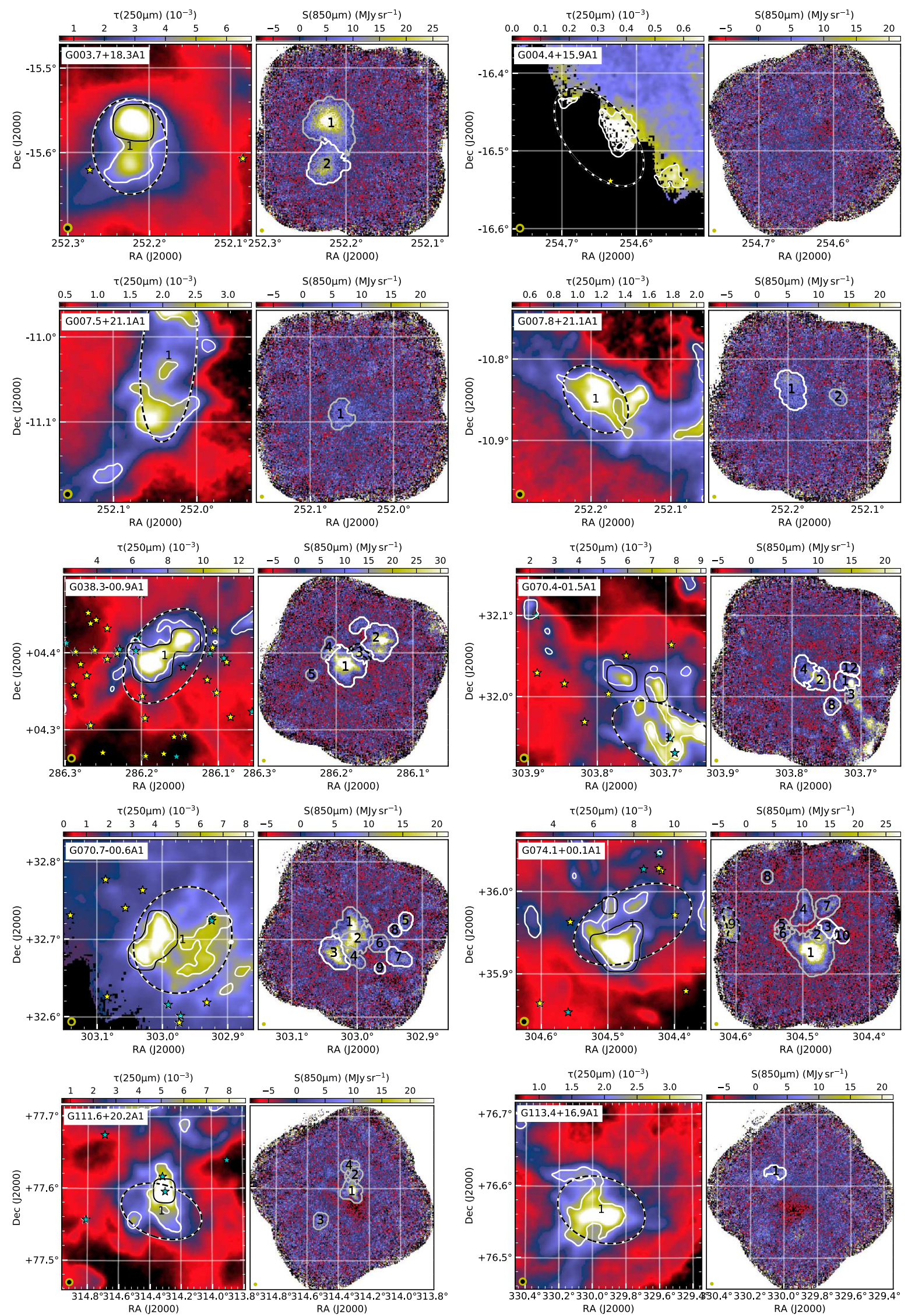

Fig. C.1. Maps of $250 \mu \mathrm{m}$ optical depth and $850 \mu \mathrm{m}$ surface brightness. In $\tau(250 \mu \mathrm{m})$ maps, white and black contours show the extent of the LM and M850 source masks, respectively. Dashed ellipses show the FWHM size of PGCC clumps (numbers at the PGCC centre coordinates refer to the numbering in Table H.3). The stars stand for the Class I-II (cyan stars) and Class III (yellow stars) YSO candidates (Marton et al. 2016). In the $S(850 \mu \mathrm{m})$ maps, the clumps of the $P\left(\chi^{2}\right)=85 \%$ sample are indicated with white contours and the other clumps with grey contours. 

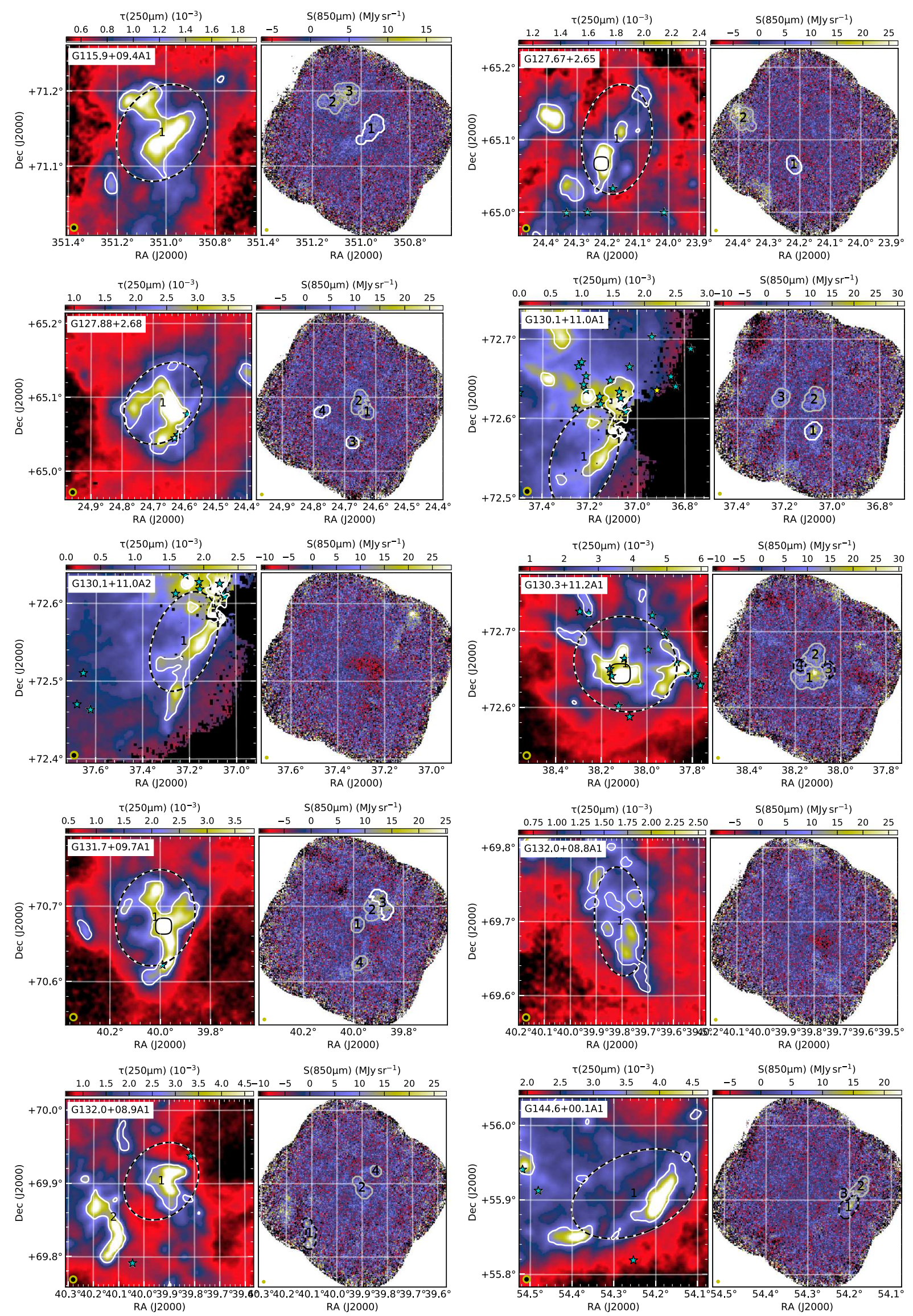

Fig. C.1. continued. 
M. Juvela et al.: Herschel and SCUBA-2 observations of dust emission in a sample of Planck cold clumps
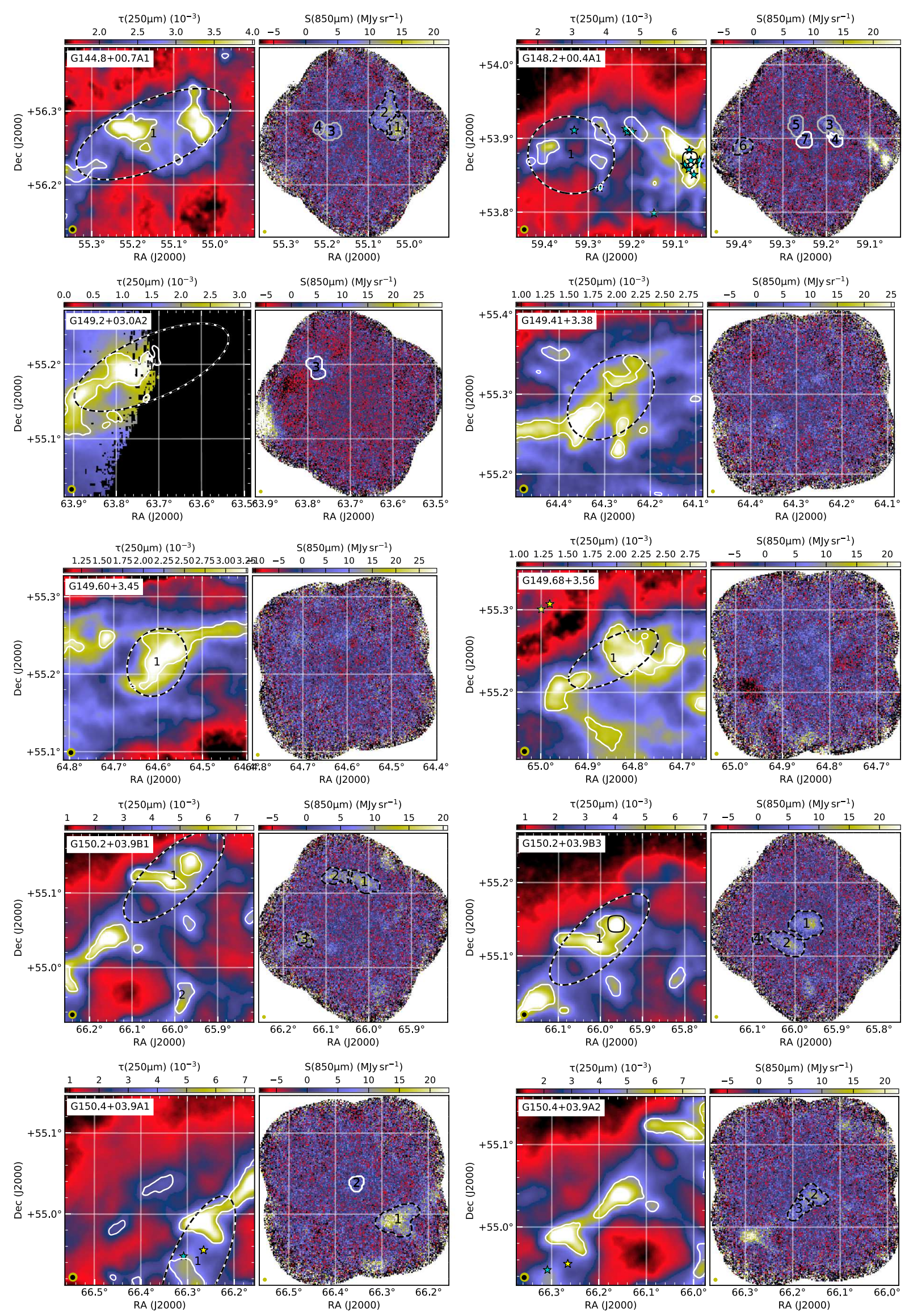

Fig. C.1. continued. 

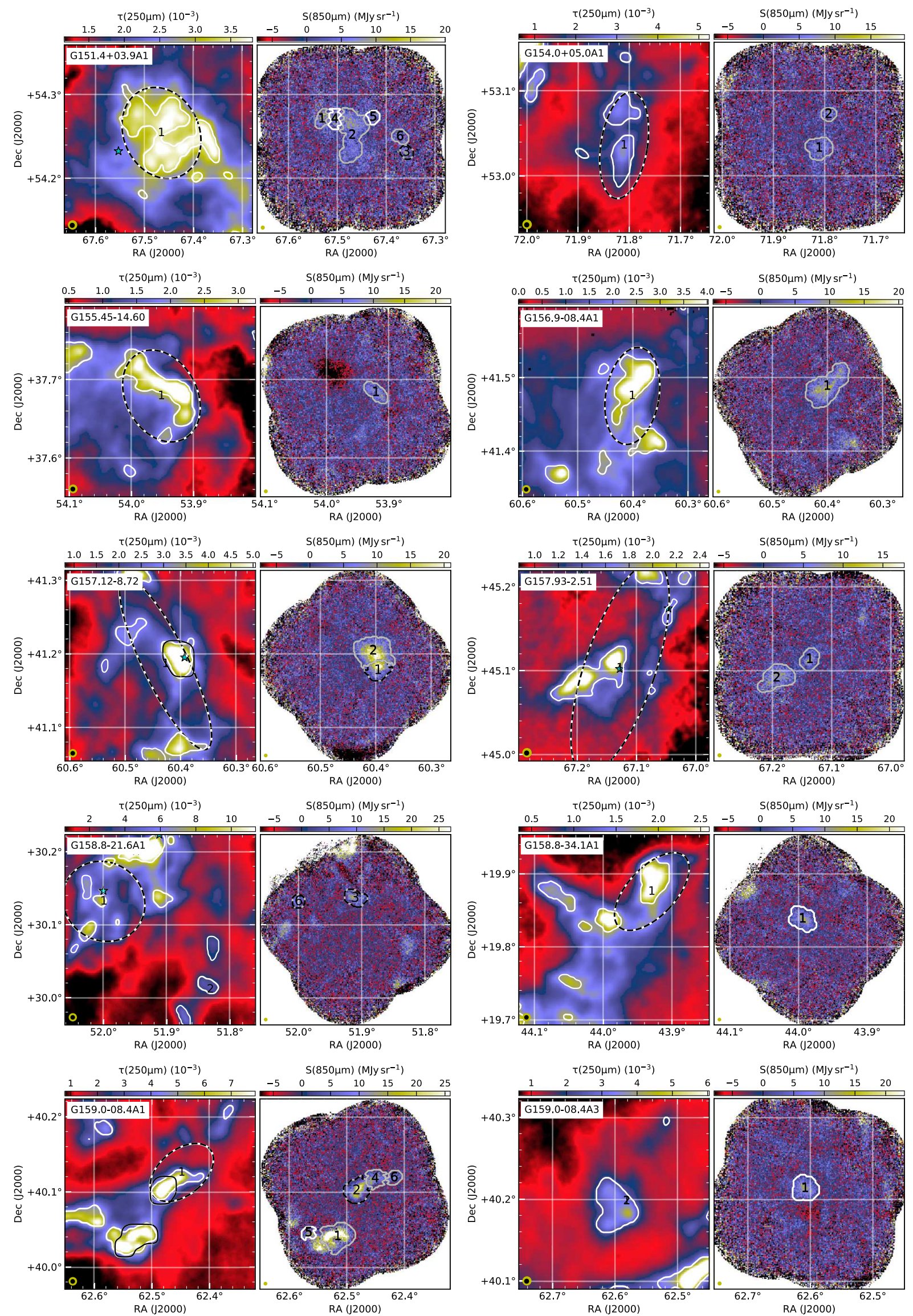

Fig. C.1. continued. 
M. Juvela et al.: Herschel and SCUBA-2 observations of dust emission in a sample of Planck cold clumps
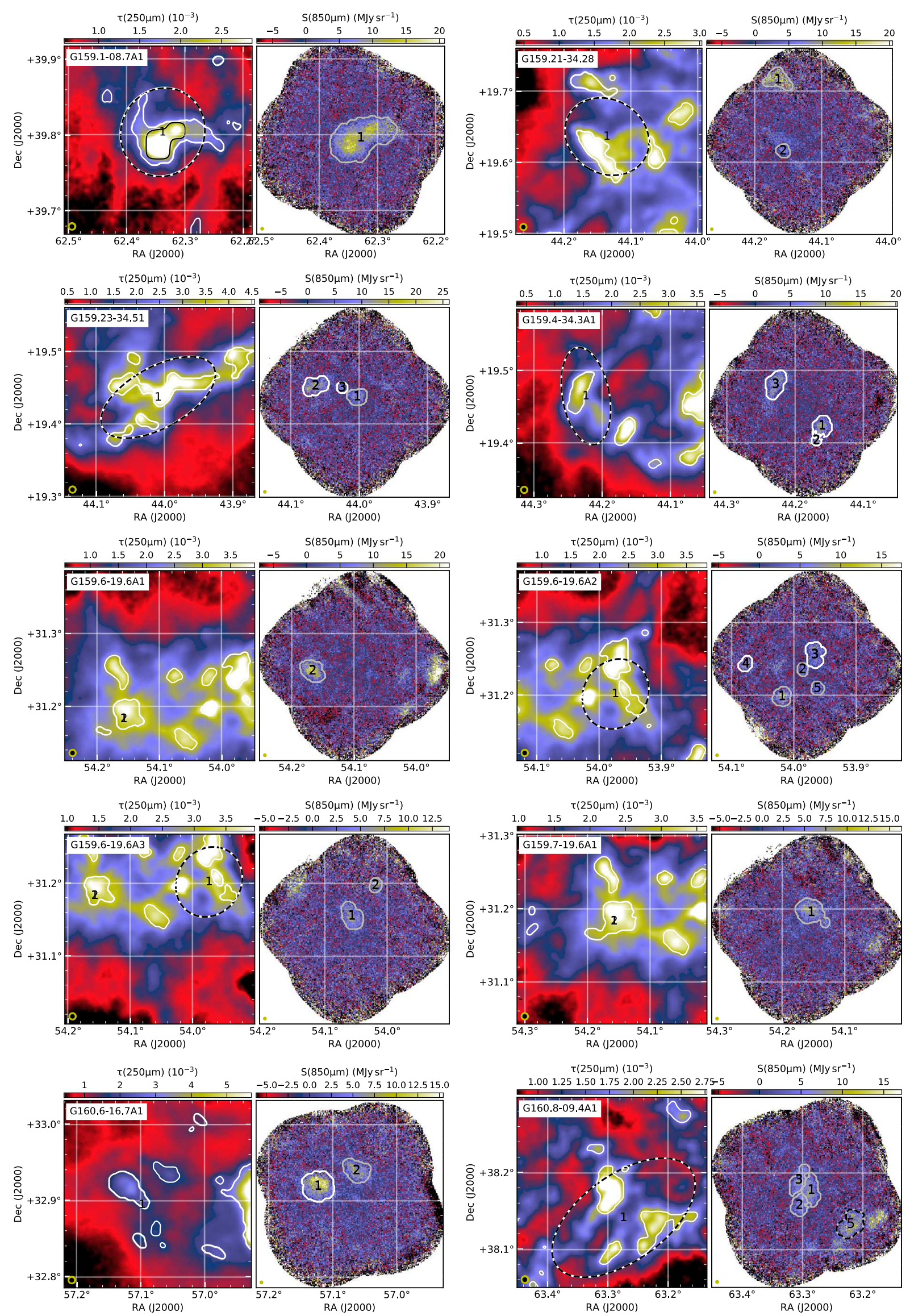

Fig. C.1. continued. 
A\&A 612, A71 (2018)
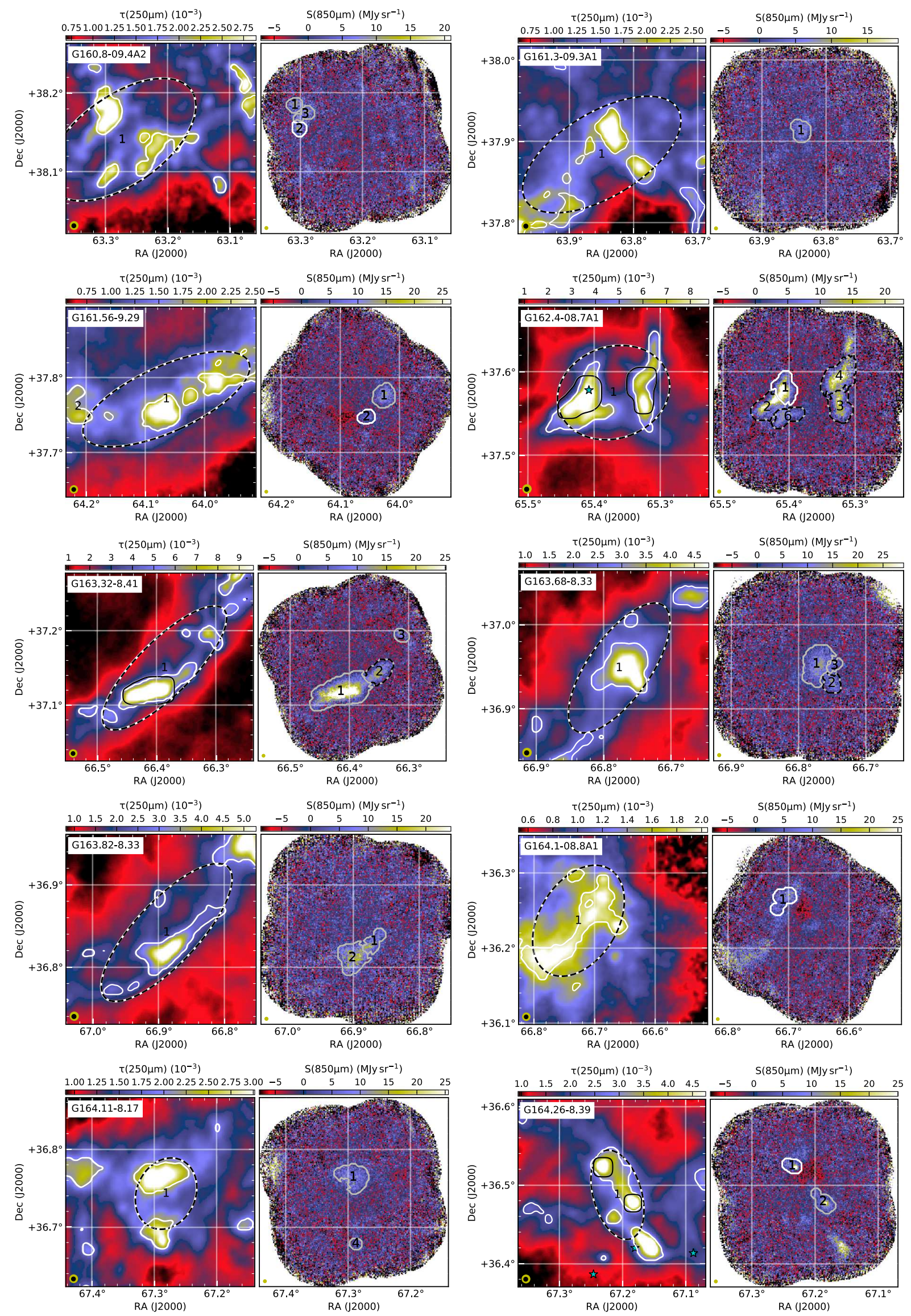

Fig. C.1. continued. 
M. Juvela et al.: Herschel and SCUBA-2 observations of dust emission in a sample of Planck cold clumps
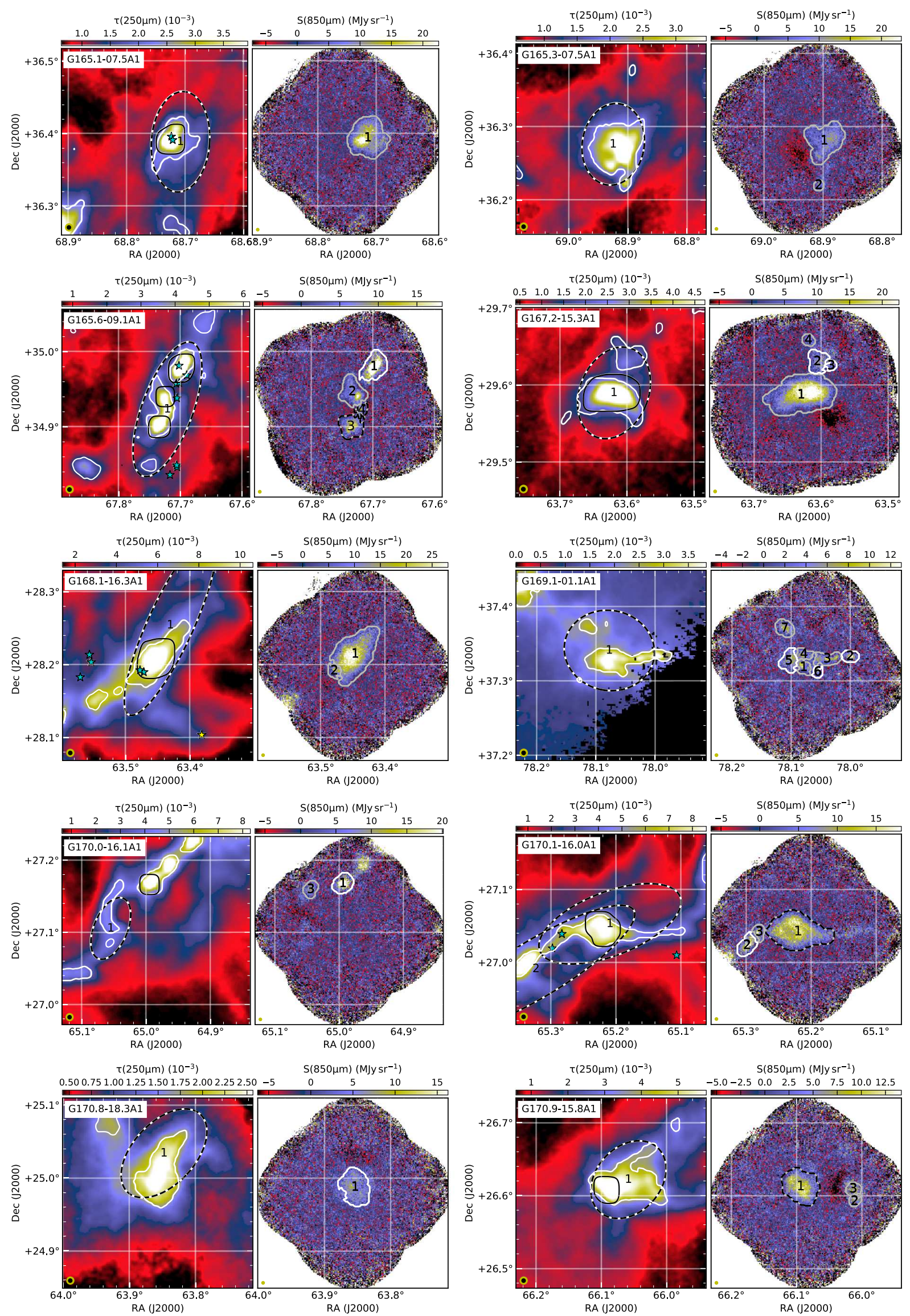

Fig. C.1. continued. 

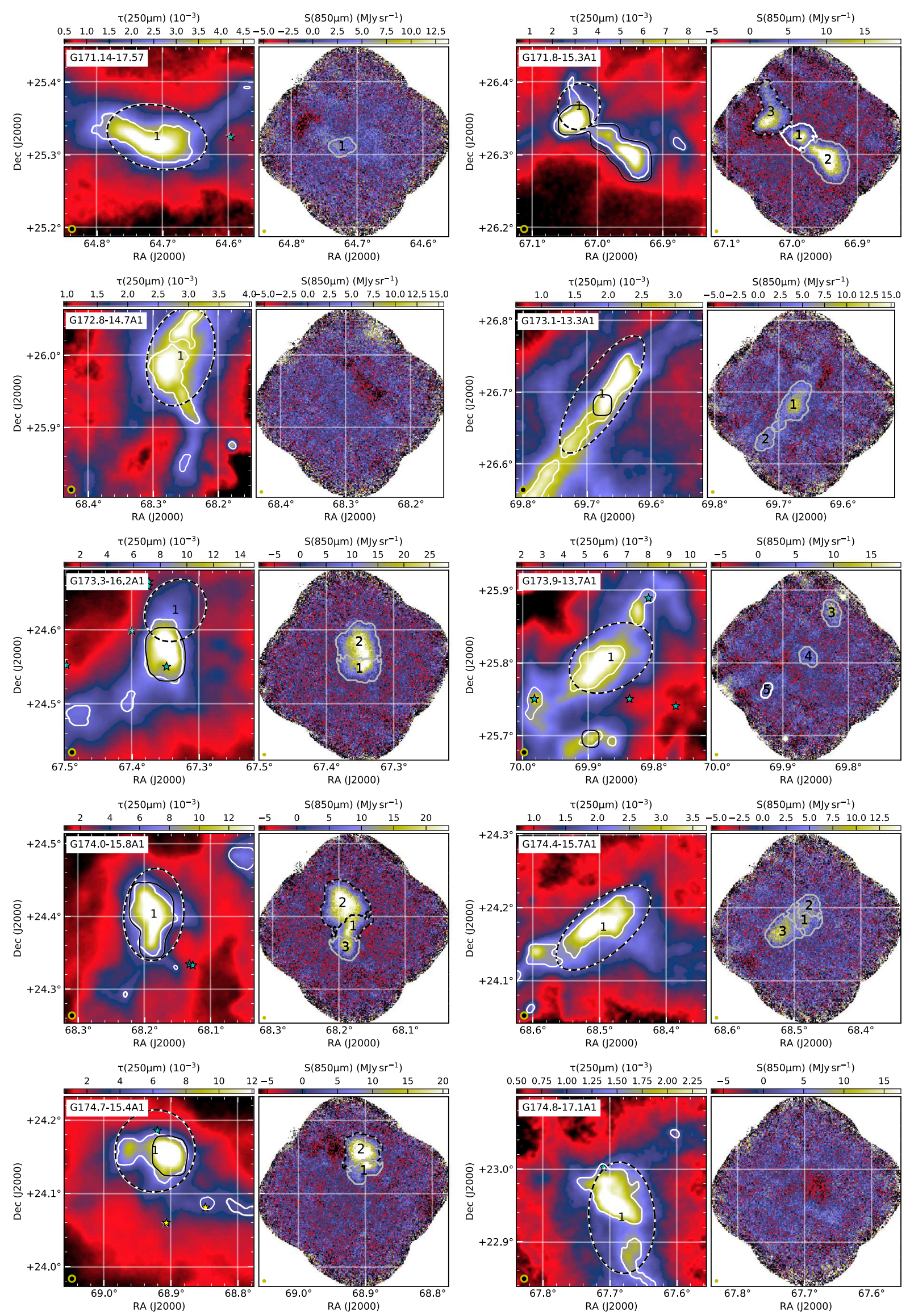

Fig. C.1. continued. 
M. Juvela et al.: Herschel and SCUBA-2 observations of dust emission in a sample of Planck cold clumps
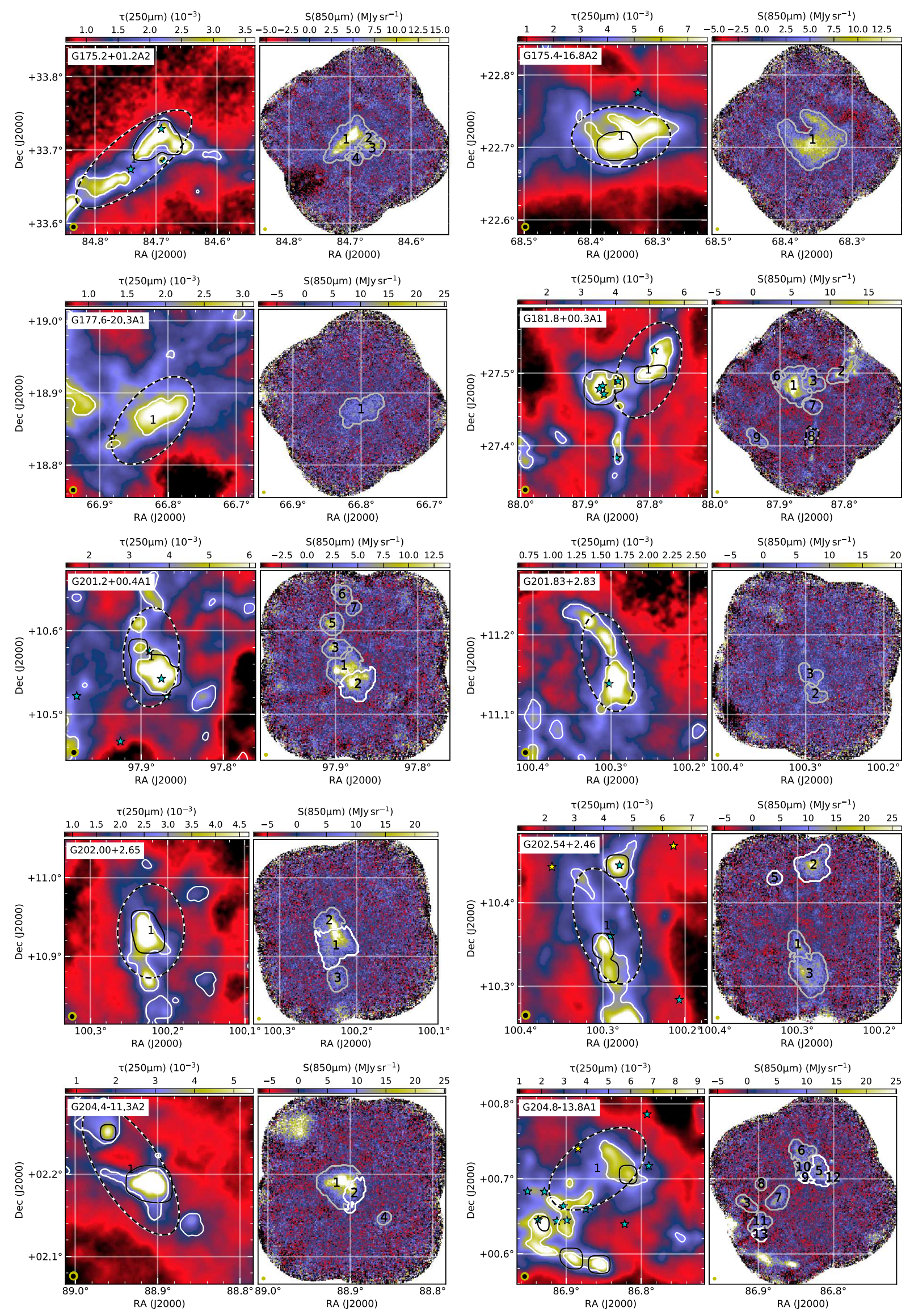

Fig. C.1. continued. 

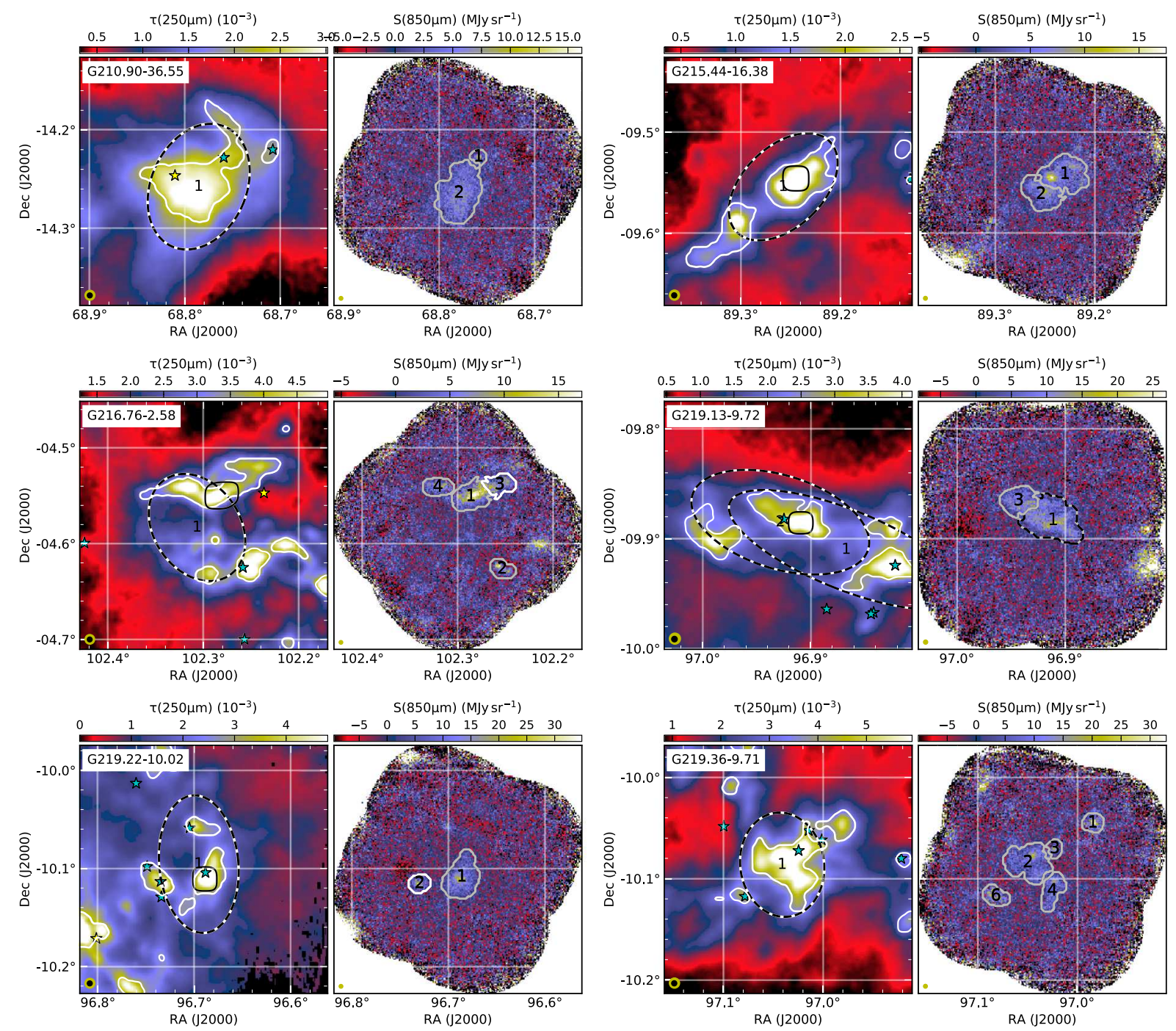

Fig. C.1. continued.

a point where the simulated $B$ is still significantly above the observed value. The test thus shows that the $T-\beta$ anticorrelation is real in the (synthetic) observations. Comparison of frames a and $\mathrm{c}$ shows that the significance of the detection (of non-constant $\beta(T)$ ) decreases as the intrinsic relation becomes flatter.

The frames $\mathrm{b}$ and $\mathrm{d}$ correspond to cases where the observational noise is $50 \%$ higher than the default values. In frame d, with $B=-0.2$, the simulations match the observed values at exactly the same $k$ (noise) value, which means that the data are compatible with a flat $\beta(T)$ model. Only when the intrinsic relation is steeper with $B=-0.6$ is the detection of the $T-\beta$ anticorrelation still found to be statistically significant (frame b). In these cases, we could proceed with further simulations to quantify the $B$ value of the intrinsic $\beta(T)$ relation. However, plots like Fig. G.1 already indicate which fraction of $B$ is caused by noise.

The recovered $k$ (noise) value (when the rms value of $\beta$ values vs. the fitted $\beta(T)$ relation matches the rms value in the

observations) is a biased estimator of the photometric errors of the observations. For example, in Fig. G.1b the difference is a factor of 1.5 between the input value $k$ (noise $)=1.5$ and the fitted value $k$ (noise) $\approx 1.0$. The effect is smaller for low absolute values of $B$ because the simulations explicitly start with the $B=0$ assumption.

Figure G.1 suggests that the precision of SPIRE and SCUBA-2 observations should be sufficient to make a detection of the anticorrelation, assuming that the default error estimates are correct and the value of $B$ is -0.2 or lower. However, the simulations are highly idealised. They do not take the possibility of systematic or non-Gaussian errors or the effects of temperature mixing into account. One of the assumptions is that all sources would follow the same $\beta(T)$ relation. In Fig. G. 2 we repeat the previous analysis, but allow a dispersion of $\sigma(\beta)=0.1$ with respect to the $\beta(T)$ relation in both the synthetic observations and in the subsequent simulations. This increases the noise in the plotted relations, but does not significantly affect the main features of Fig. G.1. 


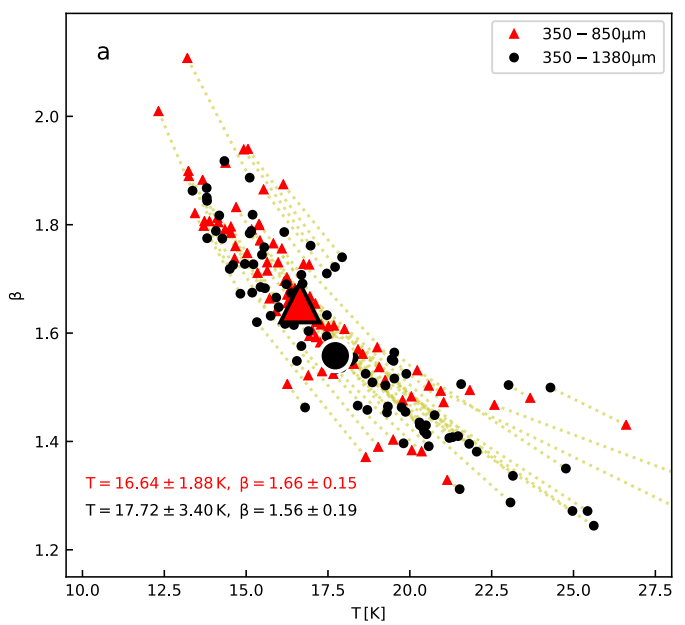

Fig. D.1. MBB fits to Planck data averaged within $8^{\prime}$ of the centre of the SCUBA-2 fields. The wavelength range is either $350-850 \mu \mathrm{m}$ or $350-1380 \mu \mathrm{m}$. Each marker corresponds to one field, and the dotted lines connect estimates of the same field. The median values are plotted with large symbols, and they are quoted in the frame together with the $1 \sigma$ dispersion estimated from the interquartile ranges. The plot assumes the default $\mathrm{CO}$ corrections.

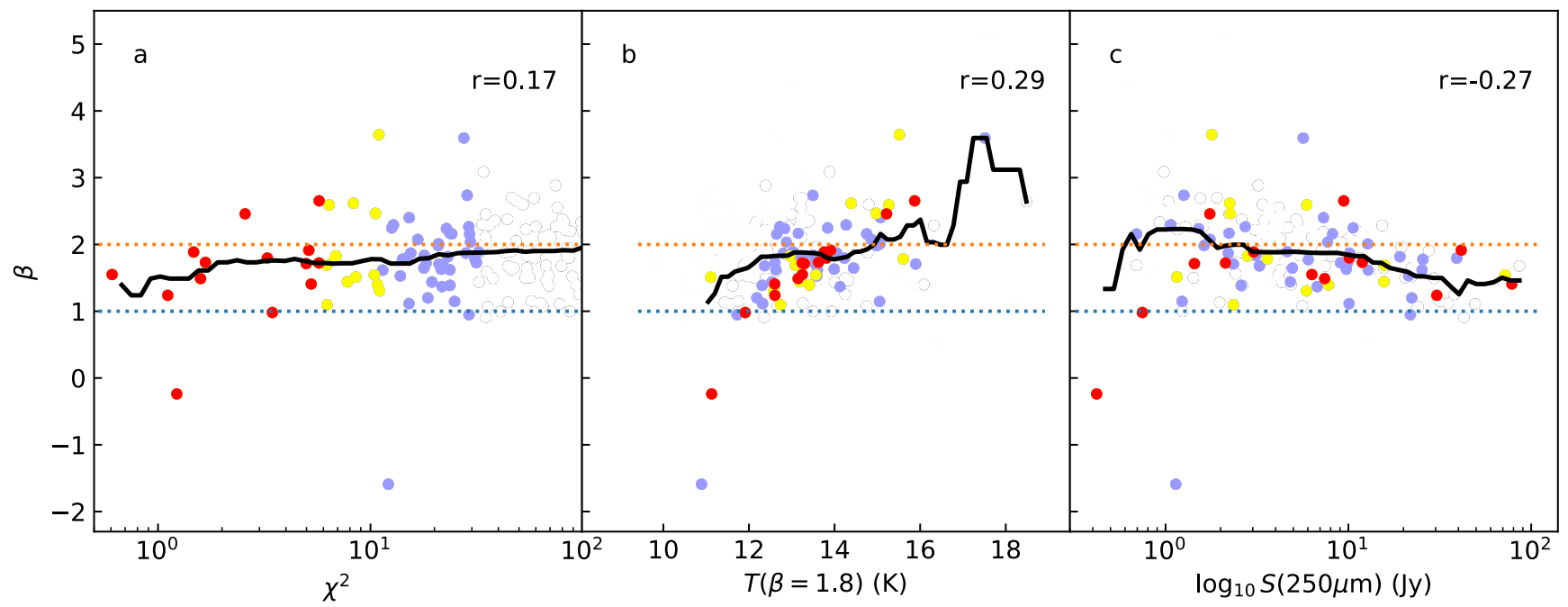

Fig. E.1. Dependence of $\beta$ estimates on $\chi^{2}$ values (frame $a$ ), SPIRE-derived colour temperatures for $\beta=1.8$ (frame $b$ ), and $250 \mu \mathrm{m}$ flux densities (frame $c$ ). The colours are the same as in Fig. 4. The linear correlation coefficients $r$ and median- $\beta$ curves are shown for the full set of plotted points.

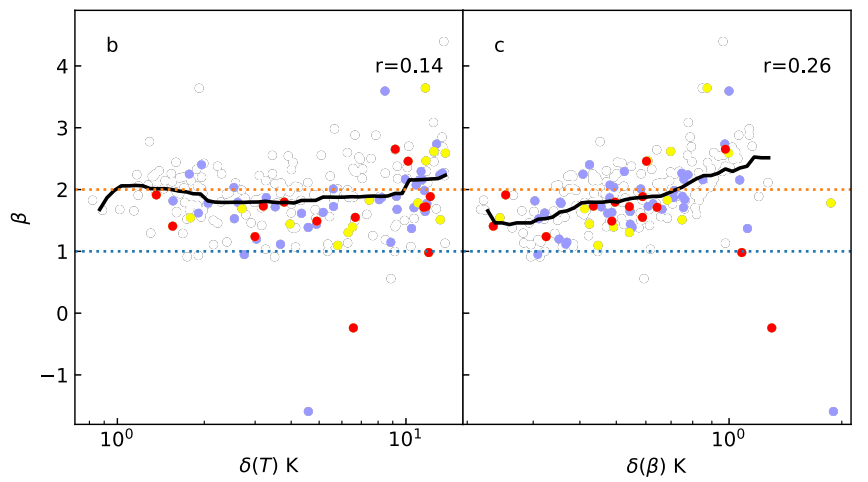

Fig. E.2. Estimates of $\beta$ as the function of the $T$ and $\beta$ MCMC error estimates. The symbol colours are as in Fig. E.1, and the black lines show the median values as a function of the $x$-axis variable. The linear correlation coefficients $r$ are given in the figure. 
A\&A 612, A71 (2018)
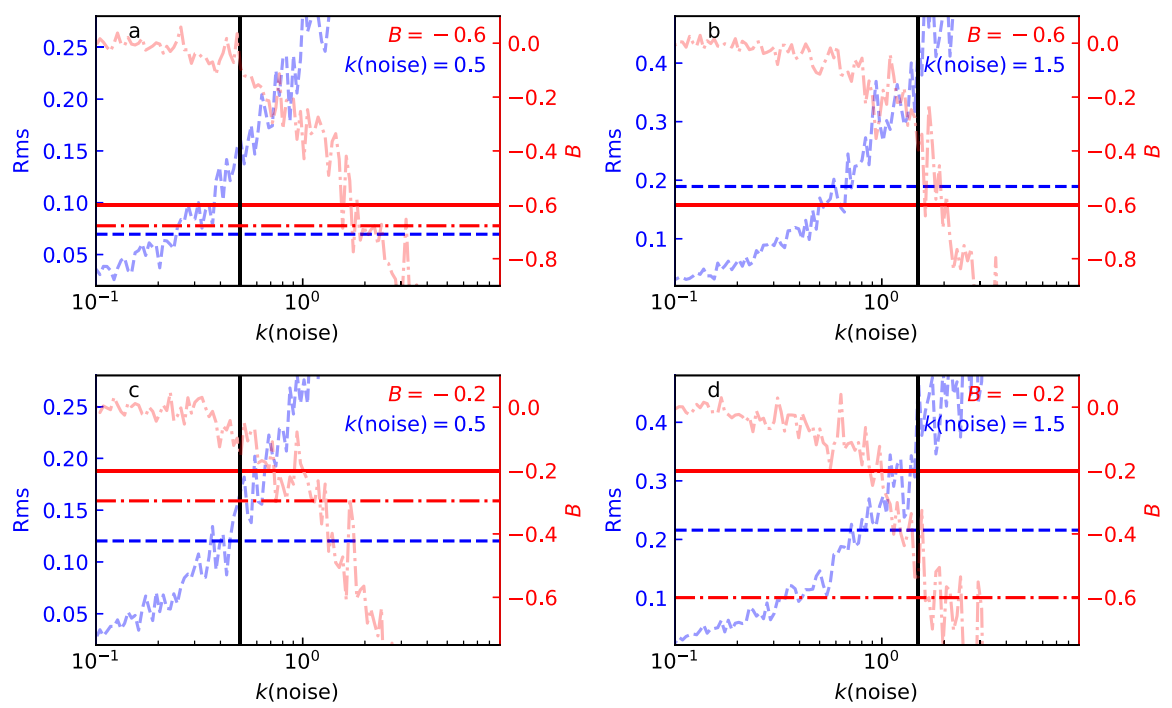

Fig. G.1. Analysis of a set of synthetic observations that follow an intrinsic $\beta(T) \propto T^{B}$ relation. The parameters $B$ and $k$ (noise) of the input simulation are given in the frames and are indicated by solid vertical and horizontal lines. The horizontal dot-dashed and dashed lines correspond to the values of $B$ and the $\beta \mathrm{rms}$ dispersion, respectively, which are estimated from one realisation of synthetic observations. The other curves show $B$ (dot-dashed red line) and the rms dispersion (blue dashed line) from simulations as a function of the noise scaling $k$ (noise).
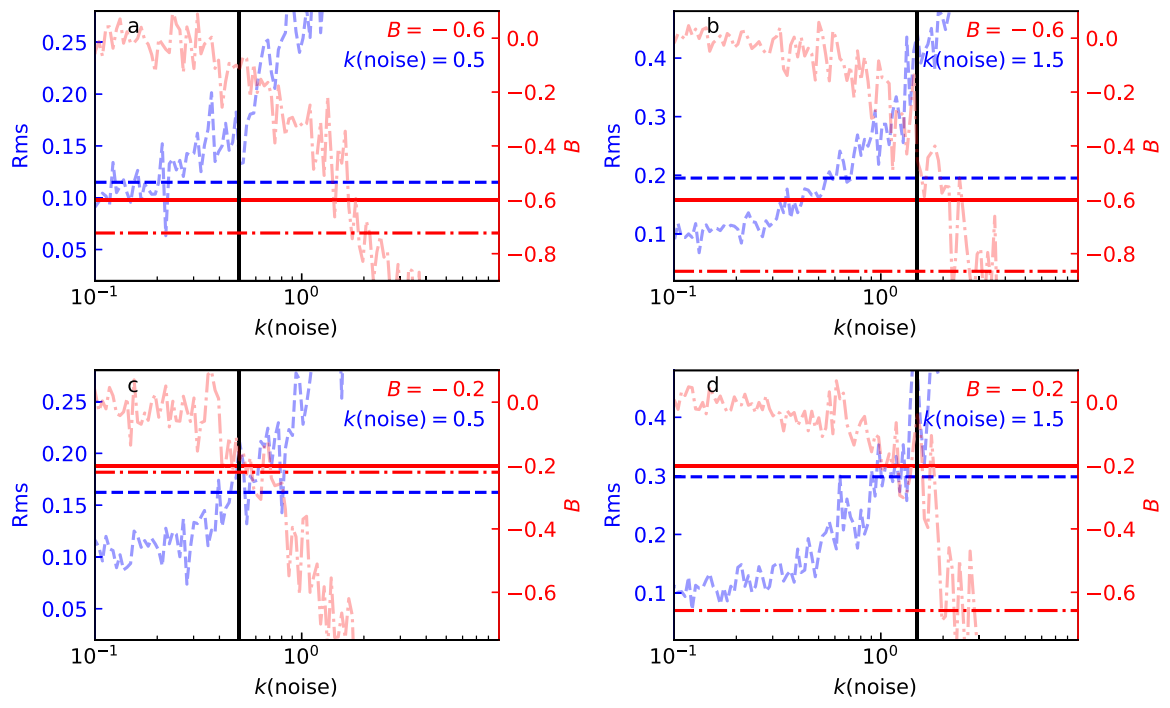

Fig. G.2. Same as Fig. G.1, but allowing for a dispersion $\sigma(\beta)=0.1$ of the intrinsic $\beta(T)$ relation. 
Table G.1. Statistics of spectral index $\beta$ distributions for different versions of the data.

\begin{tabular}{ccccccc}
\hline \hline$\theta_{\mathrm{F}}{ }^{a}\left({ }^{\prime \prime}\right)$ & Mask $^{b}$ & Aperture $^{c}$ & $\beta\left(3\right.$ bands, $\left.\mathrm{U}^{d}\right)$ & $\beta\left(3\right.$ bands, $\left.\mathrm{F}^{e}\right)$ & $\beta(4$ bands, F $)$ & Clumps $^{-1}$ \\
\hline $\mathrm{FCF}=2.41 \mathrm{Jy}(\mathrm{pW})^{-1}$ & arcsec $^{-2}$ & & & & \\
500 & $\mathrm{LM}$ & $\tau_{\text {all }}$ & $1.72,1.94,2.07$ & $1.69,1.99,2.29$ & $1.38,1.59,1.86$ & 27 \\
200 & $\mathrm{LM}$ & $\tau_{\text {all }}$ & $1.73,1.90,2.04$ & $1.75,2.09,2.50$ & $1.53,1.83,2.12$ & 29 \\
500 & $\mathrm{SM}$ & $\tau_{\text {all }}$ & $1.69,1.92,2.04$ & $1.73,1.91,2.22$ & $1.38,1.69,1.84$ & 17 \\
200 & $\mathrm{SM}$ & $\tau_{\text {all }}$ & $1.74,1.89,2.06$ & $1.83,1.99,2.41$ & $1.51,1.77,1.91$ & 18 \\
500 & $\mathrm{LM}$ & $\tau_{\text {high }}$ & $1.64,1.77,1.93$ & $1.70,1.88,2.17$ & $1.40,1.52,1.65$ & 22 \\
200 & $\mathrm{LM}$ & $\tau_{\text {high }}$ & $1.62,1.74,1.97$ & $1.88,2.03,2.29$ & $1.41,1.58,1.76$ & 23 \\
500 & $\mathrm{SM}$ & $\tau_{\text {high }}$ & $1.54,1.75,1.93$ & $1.55,1.72,1.92$ & $1.23,1.46,1.61$ & 14 \\
200 & $\mathrm{SM}$ & $\tau_{\text {high }}$ & $1.68,1.85,1.98$ & $1.92,2.07,2.22$ & $1.31,1.50,1.72$ & 15 \\
200 & $\mathrm{M} 850$ & $\tau_{\text {all }}$ & $1.68,1.82,2.00$ & $1.81,2.04,2.32$ & $1.42,1.67,1.86$ & 13 \\
200 & $\mathrm{No}$ & $\tau_{\text {all }}$ & $1.61,1.75,1.98$ & $2.12,2.69,3.08$ & $2.11,2.49,2.85$ & 16 \\
$\mathrm{FCF}=2.34 \mathrm{Jy}(\mathrm{pW})^{-1}$ & arcsec & & & & \\
500 & $\mathrm{LM}$ & $\tau_{\text {all }}$ & $1.69,1.90,2.06$ & $1.69,1.99,2.34$ & $1.43,1.63,1.91$ & 27 \\
200 & $\mathrm{LM}$ & $\tau_{\text {all }}$ & $1.73,1.90,2.04$ & $1.75,2.09,2.50$ & $1.58,1.84,2.12$ & 29 \\
500 & $\mathrm{SM}$ & $\tau_{\text {all }}$ & $1.63,1.92,2.04$ & $1.73,1.89,2.17$ & $1.48,1.72,1.88$ & 17 \\
200 & $\mathrm{SM}$ & $\tau_{\text {all }}$ & $1.69,1.89,2.06$ & $1.76,1.98,2.40$ & $1.53,1.78,1.96$ & 18 \\
500 & $\mathrm{LM}$ & $\tau_{\text {high }}$ & $1.64,1.79,1.93$ & $1.70,1.89,2.30$ & $1.44,1.56,1.66$ & 22 \\
200 & $\mathrm{LM}$ & $\tau_{\text {high }}$ & $1.62,1.75,1.99$ & $1.88,2.03,2.38$ & $1.48,1.62,1.78$ & 23 \\
500 & $\mathrm{SM}$ & $\tau_{\text {high }}$ & $1.53,1.73,1.89$ & $1.54,1.68,1.89$ & $1.28,1.46,1.64$ & 14 \\
200 & $\mathrm{SM}$ & $\tau_{\text {high }}$ & $1.68,1.85,1.98$ & $1.92,2.07,2.22$ & $1.35,1.55,1.75$ & 15 \\
200 & $\mathrm{M} 850$ & $\tau_{\text {all }}$ & $1.67,1.81,2.00$ & $1.79,2.04,2.37$ & $1.44,1.70,1.89$ & 13 \\
200 & $\mathrm{No}$ & $\tau_{\text {all }}$ & $1.61,1.74,1.97$ & $2.15,2.71,3.09$ & $2.09,2.52,3.00$ & 16 \\
\hline$\beta$
\end{tabular}

Notes. The listed $\beta$ values are the $25 \%, 50 \%$, and $75 \%$ percentiles for the clumps in the $P\left(\chi^{2}\right)=25 \%$ samples. ${ }^{(a)}$ Filtering scale in the SCUBA-2 map making. ${ }^{(b)}$ Small or large masks made based on Herschel data. See Appendix C. ${ }^{(c)}$ Apertures corresponding to Fellwalker clump footprints $\left(\tau_{\text {all }}\right)$ or, within them, only pixels above the median $\tau(250 \mu \mathrm{m})$ value $\left(\tau_{\text {high }}\right){ }^{(d)}$ U refers to original SPIRE data. ${ }^{(e)}$ F refers to SPIRE maps processed through the SCUBA-2 pipeline. 
Appendix H: Additional tables

Table H.1. SCUBA-2 fields.

\begin{tabular}{|c|c|c|c|c|c|c|c|c|}
\hline Field & $l$ & $b$ & $\begin{array}{c}\text { RA } \\
(\mathrm{J} 2000.0)\end{array}$ & $\begin{array}{c}\text { Dec } \\
(\mathrm{J} 2000.0)\end{array}$ & Obs.Id. & $\begin{array}{c}\text { Cover } \\
(\%)\end{array}$ & $\begin{array}{l}\mathrm{S}(250 \mu \mathrm{m}) \\
\left(\mathrm{MJy} \mathrm{sr}^{-1}\right)\end{array}$ & $\mathrm{CO}^{a}$ \\
\hline G003.7+18.3A1 & 3.74 & 18.34 & $16: 48: 48.2$ & $-15: 34: 40.1$ & 1342267754,1342267755 & 100 & 86.6 & \\
\hline G004.4+15.9A1 & 4.41 & 15.91 & $16: 58: 34.7$ & $-16: 28: 49.3$ & 1342267756,1342267757 & 46 & 27.9 & \\
\hline G007.5+21.1A1 & 7.54 & 21.08 & $16: 48: 13.6$ & $-11: 04: 32.1$ & 1342267724,1342267725 & 100 & 67.3 & \\
\hline $\mathrm{G} 007.8+21.1 \mathrm{~A} 1$ & 7.82 & 21.10 & $16: 48: 44.7$ & $-10: 51: 01.3$ & 1342267724,1342267725 & 100 & 61.9 & \\
\hline G038.3-00.9A1 & 38.35 & -0.95 & $19: 04: 44.7$ & $+04: 23: 03.4$ & 1342207026,1342207027 & 100 & 314.7 & \\
\hline G070.4-01.5A1 & 70.55 & -1.58 & $20: 15: 08.3$ & $+32: 02: 07.5$ & 1342219987 & 100 & 267.5 & \\
\hline G070.7-00.6A1 & 70.75 & -0.66 & $20: 12: 02.8$ & $+32: 42: 38.8$ & 1342219987 & 99 & 241.3 & \\
\hline G074.1+00.1A1 & 74.13 & 0.11 & 20:18:01.0 & $+35: 57: 18.8$ & 1342244190,1342244191 & 100 & 286.8 & \\
\hline G111.6+20.2A1 & 111.68 & 20.20 & $20: 57: 37.8$ & $+77: 35: 36.9$ & 1342198861,1342198862 & 100 & 64.1 & \\
\hline G113.4+16.9A1 & 113.43 & 17.01 & $21: 59: 43.3$ & $+76: 35: 35.4$ & 1342188679,1342188680 & 100 & 58.8 & \\
\hline G115.9+09.4A1 & 115.94 & 9.47 & $23: 24: 09.3$ & $+71: 08: 30.0$ & 1342222598 & 100 & 47.1 & $\mathrm{Z}$ \\
\hline $\mathrm{G} 127.67+2.65$ & 127.69 & 2.65 & $01: 36: 47.8$ & $+65: 06: 12.1$ & 1342203610 & 100 & 68.6 & \\
\hline G127.88+2.68 & 127.89 & 2.68 & $01: 38: 47.2$ & $+65: 05: 40.0$ & 1342203610 & 100 & 64.4 & $\mathrm{Z}$ \\
\hline $\mathrm{G} 130.1+11.0 \mathrm{~A} 1$ & 130.11 & 11.12 & $02: 28: 27.1$ & $+72: 37: 29.9$ & 1342216918 & 78 & 56.7 & \\
\hline $\mathrm{G} 130.1+11.0 \mathrm{~A} 2$ & 130.22 & 11.05 & $02: 29: 21.2$ & $+72: 31: 26.1$ & 1342216918 & 93 & 40.2 & \\
\hline G130.3+11.2A1 & 130.40 & 11.27 & $02: 32: 39.3$ & $+72: 39: 26.4$ & 1342216918 & 100 & 68.0 & \\
\hline G131.7+09.7A1 & 131.75 & 9.68 & 02:40:04.2 & $+70: 40: 26.8$ & 1342203609 & 100 & 43.5 & $\mathrm{Z}$ \\
\hline G132.0+08.8A1 & 132.10 & 8.77 & $02: 39: 25.3$ & $+69: 41: 36.2$ & 1342216919 & 100 & 50.6 & $\mathrm{Z}$ \\
\hline G132.0+08.9A1 & 132.05 & 8.97 & $02: 39: 49.3$ & $+69: 53: 40.0$ & 1342216919 & 100 & 65.3 & $\mathrm{Z}$ \\
\hline G144.6+00.1A1 & 144.71 & 0.22 & $03: 37: 14.4$ & $+55: 54: 57.1$ & 1342226995,1342226996 & 100 & 152.0 & $\bar{Z}$ \\
\hline G144.8+00.7A1 & 144.88 & 0.78 & $03: 40: 34.9$ & $+56: 15: 53.3$ & 1342226995,1342226996 & 100 & 103.7 & $\mathrm{Z}$ \\
\hline G148.2+00.4A1 & 148.19 & 0.39 & $03: 57: 01.7$ & $+53: 54: 06.9$ & 1342239047,1342239048 & 100 & 119.9 & $\mathrm{Z}$ \\
\hline $\mathrm{G} 149.2+03.0 \mathrm{~A} 2$ & 149.28 & 3.05 & $04: 14: 51.2$ & $+55: 09: 11.6$ & 1342216920 & 39 & 114.9 & $\mathrm{Z}$ \\
\hline $\mathrm{G} 149.41+3.38$ & 149.42 & 3.39 & $04: 17: 12.6$ & $+55: 17: 47.9$ & 1342216920 & 100 & 94.0 & $\mathrm{Z}$ \\
\hline $\mathrm{G} 149.60+3.45$ & 149.60 & 3.45 & $04: 18: 28.4$ & $+55: 12: 57.0$ & 1342216920 & 100 & 103.0 & $\mathrm{Z}$ \\
\hline G149.68+3.56 & 149.69 & 3.57 & 04:19:27.0 & $+55: 14: 29.1$ & 1342216920 & 100 & 99.2 & $\mathrm{Z}$ \\
\hline $\mathrm{G} 150.2+03.9 \mathrm{~B} 1$ & 150.29 & 3.92 & $04: 24: 11.1$ & $+55: 03: 34.2$ & 1342214702 & 100 & 105.3 & $\bar{Z}$ \\
\hline $\mathrm{G} 150.2+03.9 \mathrm{~B} 3$ & 150.21 & 3.96 & $04: 23: 56.4$ & $+55: 08: 45.8$ & 1342214702 & 100 & 88.2 & $\mathrm{Z}$ \\
\hline G150.4+03.9A1 & 150.44 & 4.04 & $04: 25: 29.1$ & $+55: 02: 11.9$ & 1342214702 & 100 & 91.7 & $\mathrm{Z}$ \\
\hline $\mathrm{G} 150.4+03.9 \mathrm{~A} 2$ & 150.36 & 3.97 & $04: 24: 45.1$ & $+55: 03: 06.4$ & 1342214702 & 100 & 100.7 & $\mathrm{Z}$ \\
\hline G151.4+03.9A1 & 151.45 & 3.96 & 04:29:56.1 & $+54: 15: 17.9$ & 1342203607 & 100 & 112.6 & $\mathrm{Z}$ \\
\hline $\mathrm{G} 154.0+05.0 \mathrm{~A} 1$ & 154.07 & 5.09 & $04: 47: 21.8$ & $+53: 03: 08.3$ & 1342216921 & 100 & 59.0 & $\mathrm{Z}$ \\
\hline G155.45-14.60 & 155.46 & -14.60 & $03: 35: 51.2$ & $+37: 40: 49.4$ & 1342226626 & 100 & 74.6 & \\
\hline G156.9-08.4A1 & 156.94 & -8.50 & $04: 01: 46.7$ & $+41: 28: 24.5$ & 1342203615 & 100 & 64.0 & M \\
\hline G157.12-8.72 & 157.13 & -8.71 & 04:01:46.9 & $+41: 11: 26.9$ & 1342203615 & 100 & 85.2 & M \\
\hline G157.93-2.51 & 157.93 & -2.50 & $04: 28: 35.8$ & $+45: 06: 46.8$ & 1342216923 & 100 & 58.8 & \\
\hline G158.8-21.6A1 & 158.85 & -21.67 & $03: 27: 40.2$ & $+30: 06: 00.4$ & 1342190326,1342190327 & 100 & 81.3 & \\
\hline G158.8-34.1A1 & 158.95 & -34.19 & $02: 55: 58.1$ & $+19: 49: 49.5$ & 1342239263,1342239264 & 100 & 62.9 & \\
\hline G159.0-08.4A1 & 159.03 & -8.46 & 04:09:59.0 & $+40: 06: 15.4$ & 1342239276,1342239277 & 100 & 80.9 & M \\
\hline G159.0-08.4A3 & 159.02 & -8.33 & $04: 10: 25.0$ & $+40: 12: 37.2$ & 1342239276,1342239277 & 100 & 69.1 & M \\
\hline G159.1-08.7A1 & 159.16 & -8.76 & $04: 09: 24.3$ & $+39: 47: 52.0$ & 1342239276,1342239277 & 100 & 55.3 & \\
\hline G159.21-34.28 & 159.22 & -34.27 & $02: 56: 33.9$ & $+19: 38: 18.7$ & 1342239263,1342239264 & 100 & 91.4 & \\
\hline G159.23-34.51 & 159.23 & -34.50 & $02: 56: 03.6$ & $+19: 26: 14.7$ & 1342239263,1342239264 & 100 & 98.9 & \\
\hline G159.4-34.3A1 & 159.39 & -34.39 & $02: 56: 47.0$ & $+19: 27: 44.7$ & 1342239263,1342239264 & 100 & 68.4 & \\
\hline G159.6-19.6A1 & 159.67 & -19.59 & $03: 36: 26.0$ & $+31: 15: 50.3$ & 1342214504,1342214505 & 100 & 117.5 & M \\
\hline G159.6-19.6A2 & 159.60 & -19.67 & $03: 35: 57.2$ & $+31: 14: 56.7$ & 1342214504,1342214505 & 100 & 93.9 & M \\
\hline G159.6-19.6A3 & 159.72 & -19.71 & $03: 36: 14.9$ & $+31: 08: 42.9$ & 1342214504,1342214505 & 100 & 104.8 & M \\
\hline G159.7-19.6A1 & 159.77 & -19.63 & $03: 36: 40.6$ & $+31: 10: 47.0$ & 1342214504,1342214505 & 100 & 118.9 & \\
\hline G160.6-16.7A1 & 160.62 & -16.72 & $03: 48: 19.7$ & $+32: 54: 57.5$ & 1342214504,1342214505 & 100 & 74.0 & M \\
\hline G160.8-09.4A1 & 160.83 & -9.42 & $04: 13: 12.6$ & $+38: 10: 54.4$ & 1342203616 & 100 & 76.5 & \\
\hline G160.8-09.4A2 & 160.81 & -9.49 & $04: 12: 52.9$ & $+38: 09: 04.1$ & 1342203616 & 100 & 74.3 & \\
\hline G161.3-09.3A1 & 161.34 & -9.32 & $04: 15: 22.3$ & $+37: 54: 36.1$ & 1342203616 & 100 & 77.0 & \\
\hline G161.56-9.29 & 161.57 & -9.28 & 04:16:19.1 & $+37: 46: 23.8$ & 1342203616 & 100 & 71.1 & \\
\hline
\end{tabular}

Notes. The columns are (1) name of the field, (2)-(5) centre coordinates, (6) Herschel observation ID, (7) fraction of SCUBA-2 map covered by Herschel observations, (8) median $250 \mu \mathrm{m}$ surface brightness, and (9) reference to CO observations. ${ }^{(a)} \mathrm{M}=\mathrm{Meng}$ et al. (2013), $\mathrm{Z}=\mathrm{Zhang}$ et al. (2016). 
Table H.1. continued.

\begin{tabular}{|c|c|c|c|c|c|c|c|c|}
\hline Field & $l$ & $b$ & $\begin{array}{c}\text { RA } \\
(\mathrm{J} 2000.0)\end{array}$ & $\begin{array}{c}\text { Dec } \\
(\mathrm{J} 2000.0)\end{array}$ & Obs.Id. & $\begin{array}{c}\text { Cover } \\
(\%)\end{array}$ & $\begin{array}{l}\mathrm{S}(250 \mu \mathrm{m}) \\
\left(\mathrm{MJy} \mathrm{sr}^{-1}\right)\end{array}$ & $\mathrm{CO}^{a}$ \\
\hline G162.4-08.7A1 & 162.45 & -8.70 & $04: 21: 30.3$ & $+37: 34: 12.0$ & 1342239278,1342239279 & 100 & 84.5 & M \\
\hline G163.32-8.41 & 163.32 & -8.40 & $04: 25: 37.4$ & $+37: 09: 28.0$ & 1342205047,1342205048 & 100 & 73.8 & M \\
\hline G163.68-8.33 & 163.68 & -8.31 & $04: 27: 10.3$ & $+36: 57: 29.4$ & 1342205047,1342205048 & 100 & 79.3 & \\
\hline G163.82-8.33 & 163.82 & -8.32 & $04: 27: 36.5$ & $+36: 50: 59.8$ & 1342205047,1342205048 & 100 & 79.7 & \\
\hline G164.1-08.8A1 & 164.15 & -8.88 & $04: 26: 41.8$ & $+36: 13: 41.1$ & 1342205047,1342205048 & 100 & 63.6 & M \\
\hline G164.11-8.17 & 164.12 & -8.15 & $04: 29: 12.2$ & $+36: 45: 06.7$ & 1342205047,1342205048 & 100 & 81.1 & \\
\hline G164.26-8.39 & 164.26 & -8.37 & $04: 28: 54.5$ & $+36: 29: 47.3$ & 1342205047,1342205048 & 100 & 87.7 & \\
\hline G165.1-07.5A1 & 165.18 & -7.53 & $04: 35: 02.3$ & $+36: 23: 41.8$ & 1342240279,1342240314 & 100 & 60.1 & \\
\hline G165.3-07.5A1 & 165.36 & -7.50 & $04: 35: 44.8$ & $+36: 17: 24.3$ & 1342240279,1342240314 & 100 & 56.1 & \\
\hline G165.6-09.1A1 & 165.71 & -9.12 & $04: 30: 59.7$ & $+34: 56: 20.8$ & 1342240279,1342240314 & 100 & 68.9 & \\
\hline G167.2-15.3A1 & 167.24 & -15.33 & $04: 14: 30.7$ & $+29: 35: 06.5$ & 1342190616,1342202090 & 100 & 33.9 & \\
\hline G168.1-16.3A1 & 168.15 & -16.40 & $04: 13: 50.0$ & $+28: 12: 25.8$ & 1342190616,1342202090 & 100 & 111.6 & M \\
\hline G169.1-01.1A1 & 169.16 & -1.15 & $05: 12: 20.1$ & $+37: 19: 43.6$ & 1342250233,1342266671 & 81 & 70.8 & $\mathrm{Z}$ \\
\hline G170.0-16.1A1 & 169.96 & -16.17 & $04: 19: 58.2$ & $+27: 06: 38.3$ & 1342190616,1342202090 & 100 & 66.6 & M \\
\hline G170.1-16.0A1 & 170.14 & -16.06 & $04: 20: 51.7$ & $+27: 03: 12.9$ & 1342190616,1342202090 & 100 & 61.9 & \\
\hline G170.8-18.3A1 & 170.86 & -18.36 & $04: 15: 27.9$ & $+24: 59: 13.5$ & 1342190617,1342190618 & 100 & 51.6 & \\
\hline G170.9-15.8A1 & 171.03 & -15.79 & $04: 24: 22.2$ & $+26: 36: 40.1$ & 1342190616,1342202090 & 100 & 58.6 & \\
\hline G171.14-17.57 & 171.15 & -17.57 & $04: 18: 51.3$ & $+25: 19: 33.0$ & 1342204860,1342204861 & 100 & 60.7 & \\
\hline G171.8-15.3A1 & 171.80 & -15.38 & $04: 27: 56.8$ & $+26: 19: 33.8$ & 1342239280,1342239281 & 100 & 53.2 & \\
\hline G172.8-14.7A1 & 172.91 & -14.74 & $04: 33: 12.6$ & $+25: 56: 30.2$ & 1342239280,1342239281 & 100 & 72.9 & M \\
\hline G173.1-13.3A1 & 173.14 & -13.32 & $04: 38: 41.7$ & $+26: 41: 30.4$ & 1342202252,1342202253 & 100 & 66.8 & \\
\hline G173.3-16.2A1 & 173.42 & -16.29 & $04: 29: 27.8$ & $+24: 33: 29.3$ & 1342190654,1342190655 & 100 & 119.6 & \\
\hline G173.9-13.7A1 & 173.96 & -13.75 & $04: 39: 29.4$ & $+25: 48: 17.9$ & 1342202252,1342202253 & 100 & 159.0 & \\
\hline G174.0-15.8A1 & 174.06 & -15.83 & $04: 32: 44.8$ & $+24: 23: 28.1$ & 1342190654,1342190655 & 100 & 94.8 & M \\
\hline G174.4-15.7A1 & 174.41 & -15.76 & $04: 33: 57.7$ & $+24: 10: 46.4$ & 1342190654,1342190655 & 100 & 63.9 & \\
\hline G174.7-15.4A1 & 174.73 & -15.50 & $04: 35: 42.2$ & $+24: 06: 39.4$ & 1342190652,1342190653 & 100 & 105.2 & \\
\hline G174.8-17.1A1 & 174.88 & -17.08 & $04: 30: 49.5$ & $+22: 58: 36.2$ & 1342190652,1342190653 & 100 & 46.2 & \\
\hline $\mathrm{G} 175.2+01.2 \mathrm{~A} 2$ & 175.17 & 1.29 & $05: 38: 48.5$ & $+33: 43: 18.2$ & 1342250332,1342250333 & 100 & 38.8 & $\mathrm{Z}$ \\
\hline G175.4-16.8A2 & 175.51 & -16.78 & 04:33:30.1 & $+22: 43: 05.0$ & 1342190652,1342190653 & 100 & 85.2 & \\
\hline G177.6-20.3A1 & 177.62 & -20.35 & $04: 27: 16.9$ & $+18: 53: 32.9$ & 1342202250,1342202251 & 100 & 79.3 & \\
\hline G181.8+00.3A1 & 181.92 & 0.35 & $05: 51: 28.1$ & $+27: 28: 02.5$ & 1342250798,1342250799 & 100 & 82.7 & \\
\hline G201.2+00.4A1 & 201.27 & 0.44 & $06: 31: 32.0$ & $+10: 33: 54.8$ & 1342204419,1342204420 & 100 & 123.7 & \\
\hline $\mathrm{G} 201.83+2.83$ & 201.83 & 2.83 & $06: 41: 13.5$ & $+11: 10: 09.6$ & 1342228342 & 100 & 48.6 & \\
\hline $\mathrm{G} 202.00+2.65$ & 202.00 & 2.65 & $06: 40: 52.1$ & $+10: 56: 00.0$ & 1342228342 & 100 & 69.2 & \\
\hline G202.54+2.46 & 202.53 & 2.46 & $06: 41: 10.9$ & $+10: 22: 34.6$ & 1342228342 & 100 & 131.3 & \\
\hline G204.4-11.3A2 & 204.50 & -11.36 & $05: 55: 37.5$ & $+02: 11: 15.3$ & 1342205074,1342205075 & 100 & 100.5 & \\
\hline G204.8-13.8A1 & 204.85 & -13.89 & $05: 47: 22.9$ & $+00: 41: 23.2$ & 1342215982,1342215983 & 100 & 148.5 & \\
\hline G210.90-36.55 & 210.89 & -36.55 & $04: 35: 09.3$ & $-14: 14: 41.4$ & 1342216940 & 100 & 57.2 & \\
\hline G215.44-16.38 & 215.43 & -16.38 & $05: 57: 02.5$ & $-09: 32: 28.3$ & 1342203631 & 100 & 43.0 & \\
\hline $\mathrm{G} 216.76-2.58$ & 216.75 & -2.57 & $06: 49: 13.7$ & $-04: 34: 27.1$ & 1342219956 & 100 & 71.4 & \\
\hline G219.13-9.72 & 219.12 & -9.72 & $06: 27: 43.0$ & $-09: 52: 51.8$ & 1342227708 & 100 & 58.4 & \\
\hline G219.22-10.02 & 219.22 & -10.02 & $06: 26: 47.7$ & $-10: 05: 36.5$ & 1342227708 & 100 & 59.8 & \\
\hline G219.36-9.71 & 219.36 & -9.71 & $06: 28: 10.3$ & $-10: 04: 58.6$ & 1342227708 & 100 & 84.3 & \\
\hline
\end{tabular}


Table H.2. Distance estimates.

\begin{tabular}{|c|c|c|c|c|}
\hline SCUBA-2 field & $\begin{array}{l}d\left(\mathrm{PGCC}^{a}\right. \\
(\mathrm{pc})\end{array}$ & GCC field $^{b}$ & $\begin{array}{l}d(\mathrm{GCC})^{c} \\
(\mathrm{pc})\end{array}$ & $\begin{array}{l}\text { Associated clouds } \\
(\mathrm{pc})\end{array}$ \\
\hline G003.7+18.3A1 & 120 & - & - & \\
\hline G004.4+15.9A1 & - & - & - & \\
\hline G007.5+21.1A1 & 120 & - & - & LDN234: 160, MBM 148 \\
\hline G007.8+21.1A1 & 120 & - & - & LDN234: 160 \\
\hline G038.3-00.9A1 & 1200 & - & - & \\
\hline G070.4-01.5A1 & 2090 & G70.10-1.69 & 2090 & \\
\hline G070.7-00.6A1 & 2260 & - & - & LDN841 \\
\hline G074.1+00.1A1 & 3450 & - & - & \\
\hline G111.6+20.2A1 & - & - & - & LDN1228: 170, MBM 162 \\
\hline G113.4+16.9A1 & - & - & - & LDN1241 \\
\hline G115.9+09.4A1 & 1000 & G115.93+9.47 & 1000 & \\
\hline $\mathrm{G} 127.67+2.65$ & 800 & $\mathrm{G} 127.79+2.66$ & 800 & CB11 \\
\hline G127.88+2.68 & 800 & G127.79+2.66 & 800 & CB12 \\
\hline G130.1+11.0A1 & 600 & $\mathrm{G} 130.37+11.26$ & 600 & LDN1340A \\
\hline G130.1+11.0A2 & 600 & G130.37+11.26 & 600 & LDN1340A \\
\hline $\mathrm{G} 130.3+11.2 \mathrm{~A} 1$ & 600 & G130.37+11.26 & 600 & LDN1340C \\
\hline G131.7+09.7A1 & 1070 & G131.65+9.75 & 1070 & \\
\hline G132.0+08.8A1 & 850 & G132.12+8.95 & 850 & \\
\hline G132.0+08.9A1 & 850 & G132.12+8.95 & 850 & \\
\hline G144.6+00.1A1 & - & - & - & \\
\hline G144.8+00.7A1 & - & - & - & \\
\hline G148.2+00.4A1 & - & - & - & \\
\hline $\mathrm{G} 149.2+03.0 \mathrm{~A} 2$ & - & G149.67+3.56 & 170 & \\
\hline G149.41+3.38 & 170 & G149.67+3.56 & 170 & LDN1394, B8 \\
\hline G149.60+3.45 & 170 & G149.67+3.56 & 170 & LDN1400H, MLB71, B9 \\
\hline G149.68+3.56 & 170 & G149.67+3.56 & 170 & \\
\hline G150.2+03.9B1 & 170 & G150.47+3.93 & 170 & LDN1399: 170, LDN1400 \\
\hline $\mathrm{G} 150.2+03.9 \mathrm{~B} 3$ & 170 & $\mathrm{G} 150.47+3.93$ & 170 & LDN1399: 170 \\
\hline G150.4+03.9A1 & 170 & G150.47+3.93 & 170 & LDN1400 \\
\hline G150.4+03.9A2 & 170 & G150.47+3.93 & 170 & $\begin{array}{c}\text { LDN1399: 170, } \\
\text { LDN1400, MLB72, MLB73, MLB74 }\end{array}$ \\
\hline G151.4+03.9A1 & 170 & G151.45+3.95 & 170 & B12, LDN1407, MLB77: 170, LDN1400 \\
\hline G154.0+05.0A1 & 170 & G154.08+5.23 & 170 & LDN1426: 170, LDN 1426 \\
\hline G155.45-14.60 & 350 & G155.80-14.24 & 350 & LDN1434: 350 \\
\hline G156.9-08.4A1 & 150 & G157.08-8.68 & 150 & \\
\hline G157.12-8.72 & 150 & G157.08-8.68 & 150 & LDN1443 \\
\hline G157.93-2.51 & 2500 & G157.92-2.28 & 2500 & \\
\hline G158.8-21.6A1 & 140 & - & - & LDN1452: 280, LDN1455 \\
\hline G158.8-34.1A1 & 325 & G159.23-34.51 & 325 & LDN1454: 200, MBM12: 70 \\
\hline G159.0-08.4A1 & 140 & - & - & LDN1459: 350 \\
\hline G159.0-08.4A3 & 140 & - & - & LDN1459: 350 \\
\hline G159.1-08.7A1 & 140 & - & - & LDN1459: 350 \\
\hline G159.21-34.28 & 325 & G159.23-34.51 & 325 & LDN1457: 90, MBM12: 70 \\
\hline G159.23-34.51 & 325 & G159.23-34.51 & 325 & LDN1457: 90, MBM12: 70 \\
\hline G159.4-34.3A1 & 325 & G159.23-34.51 & 325 & LDN1457: 90, MBM12: 70 \\
\hline G159.6-19.6A1 & 140 & - & - & \\
\hline G159.6-19.6A2 & 140 & - & - & \\
\hline G159.6-19.6A3 & 140 & - & - & \\
\hline G159.7-19.6A1 & 140 & - & - & \\
\hline G160.6-16.7A1 & 140 & - & - & LDN1471: 350, B5 \\
\hline G160.8-09.4A1 & 140 & - & - & \\
\hline G160.8-09.4A2 & 140 & - & - & \\
\hline G161.3-09.3A1 & 250 & G161.55-9.30 & 250 & \\
\hline G161.56-9.29 & 250 & G161.55-9.30 & 250 & \\
\hline G162.4-08.7A1 & 140 & - & - & LDN1478: 350 \\
\hline
\end{tabular}

Notes. ${ }^{(a)}$ Distance estimate in the PGCC catalogue. ${ }^{(b)}$ Overlapping GCC Herschel field. ${ }^{(c)}$ Distance estimate in Montillaud et al. (2015). ${ }^{(d)}$ Known clouds within half a degree distance of the field centre. The references are Barnard et al. (1927) (B), Clemens \& Barvainis (1988) (CB), Lynds (1962) (LDN), Magnani et al. (1985) (MBM), and Myers et al. (1983) (MLB). Possible literature distance estimates given after a colon. 
Table H.2. continued.

\begin{tabular}{|c|c|c|c|c|}
\hline SCUBA-2 field & $\begin{array}{c}d\left(\mathrm{PGCC}^{a}\right. \\
(\mathrm{pc})\end{array}$ & GCC field ${ }^{b}$ & $\begin{array}{l}d(\mathrm{GCC})^{c} \\
(\mathrm{pc})\end{array}$ & $\begin{array}{l}\text { Associated clouds }{ }^{d} \\
(\mathrm{pc})\end{array}$ \\
\hline G163.32-8.41 & 420 & - & - & LDN1478: 350 \\
\hline G163.68-8.33 & 420 & G163.82-8.44 & 420 & LDN1478: 350 \\
\hline G163.82-8.33 & 420 & G163.82-8.44 & 420 & \\
\hline G164.1-08.8A1 & 420 & - & - & \\
\hline G164.11-8.17 & 420 & G163.82-8.44 & 420 & \\
\hline G164.26-8.39 & 420 & G163.82-8.44 & 420 & \\
\hline G165.1-07.5A1 & 140 & - & - & LDN1483 \\
\hline G165.3-07.5A1 & 140 & - & - & LDN1483 \\
\hline G165.6-09.1A1 & 140 & - & - & \\
\hline G167.2-15.3A1 & 140 & - & - & \\
\hline G168.1-16.3A1 & 140 & - & - & LDN1495: 140 \\
\hline G169.1-01.1A1 & - & - & - & \\
\hline G170.0-16.1A1 & 140 & - & - & \\
\hline G170.1-16.0A1 & 230 & - & - & B213, LDN1521 \\
\hline G170.8-18.3A1 & 230 & - & - & B210, LDN1501: 140 \\
\hline G170.9-15.8A1 & 230 & - & - & B216, MLB14: 140 \\
\hline G171.14-17.57 & 230 & - & - & LDN1506 \\
\hline G171.8-15.3A1 & 230 & - & - & B217, LDN1521 \\
\hline G172.8-14.7A1 & 230 & - & - & B19, LDN1521: 140 \\
\hline G173.1-13.3A1 & 230 & - & - & \\
\hline G173.3-16.2A1 & 230 & - & - & LDN1524: 140 \\
\hline G173.9-13.7A1 & 230 & - & - & $\begin{array}{l}\text { B22, LDN1528:140, LDN1532, MLB26, } \\
\text { TMC1-A: 140, LDN1534: } 140\end{array}$ \\
\hline G174.0-15.8A1 & 230 & - & - & B18, LDN1529: 140, TMC-2, MLB23: 140 \\
\hline G174.4-15.7A1 & 230 & - & - & B18-3: 140 \\
\hline G174.7-15.4A1 & 230 & - & - & LDN1535: 140 \\
\hline G174.8-17.1A1 & 230 & - & - & \\
\hline $\mathrm{G} 175.2+01.2 \mathrm{~A} 2$ & 4590 & - & - & \\
\hline G175.4-16.8A2 & 230 & - & - & LDN1536, MLB24: 140 \\
\hline G177.6-20.3A1 & 230 & - & - & LDN1543, MBM107: 140 \\
\hline G181.8+00.3A1 & - & - & - & \\
\hline $\mathrm{G} 201.2+00.4 \mathrm{~A} 1$ & - & - & - & B37 \\
\hline $\mathrm{G} 201.83+2.83$ & 760 & $\mathrm{G} 202.02+2.85$ & 760 & \\
\hline $\mathrm{G} 202.00+2.65$ & 760 & $\mathrm{G} 202.02+2.85$ & 760 & \\
\hline $\mathrm{G} 202.54+2.46$ & 760 & $\mathrm{G} 202.02+2.85$ & 760 & \\
\hline G204.4-11.3A2 & 415 & - & - & \\
\hline G204.8-13.8A1 & 415 & - & - & NGC2071-North \\
\hline G210.90-36.55 & 140 & G210.90-36.55 & 140 & MBM20: 110, LDN1642: 100, 140 \\
\hline G215.44-16.38 & 415 & G215.44-16.38 & 1450 & \\
\hline G216.76-2.58 & 2400 & G216.76-2.58 & 2400 & \\
\hline G219.13-9.72 & 905 & G219.36-9.71 & 905 & LDN1652: 830 \\
\hline G219.22-10.02 & 905 & G219.36-9.71 & 905 & LDN1652: 830 \\
\hline G219.36-9.71 & 905 & G219.36-9.71 & 905 & LDN1652: 830 \\
\hline
\end{tabular}


Table H.3. Properties of PGCC clumps in the SCUBA-2 fields according to the PGCC catalogue.

\begin{tabular}{|c|c|c|c|c|c|c|c|c|c|}
\hline Field & $\mathrm{No}^{a}$ & $\begin{array}{c}\text { RA } \\
(\mathrm{J} 2000.0) \\
\end{array}$ & $\begin{array}{c}\text { Dec } \\
(\mathrm{J} 2000.0) \\
\end{array}$ & $\begin{array}{c}l \\
(\mathrm{deg}) \\
\end{array}$ & $\begin{array}{c}b \\
(\mathrm{deg}) \\
\end{array}$ & $\begin{array}{c}T(\beta=2) \\
(\mathrm{K})\end{array}$ & $\begin{array}{c}T \\
(\mathrm{~K}) \\
\end{array}$ & $\beta$ & $\begin{array}{c}N\left(\mathrm{H}_{2}\right) \\
\left(10^{21} \mathrm{~cm}^{-2}\right)\end{array}$ \\
\hline G003.7+18.3A1 & 1 & $16: 48: 53.8$ & $-15: 35: 34$ & 3.74 & 18.32 & $12.0(0.2)$ & $10.6(0.7)$ & $2.4(0.2)$ & $10.0(2.5)$ \\
\hline G004.4+15.9A1 & 1 & $16: 58: 36.1$ & $-16: 28: 56$ & 4.41 & 15.90 & $14.1(0.3)$ & $14.1(0.7)$ & $2.0(0.1)$ & - \\
\hline $\mathrm{G} 007.5+21.1 \mathrm{~A} 1$ & 1 & $16: 48: 08.2$ & $-11: 01: 20$ & 7.57 & 21.13 & $12.9(0.3)$ & $12.2(0.8)$ & $2.2(0.2)$ & $4.2(1.0)$ \\
\hline G007.8+21.1A1 & 1 & $16: 48: 46.6$ & $-10: 50: 58$ & 7.82 & 21.10 & $12.8(0.6)$ & $11.9(1.8)$ & $2.2(0.4)$ & $1.9(1.0)$ \\
\hline G038.3-00.9A1 & 1 & $19: 04: 40.8$ & $+04: 23: 45$ & 38.35 & -0.93 & $12.2(1.0)$ & - & - & $7.9(2.3)$ \\
\hline G070.4-01.5A1 & 1 & $20: 14: 47.2$ & $+31: 56: 56$ & 70.43 & -1.57 & $13.1(1.0)$ & - & - & $7.4(2.0)$ \\
\hline G070.7-00.6A1 & 1 & $20: 11: 52.6$ & $+32: 41: 56$ & 70.72 & -0.64 & $12.1(0.6)$ & $10.7(1.5)$ & $2.5(0.6)$ & $13.7(7.1)$ \\
\hline $\mathrm{G} 074.1+00.1 \mathrm{~A} 1$ & 1 & $20: 17: 51.1$ & $+35: 57: 37$ & 74.12 & 0.15 & $12.4(1.8)$ & - & - & $6.4(3.2)$ \\
\hline G111.6+20.2A1 & 1 & $20: 57: 18.5$ & $+77: 34: 05$ & 111.65 & 20.20 & $11.6(0.3)$ & $11.7(0.9)$ & $2.0(0.2)$ & $5.1(1.5)$ \\
\hline G113.4+16.9A1 & 1 & $21: 59: 47.2$ & $+76: 34: 00$ & 113.41 & 16.98 & $10.7(0.3)$ & $9.2(1.0)$ & $2.8(0.4)$ & $7.7(3.6)$ \\
\hline G115.9+09.4A1 & 1 & $23: 24: 04.0$ & $+71: 08: 41$ & 115.93 & 9.47 & $13.4(0.3)$ & $12.7(0.8)$ & $2.2(0.2)$ & $1.4(0.3)$ \\
\hline $\mathrm{G} 127.67+2.65$ & 1 & $01: 36: 40.5$ & $+65: 06: 04$ & 127.67 & 2.65 & $12.5(1.3)$ & $13.7(3.2)$ & $1.6(0.9)$ & - \\
\hline G127.88+2.68 & 1 & $01: 38: 40.6$ & $+65: 05: 32$ & 127.88 & 2.68 & $11.5(0.4)$ & $11.5(0.8)$ & $2.0(0.2)$ & $3.2(0.8)$ \\
\hline \multirow{2}{*}{ G130.1+11.0A1 } & 1 & $02: 28: 53.8$ & $+72: 33: 05$ & 130.17 & 11.06 & $12.7(0.4)$ & $13.3(0.8)$ & $1.9(0.2)$ & $1.8(0.4)$ \\
\hline & 2 & $02: 29: 16.1$ & $+72: 43: 09$ & 130.13 & 11.23 & $12.4(3.9)$ & - & - & $1.1(0.9)$ \\
\hline G130.1+11.0A2 & 1 & $02: 28: 53.8$ & $+72: 33: 05$ & 130.17 & 11.06 & $12.7(0.4)$ & $13.3(0.8)$ & $1.9(0.2)$ & $1.8(0.4)$ \\
\hline G130.3+11.2A1 & 1 & $02: 32: 22.6$ & $+72: 39: 27$ & 130.38 & 11.26 & $11.1(0.4)$ & $10.2(1.9)$ & $2.4(0.6)$ & $6.3(4.4)$ \\
\hline G131.7+09.7A1 & 1 & 02:40:04.8 & $+70: 41: 05$ & 131.74 & 9.69 & $12.1(0.3)$ & $11.7(0.7)$ & $2.1(0.2)$ & $3.1(0.7)$ \\
\hline $\mathrm{G} 132.0+08.8 \mathrm{~A} 1$ & 1 & 02:39:13.4 & $+69: 42: 01$ & 132.09 & 8.77 & $12.1(0.5)$ & $11.8(1.3)$ & $2.1(0.4)$ & $2.0(0.8)$ \\
\hline \multirow{2}{*}{$\mathrm{G} 132.0+08.9 \mathrm{~A} 1$} & 1 & $02: 39: 43.5$ & $+69: 54: 11$ & 132.04 & 8.97 & $12.5(1.3)$ & - & - & $1.6(0.6)$ \\
\hline & 2 & $02: 40: 29.1$ & $+69: 51: 12$ & 132.12 & 8.95 & - & - & - & - \\
\hline G144.6+00.1A1 & 1 & 03:37:00.4 & $+55: 54: 30$ & 144.69 & 0.19 & $11.7(1.3)$ & - & - & $2.2(0.9)$ \\
\hline G144.8+00.7A1 & 1 & $03: 40: 36.0$ & $+56: 16: 09$ & 144.88 & 0.78 & $11.8(1.7)$ & - & - & $2.1(1.1)$ \\
\hline $\mathrm{G} 148.2+00.4 \mathrm{~A} 1$ & 1 & $03: 57: 21.5$ & $+53: 52: 40$ & 148.24 & 0.40 & $11.6(1.9)$ & - & - & $2.3(1.3)$ \\
\hline $\mathrm{G} 149.2+03.0 \mathrm{~A} 2$ & 1 & $04: 14: 52.3$ & $+55: 11: 46$ & 149.25 & 3.08 & $13.4(2.4)$ & - & - & $1.9(1.1)$ \\
\hline G149.41+3.38 & 1 & 04:17:08.6 & $+55: 17: 46$ & 149.41 & 3.38 & $13.1(1.3)$ & - & - & $1.5(0.5)$ \\
\hline G149.60+3.45 & 1 & $04: 18: 24.7$ & $+55: 12: 55$ & 149.60 & 3.45 & $12.2(0.5)$ & $11.1(1.1)$ & $2.3(0.3)$ & $3.3(1.3)$ \\
\hline $\mathrm{G} 149.68+3.56$ & 1 & $04: 19: 23.0$ & $+55: 14: 27$ & 149.68 & 3.56 & $13.0(1.1)$ & - & - & $1.8(0.6)$ \\
\hline \multirow{2}{*}{$\mathrm{G} 150.2+03.9 \mathrm{~B} 1$} & 1 & & $+55: 07: 22$ & & 3.95 & $11.0(0.5)$ & $10.1(2.5)$ & $2.4(0.8)$ & $6.8(5.8)$ \\
\hline & 2 & $04: 23: 56.0$ & $+54: 57: 45$ & 150.34 & 3.83 & - & - & - & - \\
\hline G150.2+03.9B3 & 1 & 04:24:00.6 & $+55: 07: 22$ & 150.23 & 3.95 & $11.0(0.5)$ & $10.1(2.5)$ & $2.4(0.8)$ & $6.8(5.8)$ \\
\hline G150.4+03.9A 1 & 1 & $04: 25: 06.8$ & $+54: 56: 28$ & 150.47 & 3.93 & $12.5(1.6)$ & - & - & $4.9(2.2)$ \\
\hline G151.4+03.9A1 & 1 & $04: 29: 51.8$ & $+54: 15: 16$ & 151.45 & 3.95 & $11.7(0.3)$ & $10.1(0.9)$ & $2.6(0.4)$ & $9.2(3.5)$ \\
\hline G154.0+05.0A1 & 1 & $04: 47: 14.5$ & $+53: 02: 11$ & 154.07 & 5.07 & $11.4(0.3)$ & $10.2(0.9)$ & $2.4(0.3)$ & $4.1(1.7)$ \\
\hline G155.45-14.60 & 1 & $03: 35: 48.5$ & $+37: 40: 45$ & 155.45 & -14.60 & $11.5(0.4)$ & $9.8(1.3)$ & $2.7(0.6)$ & $7.3(4.0)$ \\
\hline G156.9-08.4A1 & 1 & 04:01:36.3 & $+41: 28: 31$ & 156.91 & -8.52 & $12.6(1.0)$ & - & - & $2.7(0.8)$ \\
\hline G157.12-8.72 & 1 & 04:01:42.6 & $+41: 11: 11$ & 157.12 & -8.72 & $13.0(2.3)$ & - & - & $2.9(1.7)$ \\
\hline G157.93-2.51 & 1 & $04: 28: 30.7$ & $+45: 06: 11$ & 157.93 & -2.51 & $12.6(3.3)$ & - & - & - \\
\hline \multirow[t]{2}{*}{ G158.8-21.6A1 } & 1 & 03:28:00.0 & $+30: 07: 55$ & 158.89 & -21.60 & $10.4(1.8)$ & - & - & $5.1(3.5)$ \\
\hline & 2 & 03:27:19.4 & $+30: 00: 40$ & 158.84 & -21.78 & - & - & - & - \\
\hline G158.8-34.1A1 & 1 & $02: 55: 43.0$ & $+19: 52: 32$ & 158.86 & -34.19 & $12.5(0.7)$ & $10.8(1.8)$ & $2.6(0.7)$ & $3.1(1.9)$ \\
\hline \multirow[t]{2}{*}{ G159.0-08.4A1 } & 1 & 04:09:47.6 & $+40: 07: 33$ & 158.99 & -8.47 & $13.4(1.4)$ & - & - & $2.0(0.7)$ \\
\hline & 2 & $04: 10: 19.5$ & $+40: 11: 51$ & 159.02 & -8.35 & - & - & - & - \\
\hline G159.0-08.4A3 & 1 & $04: 10: 19.5$ & $+40: 11: 51$ & 159.02 & -8.35 & - & - & - & - \\
\hline G159.1-08.7A1 & 1 & 04:09:21.3 & $+39: 48: 14$ & 159.15 & -8.76 & $12.4(0.7)$ & $12.0(1.7)$ & $2.1(0.5)$ & $1.7(0.8)$ \\
\hline G159.21-34.28 & 1 & $02: 56: 32.6$ & $+19: 38: 13$ & 159.21 & -34.28 & $12.9(0.5)$ & $12.0(1.6)$ & $2.3(0.6)$ & $3.0(1.5)$ \\
\hline G159.23-34.51 & 1 & 02:56:02.3 & $+19: 26: 13$ & 159.23 & -34.51 & $12.1(0.4)$ & $10.6(1.1)$ & $2.5(0.4)$ & $8.7(3.7)$ \\
\hline G159.4-34.3A1 & 1 & $02: 56: 53.7$ & $+19: 27: 53$ & 159.41 & -34.37 & $12.3(0.5)$ & $10.9(1.3)$ & $2.5(0.5)$ & $4.5(2.1)$ \\
\hline G159.6-19.6A1 & 1 & $03: 36: 37.8$ & $+31: 10: 58$ & 159.76 & -19.63 & - & - & - & - \\
\hline G159.6-19.6A2 & 1 & 03:35:53.7 & $+31: 12: 06$ & 159.62 & -19.71 & $12.7(1.9)$ & - & - & $3.2(1.6)$ \\
\hline \multirow[t]{2}{*}{ G159.6-19.6A3 } & 1 & $03: 35: 53.7$ & $+31: 12: 06$ & 159.62 & -19.71 & $12.7(1.9)$ & - & - & $3.2(1.6)$ \\
\hline & 2 & $03: 36: 37.8$ & $+31: 10: 58$ & 159.76 & -19.63 & - & - & - & - \\
\hline G159.7-19.6A1 & 1 & $03: 36: 37.8$ & $+31: 10: 58$ & 159.76 & -19.63 & - & - & - & - \\
\hline G160.6-16.7A1 & 1 & $03: 48: 23.5$ & $+32: 53: 39$ & 160.64 & -16.72 & - & - & - & - \\
\hline G160.8-09.4A1 & 1 & 04:13:05.7 & $+38: 08: 28$ & 160.85 & -9.46 & $12.6(4.0)$ & - & - & $1.0(0.9)$ \\
\hline G160.8-09.4A2 & 1 & 04:13:05.7 & $+38: 08: 28$ & 160.85 & -9.46 & $12.6(4.0)$ & - & - & $1.0(0.9)$ \\
\hline
\end{tabular}

Notes. ${ }^{(a)}$ Index within the field, used in Fig. C.1. 
Table H.3. continued.

\begin{tabular}{|c|c|c|c|c|c|c|c|c|c|}
\hline Field & $\mathrm{No}^{a}$ & $\begin{array}{c}\text { RA } \\
(\mathrm{J} 2000.0)\end{array}$ & $\begin{array}{c}\text { Dec } \\
(\mathrm{J} 2000.0)\end{array}$ & $\begin{array}{c}l \\
(\operatorname{deg})\end{array}$ & $\begin{array}{c}b \\
(\mathrm{deg})\end{array}$ & $\begin{array}{c}T(\beta=2) \\
(\mathrm{K})\end{array}$ & $\begin{array}{c}T \\
(\mathrm{~K}) \\
\end{array}$ & $\beta$ & $\begin{array}{c}N\left(\mathrm{H}_{2}\right) \\
\left(10^{21} \mathrm{~cm}^{-2}\right)\end{array}$ \\
\hline G161.3-09.3A1 & 1 & $04: 15: 23.9$ & $+37: 53: 03$ & 161.36 & -9.33 & $12.5(1.2)$ & $12.8(2.4)$ & $1.9(0.7)$ & $1.0(0.6)$ \\
\hline \multirow[t]{2}{*}{ G161.56-9.29 } & 1 & $04: 16: 15.7$ & $+37: 46: 18$ & 161.56 & -9.29 & $13.0(0.7)$ & $13.3(1.8)$ & $1.9(0.5)$ & $1.2(0.5)$ \\
\hline & 2 & $04: 16: 51.5$ & $+37: 45: 42$ & 161.65 & -9.22 & - & - & - & - \\
\hline G162.4-08.7A1 & 1 & $04: 21: 29.0$ & $+37: 34: 31$ & 162.44 & -8.70 & $12.1(0.3)$ & $12.1(0.7)$ & $2.0(0.2)$ & $5.3(1.1)$ \\
\hline G163.32-8.41 & 1 & $04: 25: 32.9$ & $+37: 09: 00$ & 163.32 & -8.41 & $11.7(0.4)$ & $11.4(1.7)$ & $2.1(0.5)$ & $5.7(3.0)$ \\
\hline G163.68-8.33 & 1 & $04: 27: 06.5$ & $+36: 56: 55$ & 163.68 & -8.33 & $11.9(0.4)$ & $11.2(1.2)$ & $2.3(0.4)$ & $3.7(1.5)$ \\
\hline G163.82-8.33 & 1 & $04: 27: 33.2$ & $+36: 50: 32$ & 163.82 & -8.33 & $11.9(0.6)$ & $11.0(1.9)$ & $2.3(0.7)$ & $3.5(2.2)$ \\
\hline G164.1-08.8A1 & 1 & $04: 26: 54.5$ & $+36: 14: 11$ & 164.18 & -8.84 & $11.9(0.6)$ & $10.7(1.6)$ & $2.5(0.6)$ & $2.8(1.6)$ \\
\hline G164.11-8.17 & 1 & 04:29:07.7 & $+36: 44: 35$ & 164.11 & -8.17 & $12.3(0.5)$ & $11.6(1.7)$ & $2.3(0.6)$ & $1.8(1.0)$ \\
\hline G164.26-8.39 & 1 & $04: 28: 50.0$ & $+36: 29: 15$ & 164.26 & -8.39 & $12.8(0.5)$ & $12.1(1.5)$ & $2.3(0.5)$ & $2.2(1.0)$ \\
\hline G165.1-07.5A1 & 1 & 04:34:50.0 & $+36: 23: 20$ & 165.16 & -7.57 & $12.4(0.4)$ & $11.9(1.1)$ & $2.2(0.4)$ & $2.7(0.9)$ \\
\hline G165.3-07.5A1 & 1 & $04: 35: 42.0$ & $+36: 16: 35$ & 165.36 & -7.51 & $12.2(0.3)$ & $11.6(0.8)$ & $2.2(0.2)$ & $3.3(0.8)$ \\
\hline G165.6-09.1A1 & 1 & 04:30:53.1 & $+34: 55: 24$ & 165.71 & -9.15 & $12.8(2.3)$ & - & - & $3.0(1.7)$ \\
\hline G167.2-15.3A1 & 1 & $04: 14: 28.6$ & $+29: 35: 25$ & 167.23 & -15.33 & $12.2(0.2)$ & $12.1(0.6)$ & $2.1(0.2)$ & $2.7(0.5)$ \\
\hline G168.1-16.3A1 & 1 & $04: 13: 43.3$ & $+28: 15: 15$ & 168.10 & -16.38 & $10.6(0.4)$ & $9.5(2.3)$ & $2.5(0.7)$ & $18.4(15.9)$ \\
\hline G169.1-01.1A1 & 1 & 18.6 & $+37: 20: 28$ & 169.15 & -1.15 & 13.6 & $17.1(1.4)$ & $1.5(0.2)$ & $2.1(0.5)$ \\
\hline G170.0-16.1A1 & 1 & $04: 20: 13.3$ & $+27: 06: 19$ & 170.00 & -16.13 & $12.3(2.1)$ & - & - & $1.6(1.0)$ \\
\hline \multirow[t]{2}{*}{ G170.1-16.0A1 } & 1 & $04: 20: 50.2$ & $+27: 03: 10$ & 170.14 & -16.07 & $11.4(0.7)$ & $11.3(2.9)$ & $2.0(0.8)$ & $5.2(4.3)$ \\
\hline & 2 & $04: 21: 17.5$ & $+26: 59: 27$ & 170.26 & -16.04 & 11.0 & $10.8(3.6)$ & $2.1(1.2)$ & $6.1)$ \\
\hline G170.8-18.3A1 & 1 & $04: 15: 23.5$ & $+25: 02: 02$ & 170.81 & -18.34 & 13.3 & - & - & $0.5)$ \\
\hline G170.9-15.8A1 & 1 & $04: 24: 14.0$ & $+26: 37: 19$ & 171.00 & -15.80 & $10.6(0.3)$ & $10.0(2.4)$ & $2.3(0.6)$ & $7.5(6.2)$ \\
\hline G171.14-17.57 & 1 & 04:18:50.1 & $+25: 19: 29$ & 171.14 & -17.57 & $11.3(0.2)$ & $9.8(0.7)$ & $2.6(0.3)$ & $8.6(2.7)$ \\
\hline G171.8-15.3A1 & 1 & $04: 28: 06.8$ & $+26: 22: 00$ & 171.80 & -15.32 & $11.8(1.0)$ & - & - & $3.5(1.3)$ \\
\hline G172.8-14.7A1 & 1 & & $+25: 59: 55$ & 172.84 & -14.74 & 12.2( & $12.0(1.4)$ & $2.1(0.4)$ & $2.8(1.2)$ \\
\hline G173.1-13.3A1 & 1 & $04: 38: 42.4$ & $+26: 41: 50$ & 173.14 & -13.32 & $12.6(1.7)$ & - & - & $1.8(0.9)$ \\
\hline G173.3-16.2A1 & 1 & $04: 29: 20.5$ & $+24: 37: 35$ & 173.35 & -16.26 & $12.0(0.6)$ & - & - & $6.9(1.3)$ \\
\hline G173.9-13.7A1 & 1 & 04:39:27.7 & $+25: 48: 25$ & 173.95 & -13.76 & $12.1(1.3)$ & - & - & $6.4(2.5)$ \\
\hline G174.0-15.8A1 & 1 & $04: 32: 44.7$ & $+24: 24: 09$ & 174.05 & -15.82 & $11.3(0$ & - & - & $8.4(2.1)$ \\
\hline G174.4-15.7A1 & 1 & $04: 33: 58.6$ & $+24: 10: 21$ & 174.42 & -15.76 & 12.5 & - & - & $1.7(1.5)$ \\
\hline G174.7-15.4A1 & 1 & $04: 35: 41.7$ & $+24: 09: 28$ & 174.70 & -15.47 & $11.3(0.2)$ & $10.6(0.7)$ & $2.3(0.2)$ & $12.0(3.3)$ \\
\hline G174.8-17.1A1 & 1 & $04: 30: 43.8$ & $+22: 55: 59$ & 174.90 & -17.13 & $11.8(0.4)$ & $11.1(0.9)$ & $2.3(0.4)$ & $3.0(1.0)$ \\
\hline $\mathrm{G} 175.2+01.2 \mathrm{~A} 2$ & 1 & $05: 38: 56.8$ & $+33: 41: 15$ & 175.21 & 1.29 & $11.7(0.7)$ & $12.5(1.4)$ & $1.8(0.4)$ & $1.5(0.6)$ \\
\hline G175.4-16.8A2 & 1 & & & & -16 & & - & - & \\
\hline G177.6-20.3A1 & 1 & 04:27:17.3 & $+18: 51: 43$ & 177.64 & -20.37 & $12.9(0.4)$ & $12.8(1.6)$ & $2.0(0.5)$ & $1.7(0.7)$ \\
\hline G181.8+00.3A1 & 1 & $05: 51: 12.6$ & $+27: 30: 15$ & 181.86 & 0.32 & $11.6(0.5)$ & $11.4(1.2)$ & $2.1(0.4)$ & $3.6(1.4)$ \\
\hline G201.2+00.4A1 & 1 & $06: 31: 33.1$ & $+10: 34: 06$ & 201.27 & 0.45 & $12.5(7.2)$ & - & - & $1.4(1.6)$ \\
\hline G201.83+2.83 & 1 & $06: 41: 13.0$ & $+11: 09: 56$ & 201.83 & 2.83 & $13.4(2.3)$ & - & - & - \\
\hline G202.00+2.65 & 1 & $06: 40: 53.2$ & $+10: 55: 56$ & 202.00 & 2.65 & $12.9(2.4)$ & - & - & $1.6(1.0)$ \\
\hline G202.54+2.46 & 1 & 06:41:10.8 & $+10: 22: 19$ & 202.54 & 2.46 & $14.1(0.8)$ & $15.0(1.8)$ & $1.8(0.4)$ & $2.8(1.0)$ \\
\hline G204.4-11.3A2 & 1 & $05: 55: 43.9$ & $+02: 12: 19$ & 204.49 & -11.33 & $11.1(1.9)$ & - & - & $2.6(1.5)$ \\
\hline G204.8-13.8A1 & 1 & $05: 47: 26.0$ & $+00: 42: 47$ & 204.83 & -13.86 & $13.2(1.8)$ & - & - & $5.7(2.6)$ \\
\hline G210.90-36.55 & 1 & 04:35:08.6 & $-14: 15: 27$ & 210.90 & -36.55 & $12.0(0.3)$ & $11.0(0.6)$ & $2.4(0.2)$ & $5.3(1.2)$ \\
\hline G215.44-16.38 & 1 & $05: 57: 01.7$ & $-09: 33: 14$ & 215.44 & -16.38 & $12.5(0.4)$ & $12.1(0.8)$ & $2.1(0.3)$ & $1.7(0.4)$ \\
\hline G216.76-2.58 & 1 & $06: 49: 13.6$ & $-04: 34: 59$ & 216.76 & -2.58 & $10.4(1.1)$ & - & - & $4.0(1.7)$ \\
\hline \multirow[t]{2}{*}{ G219.13-9.72 } & 1 & $06: 27: 28.1$ & $-09: 54: 36$ & 219.12 & -9.79 & $12.9(1.1)$ & $13.6(2.3)$ & $1.8(0.7)$ & $1.4(0.8)$ \\
\hline & 2 & $06: 27: 42.7$ & $-09: 53: 06$ & 219.13 & -9.72 & $13.0(1.6)$ & $14.1(4.3)$ & $1.7(1.1)$ & - \\
\hline G219.22-10.02 & 1 & $06: 26: 46.9$ & $-10: 05: 45$ & 219.22 & -10.02 & $12.0(2.7)$ & - & - & $1.6(1.2)$ \\
\hline G219.36-9.71 & 1 & 06:28:09.8 & $-10: 05: 11$ & 219.36 & -9.71 & $12.9(0.5)$ & $13.0(1.1)$ & $2.0(0.3)$ & $3.4(1.0)$ \\
\hline
\end{tabular}




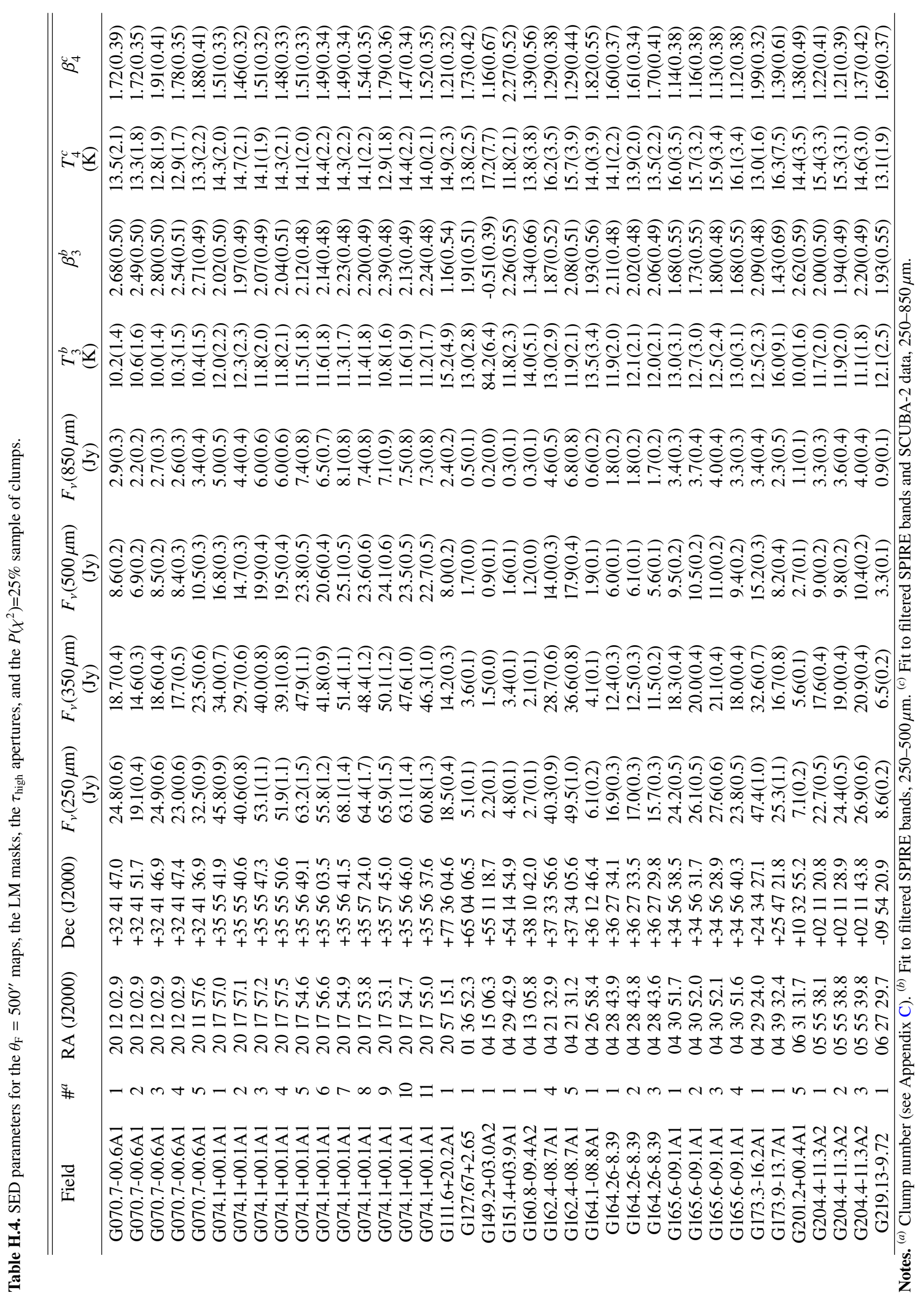

A71, page 38 of 38 